Supporting Information

\title{
Catalytic Asymmetric Hydrophosphination as a Valuable Tool to Access Di-Hydrophosphinated Curcumin and its Derivatives
}

\author{
Ronald Hong Xiang Teo, ${ }^{\mathrm{a}}$ Jeannie Xue Ting Lee,, , Wei Ren Tan, ${ }^{\mathrm{b}}$ Wen Qian Shum, ${ }^{\mathrm{a}}$ \\ Yongxin $\mathrm{Li}^{\mathrm{a}}{ }^{\mathrm{a}}$ Sumod A. Pullarkat, ${ }^{\mathrm{a}}$ Nguan Soon Tan ${ }^{\mathrm{b}, \mathrm{c}, *}$ and Pak-Hing Leung ${ }^{\mathrm{a}, *}$ \\ ${ }^{a}$ Division of Chemistry and Biological Chemistry, School of Physical and Mathematical Sciences, Nanyang \\ Technological University, Singapore 637371, Singapore, ${ }^{b}$ Lee Kong Chian School of Medicine, Nanyang \\ Technological University Singapore, 11 Mandalay Road, Singapore 308232, Singapore, ${ }^{c}$ School of Biological \\ Sciences, Nanyang Technological University, 60 Nanyang Drive, Singapore 637551, Singapore
}

Email: nstan@ntu.edu.sg; pakhing@ntu.edu.sg 


\section{TABLE OF CONTENTS}

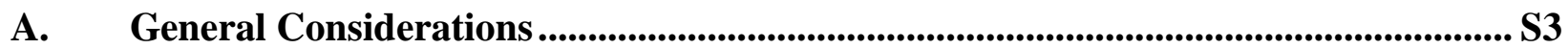

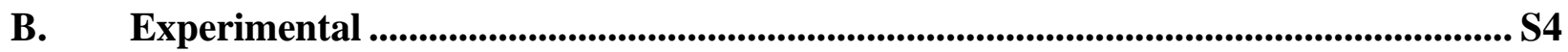

B1 Optimization of procedure for the catalytic asymmetric dihydrophosphination of

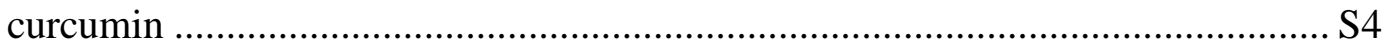

B2 General procedure for the catalytic asymmetric dihydrophosphination reaction. S6

B3 One-pot direct dihydrophosphination-complexation of diphosphines................ S12

C. NMR Spectroscopy Data .................................................................................................... S14

C1 Mono-hydrophosphinated curcumin sulfide mono-2aa ................................... S14

C2 $D i$-hydrophosphinated curcumin sulfide chiral-3aa..................................... S17

C3 Di-hydrophosphinated curcumin sulfide meso-3aa' ....................................... S20

C4 Di-hydrophosphinated bisdemethoxycurcumin sulfide chiral-3ba and meso-3ba'

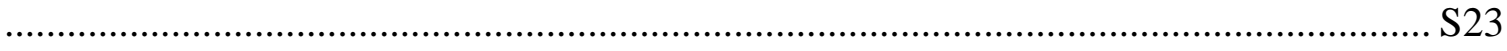

C5 Di-hydrophosphinated curcumin sulfide chiral-3ab ..................................... S25

C6 Di-hydrophosphinated curcumin sulfide meso-3ab' ...................................... S28

C7 Di-hydrophosphinated curcumin sulfide chiral-3ac and meso-3ac' .................. S31

C8 Di-hydrophosphinated curcumin sulfide chiral-3ad and meso-3ad' '................. S33

C9 Di-hydrophosphinated curcumin sulfide chiral-3ae ....................................... S35

C10 Di-hydrophosphinated curcumin sulfide mono-2ae and meso-3ae' ................... S38

C11 Di-hydrophosphinated curcumin Au(I) rac-4aa ............................................. S40

C12 Di-hydrophosphinated curcumin $\mathrm{Au}(\mathrm{I})$ meso-4aa' ............................................ S43

D. Diastereomeric Ratios and Enantiomeric Excess Data ..............................................S46

D1 Diastereomeric ratio of chiral-3aa and meso-3aa' ........................................... S46

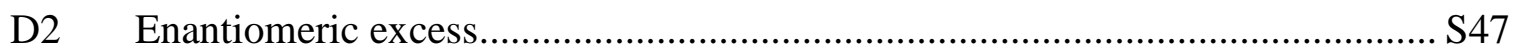

E. DETECTION OF CHARACTERISTIC PROTON SIGNAL OF ENOL IN DIHYDROPHOSPHINATED PRODUCT ................................................................... S61

E1 Evidence of Keto-Enol Equilibrium ………………………….................... S61

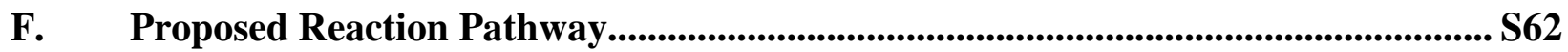

F1 Proposed Mechanistic Cycle ………………….......................................... S62

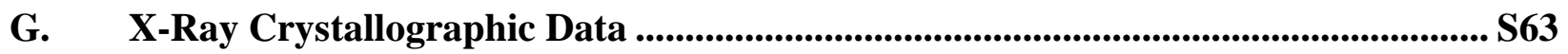

G1 Mono-hydrophosphinated curcumin sulfide mono-2aa .................................... S63

G2 Di-hydrophosphinated curcumin sulfide chiral-3aa........................................ S65

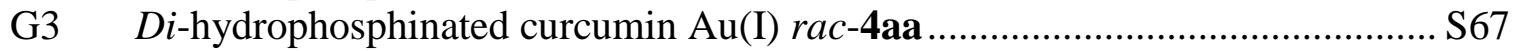

G4 Di-hydrophosphinated curcumin Au(I) meso-4aa' ............................................ S69 


\section{A GENERAL CONSIDERATIONS}

Reactions involving air- or moisture-sensitive compounds were carried out by means of conventional Schlenk techniques under a positive pressure of nitrogen gas. Unless stated otherwise, chemicals and solvents were used as received from commercial vendors without further purification.

Nuclear magnetic resonance (NMR) spectroscopy was recorded at Nanyang Technological University Division of Chemistry and Biological Chemistry Central Facilities Laboratory on the Bruker Avance 400 spectrometer at $400 \mathrm{MHz}$ for ${ }^{1} \mathrm{H}$ NMR, $161 \mathrm{MHz}$ for ${ }^{31} \mathrm{P}\left\{{ }^{1} \mathrm{H}\right\}$ and $100 \mathrm{MHz}$ for ${ }^{13} \mathrm{C}$ NMR. Chemical shifts $(\delta)$ are quoted in ppm and referenced to chemical shifts of residual solvent peaks for ${ }^{1} \mathrm{H}$ and ${ }^{13} \mathrm{C}$ NMR.

Enantiomeric excess (ee) was determined with an Agilent 1200 series high performance liquid chromatography (HPLC) system fitted with Daicel Chiralpak ${ }^{\circledR}$ I-series analytical columns (250 $\mathrm{mm}$ ), eluted with a solvent mixture of $n$-hexane and 2-propanol.

High resolution mass spectrometry (HRMS) via electrospray ionization (ESI) was performed on the Waters Q-Tof Premier spectrometer. Unless stated otherwise, acetonitrile was used as the solvent for the dissolution of compounds.

Optical rotation studies were measured on the Jasco P-1030 polarimeter in a $0.1 \mathrm{dm}$ polarimetry cell at the specified temperature using the D-line of sodium $(589 \mathrm{~nm})$ as the source of light. Unless stated otherwise, dichloromethane was used as the solvent for the dissolution of compounds. 


\section{B EXPERIMENTAL}

\section{B1 Optimization of Procedure for the Catalytic Asymmetric Dihydrophosphination of Curcumin}

Table S1. Optimization of Catalytic Asymmetric Dihydrophosphination of Curcumin.

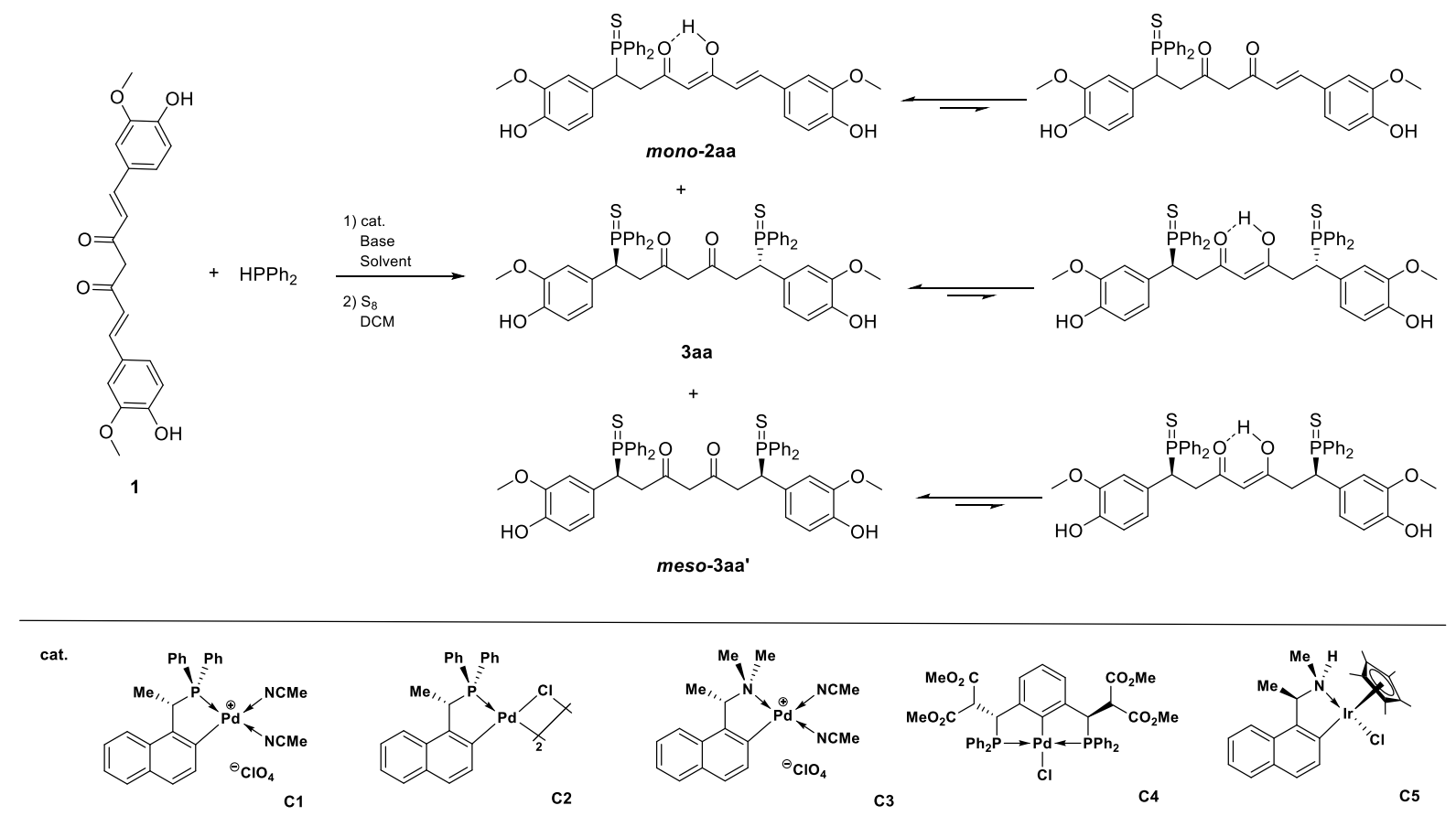

To a degassed solution $(7.5 \mathrm{~mL})$ of diphenylphosphine $(52.1 \mathrm{mg}, 0.28 \mathrm{mmol})$ was added catalyst. The mixture was subsequently cooled to the listed temperature prior to addition of curcumin $(51.6 \mathrm{mg}, 0.14 \mathrm{mmol})$ and base. The reaction mixture was then stirred at the same temperature for $120 \mathrm{~h} . \mathrm{S}_{8}(9.92 \mathrm{mg}, 0.31 \mathrm{mmol})$ was added to the crude mixture and stirred at ambient temperature for $2 \mathrm{~h}$. The solution was purified directly by column chromatography on silica gel with 199:1 dichloromethane/ $\mathrm{MeOH}$ as the eluent to provide the products.

\begin{tabular}{|c|c|c|c|c|c|c|c|c|}
\hline entry & catalyst & cat. loading & base & base loading & solvent & $\begin{array}{c}\text { conversion } \\
(\%)^{b}\end{array}$ & $e e(\%)^{c}$ & entry \\
\hline 1 & C1 & $2 \mathrm{~mol} \%$ & ${ }^{i} \mathrm{Pr}_{2} \mathrm{NH}$ & $20 \mathrm{~mol} \%$ & $\mathrm{MeOH}$ & 90 & 71 & 1 \\
\hline 2 & C1 & $2 \mathrm{~mol} \%$ & ${ }^{i} \mathrm{Pr}_{2} \mathrm{NH}$ & $20 \mathrm{~mol} \%$ & $\mathrm{EtOH}$ & 83 & 64 & 2 \\
\hline 3 & C1 & $2 \mathrm{~mol} \%$ & ${ }^{i} \mathrm{Pr}_{2} \mathrm{NH}$ & $20 \mathrm{~mol} \%$ & DCM & $38^{d}$ & n.d & 3 \\
\hline 4 & C1 & $2 \mathrm{~mol} \%$ & ${ }^{i} \mathrm{Pr}_{2} \mathrm{NH}$ & $20 \mathrm{~mol} \%$ & $\mathrm{MeCN}$ & $63^{d}$ & n.d & 4 \\
\hline 5 & C1 & $2 \mathrm{~mol} \%$ & ${ }^{i} \mathrm{Pr}_{2} \mathrm{NH}$ & $20 \mathrm{~mol} \%$ & Toluene & n.r & - & 5 \\
\hline 6 & C1 & $2 \mathrm{~mol} \%$ & ${ }^{i} \mathrm{Pr}_{2} \mathrm{NH}$ & $20 \mathrm{~mol} \%$ & THF & n.r & - & 6 \\
\hline 7 & C1 & $2 \mathrm{~mol} \%$ & $\mathrm{NEt}_{3}$ & $20 \mathrm{~mol} \%$ & $\mathrm{MeOH}$ & 80 & 74 & 7 \\
\hline 8 & C1 & $2 \mathrm{~mol} \%$ & $\mathrm{NaOAc}$ & $20 \mathrm{~mol} \%$ & $\mathrm{MeOH}$ & 88 & 71 & 8 \\
\hline 9 & C1 & $2 \mathrm{~mol} \%$ & DBU & $20 \mathrm{~mol} \%$ & $\mathrm{MeOH}$ & 77 & 30 & 9 \\
\hline 10 & C1 & $2 \mathrm{~mol} \%$ & $\mathrm{Cs} 2 \mathrm{CO} 3$ & $20 \mathrm{~mol} \%$ & $\mathrm{MeOH}$ & 64 & 65 & 10 \\
\hline 11 & C1 & $2 \mathrm{~mol} \%$ & CsF & $20 \mathrm{~mol} \%$ & $\mathrm{MeOH}$ & 76 & 52 & 11 \\
\hline 12 & $\mathrm{C1}$ & $2 \mathrm{~mol} \%$ & DIPEA & $20 \mathrm{~mol} \%$ & $\mathrm{MeOH}$ & 81 & 66 & 12 \\
\hline 13 & $\mathrm{C1}$ & $1 \mathrm{~mol} \%$ & $\mathrm{NEt}_{3}$ & $20 \mathrm{~mol} \%$ & $\mathrm{MeOH}$ & 57 & 41 & 13 \\
\hline 14 & C1 & $4 \mathrm{~mol} \%$ & $\mathrm{NEt}_{3}$ & $20 \mathrm{~mol} \%$ & $\mathrm{MeOH}$ & 85 & 79 & 14 \\
\hline 15 & C1 & $2.5 \mathrm{~mol} \%$ & $\mathrm{NEt}_{3}$ & $20 \mathrm{~mol} \%$ & $\mathrm{MeOH}$ & 86 & 80 & 15 \\
\hline 16 & C1 & $2.5 \mathrm{~mol} \%$ & $\mathrm{NEt}_{3}$ & $50 \mathrm{~mol} \%$ & $\mathrm{MeOH}$ & 80 & 66 & 16 \\
\hline 17 & $\mathrm{C1}$ & $2.5 \mathrm{~mol} \%$ & $\mathrm{NEt}_{3}$ & $100 \mathrm{~mol} \%$ & $\mathrm{MeOH}$ & 79 & 64 & 17 \\
\hline
\end{tabular}




$\begin{array}{lllllllll}18 & \mathbf{C 2} & 2.5 \mathrm{~mol} \% & \mathrm{NEt}_{3} & 20 \mathrm{~mol} \% & \mathrm{MeOH} & 83 & 52 & 18 \\ 19 & \mathbf{C 3} & 2.5 \mathrm{~mol} \% & \mathrm{NEt}_{3} & 20 \mathrm{~mol} \% & \mathrm{MeOH} & 85 & 25 & 19 \\ 20 & \mathbf{C 4} & 2.5 \mathrm{~mol} \% & \mathrm{NEt}_{3} & 20 \mathrm{~mol} \% & \mathrm{MeOH} & 78 & 34 & 20 \\ 21 & \mathbf{C 5} & 2.5 \mathrm{~mol} \% & \mathrm{NEt}_{3} & 20 \mathrm{~mol} \% & \mathrm{MeOH} & 75 & 5 & 21 \\ 22^{e} & \mathbf{C 1} & 2.5 \mathrm{~mol} \% & \mathrm{NEt}_{3} & 20 \mathrm{~mol} \% & \mathrm{MeOH} & 100 & 26 & 22^{e} \\ 23^{f} & \mathbf{C 1} & 2.5 \mathrm{~mol} \% & \mathrm{NEt}_{3} & 20 \mathrm{~mol} \% & \mathrm{MeOH} & 83 & 93 & 23^{f}\end{array}$

${ }^{a}$ Reactions were carried out with curcumin $(0.14 \mathrm{mmol})$, diphenylphosphine $(0.28 \mathrm{mmol})$, catalyst and base in the listed solvent $(7.5 \mathrm{~mL})$ at room temperature for $120 \mathrm{~h} .{ }^{b}$ Conversion was based on percentage of diphenylphosphine reacted. ${ }^{c}$ Enantiomeric excess of chiral diphosphine determined by chiral HPLC. ${ }^{d}$ Reaction furnished mostly monophosphine-2aa. ${ }^{e}$ Reaction conducted at $50{ }^{\circ} \mathrm{C}$ for $60 \mathrm{~h} .{ }^{f}$ Reaction conducted at $0{ }^{\circ} \mathrm{C}$ for 120 h. 


\section{B2 General Procedure for the Catalytic Asymmetric Dihydrophosphination Reaction}

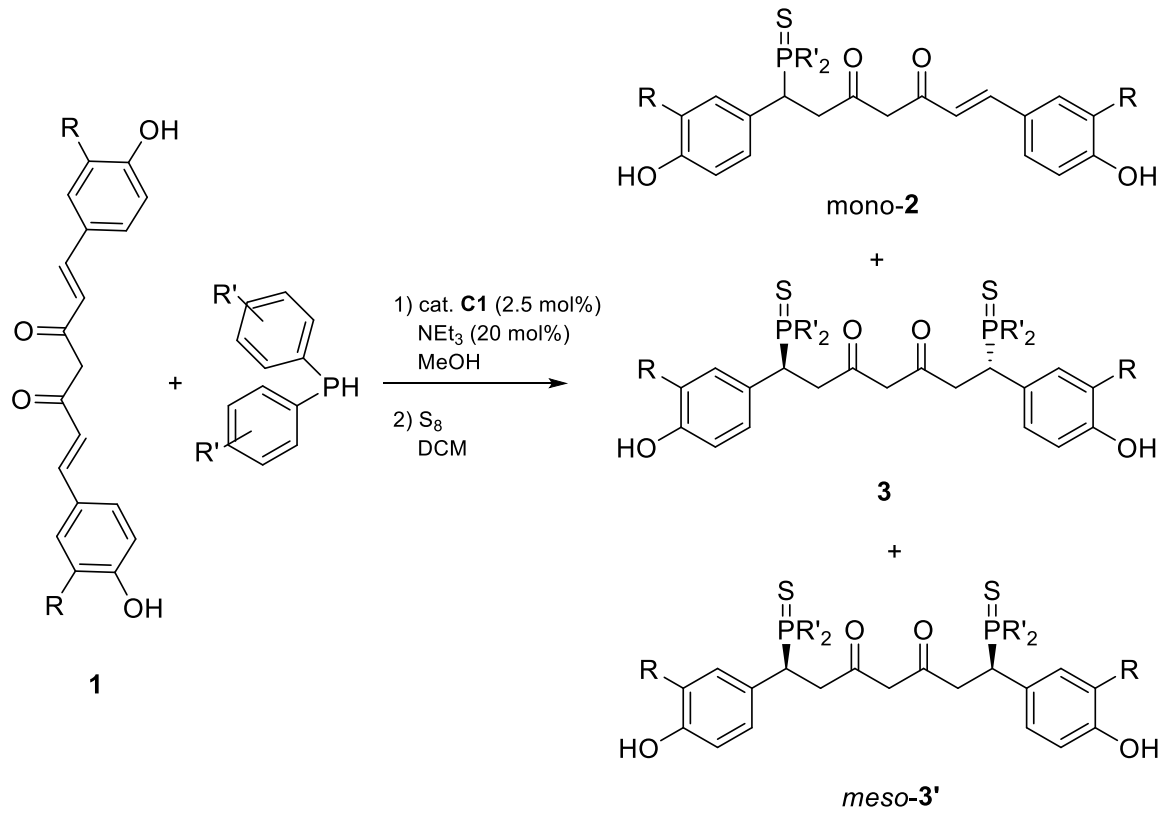

Scheme S1. Catalytic Asymmetric Dihydrophosphination.

Catalyst C1 (2.51 mg, $2.5 \mathrm{~mol} \%)$ was added to a solution of secondary phosphines (0.16 mmol) in dry and degassed $\mathrm{MeOH}(7.5 \mathrm{~mL})$. The mixture was subsequently cooled to $0{ }^{\circ} \mathrm{C}$ before the addition of substrate $1(0.08 \mathrm{mmol})$ and $\mathrm{NEt}_{3}(4.46 \mathrm{mg}, 20 \mathrm{~mol} \%)$. The reaction mixture was then stirred for $120 \mathrm{~h} . \mathrm{S}_{8}(5.76 \mathrm{mg}, 0.18 \mathrm{mmol})$ was added to the crude mixture and stirred until the reaction completed. The solution was purified directly by preparatory thin layer chromatography (PTLC) to provide the products.

\section{Secondary Phosphine: Diphenylphosphine}<smiles>COc1cc(/C=C/C(=O)CC(=O)CC(c2ccc(O)c(OC)c2)P(=S)=S)ccc1O</smiles>

Figure S1. Structure of 2aa

Yellow Solid; Eluent system (PTLC): DCM/MeOH (199:1); Yield: 3.75 mg (4\%); ${ }^{1} \mathrm{H}$ NMR (400 $\left.\mathrm{MHz}, \mathrm{CD}_{2} \mathrm{Cl}_{2}\right): \delta 2.90\left(\mathrm{ddd}, 1 \mathrm{H}, J_{\mathrm{HH}}=16.2 \mathrm{~Hz}, J_{\mathrm{HP}}=10.7 \mathrm{~Hz}, J_{\mathrm{HH}}=3.1 \mathrm{~Hz}, \mathrm{PCHCHHCO}\right)$, $3.24\left(\mathrm{ddd}, 1 \mathrm{H}, J_{\mathrm{HH}}=16.1 \mathrm{~Hz}, J_{\mathrm{HH}}=11.0 \mathrm{~Hz}, J_{\mathrm{HP}}=6.9 \mathrm{~Hz}, \mathrm{PCHCH}_{2}\right), 3.68\left(\mathrm{~s}, 3 \mathrm{H}, \mathrm{CH}_{3} \mathrm{OAr}\right)$, $3.91\left(\mathrm{~s}, 3 \mathrm{H}, \mathrm{CH}_{3} \mathrm{OAr}\right), 4.56\left(\mathrm{ddd}, 1 \mathrm{H}, J_{\mathrm{HH}}=10.4 \mathrm{~Hz}, J_{\mathrm{HP}}=10.2 \mathrm{~Hz}, J_{\mathrm{HH}}=2.7 \mathrm{~Hz}, \mathrm{PCHCHHCO}\right)$, $5.50(\mathrm{~s}, 1 \mathrm{H}, \mathrm{OH}), 5.51(\mathrm{~s}, 1 \mathrm{H}, \mathrm{OH}), 5.93(\mathrm{~s}, 1 \mathrm{H}, \mathrm{COCHCO}), 6.23\left(\mathrm{~d}, 1 \mathrm{H}, J_{\mathrm{HH}}=15.8 \mathrm{~Hz}\right.$, $\mathrm{COCHCH}), 6.63-6.88(\mathrm{~m}, 4 \mathrm{H}, \mathrm{ArH}), 7.02-7.04(\mathrm{~m}, 2 \mathrm{H}, \mathrm{ArH}), 7.26-7.38(\mathrm{~m}, 3 \mathrm{H}, \mathrm{ArH}), 7.48(\mathrm{~d}$, $\left.1 \mathrm{H}, J_{\mathrm{HH}}=15.8 \mathrm{~Hz}, \mathrm{COCHCH}\right), 7.53-7.58(\mathrm{~m}, 5 \mathrm{H}, \operatorname{ArH}), 8.13-8.18(\mathrm{~m}, 2 \mathrm{H}, \operatorname{ArH}), 15.02(\mathrm{bs}, 1 \mathrm{H}$, $\mathrm{OHO}) ;{ }^{13} \mathrm{C} \mathrm{NMR}\left(100 \mathrm{MHz}, \mathrm{CD}_{2} \mathrm{Cl}_{2}\right): 41.16(\mathrm{~s}), 42.08(\mathrm{~s}), 42.01(\mathrm{~s}), 56.38$ (d, $\left.J_{\mathrm{CP}}=11.0 \mathrm{~Hz}\right)$, 
$101.53(\mathrm{~s}), 109.84(\mathrm{~s}), 113.09\left(\mathrm{~d}, J_{\mathrm{CP}}=4.7 \mathrm{~Hz}\right), 113.86\left(\mathrm{~d}, J_{\mathrm{CP}}=3.4 \mathrm{~Hz}\right), 114.99(\mathrm{~s}), 120.09(\mathrm{~s})$, $123.19(\mathrm{~s}), 123.24(\mathrm{~s}), 126.62\left(\mathrm{~d}, J_{\mathrm{CP}}=3.7 \mathrm{~Hz}\right), 127.98(\mathrm{~s}), 128.37\left(\mathrm{~d}, J_{\mathrm{CP}}=12.0 \mathrm{~Hz}\right), 129.23(\mathrm{~d}$, $\left.J_{\mathrm{CP}}=11.5 \mathrm{~Hz}\right), 131.63\left(\mathrm{~d}, J_{\mathrm{CP}}=2.4 \mathrm{~Hz}\right), 132.02\left(\mathrm{~d}, J_{\mathrm{CP}}=9.5 \mathrm{~Hz}\right), 132.31\left(\mathrm{~d}, J_{\mathrm{CP}}=9.3 \mathrm{~Hz}\right)$, $140.47(\mathrm{~s}), 145.50$ (d, $\left.J_{\mathrm{CP}}=3.0 \mathrm{~Hz}\right), 146.32$ (s), 146.34 (s) 147.38 (s), 148.35 (s), 153.04 (s), 156.85 (s), 197.92 (d, $\left.J_{\mathrm{CP}}=15.4 \mathrm{~Hz}\right) ;{ }^{31} \mathrm{P}\left\{{ }^{1} \mathrm{H}\right\} \mathrm{NMR}\left(161 \mathrm{MHz}, \mathrm{CD}_{2} \mathrm{Cl}_{2}\right): \delta 50.82$; HRMS (ESI) $\mathrm{m} / \mathrm{z}$ [positive mode] calcd. for $\mathrm{C}_{33} \mathrm{H}_{32} \mathrm{O}_{6} \mathrm{PS} 587.1657$, found 587.1657.

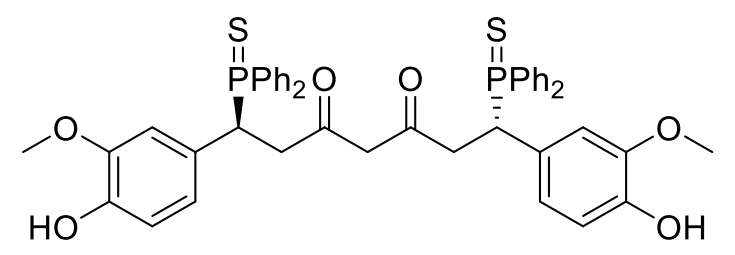

Figure S2. Structure of chiral-3aa

White Solid; Eluent system (PTLC): DCM/MeOH (199:1); Yield: 48.9 mg (38\%); ${ }^{1} \mathrm{H}$ NMR (400 $\mathrm{MHz}, \mathrm{CD}_{2} \mathrm{Cl}_{2}$ ): $\delta 2.67\left(\mathrm{ddd}, 1 \mathrm{H}, J_{\mathrm{HH}}=15.7 \mathrm{~Hz}, J_{\mathrm{HP}}=10.0 \mathrm{~Hz}, J_{\mathrm{HH}}=3.2 \mathrm{~Hz}, \mathrm{PCHCHHCO}\right.$ ), $2.95\left(\mathrm{ddd}, 1 \mathrm{H}, J_{\mathrm{HH}}=15.8 \mathrm{~Hz}, J_{\mathrm{HH}}=11.2 \mathrm{~Hz}, J_{\mathrm{HP}}=7.1 \mathrm{~Hz}, \mathrm{PCHCH} \mathrm{H}_{2} \mathrm{CO}\right), 3.65\left(\mathrm{~s}, 6 \mathrm{H}, \mathrm{CH}_{3} \mathrm{OAr}\right)$, $4.31\left(\mathrm{ddd}, 1 \mathrm{H}, J_{\mathrm{HH}}=10.6 \mathrm{~Hz}, J_{\mathrm{HP}}=10.6 \mathrm{~Hz}, J_{\mathrm{HH}}=3.1 \mathrm{~Hz}, \mathrm{PCHCHHCO}\right), 5.53(\mathrm{~s}, 2 \mathrm{H}, \mathrm{OH})$, 6.55-6.61 (m, 4H, ArH), 6.73 (s, 2H, $\left.\mathrm{COCH}_{2} \mathrm{CO}\right), 7.23-7.55(\mathrm{~m}, 18 \mathrm{H}, \mathrm{ArH}), 8.03-8.08(\mathrm{~m}, 4 \mathrm{H}$ $\mathrm{Ar} H) ;{ }^{13} \mathrm{C}$ NMR (100 MHz, $\mathrm{CD}_{2} \mathrm{Cl}_{2}$ ): $\delta 39.05$ (d, $\left.J=3.9 \mathrm{~Hz}\right), 42.35$ (s), 42.87 (s), 56.03 (s), $102.12(\mathrm{~s}), 112.82(\mathrm{~d}, J=4.7 \mathrm{~Hz}), 113.80(\mathrm{~d}, J=2.3 \mathrm{~Hz}), 123.22(\mathrm{~d}, J=6.1 \mathrm{~Hz}), 126.08(\mathrm{~d}, J=$ $4.6 \mathrm{~Hz}), 128.35(\mathrm{~d}, J=12.0 \mathrm{~Hz}), 129.18(\mathrm{~d}, J=11.5 \mathrm{~Hz}), 130.88(\mathrm{~s}), 131.35$ (s), $131.64(\mathrm{~s})$, $131.94(\mathrm{~d}, J=9.8 \mathrm{~Hz}), 132.17(\mathrm{~d}, J=9.1 \mathrm{~Hz}), 145.51(\mathrm{~d}, J=3.1 \mathrm{~Hz}), 146.31(\mathrm{~d}, J=2.5 \mathrm{~Hz})$ $190.84(\mathrm{~d}, J=15.7 \mathrm{~Hz}) ;{ }^{31} \mathrm{P}\left\{{ }^{1} \mathrm{H}\right\} \mathrm{NMR}\left(161 \mathrm{MHz}, \mathrm{CD}_{2} \mathrm{Cl}_{2}\right): \delta 50.55$; HRMS (ESI) m/z [positive mode] calcd. for $\mathrm{C}_{45} \mathrm{H}_{43} \mathrm{O}_{6} \mathrm{P}_{2} \mathrm{~S}_{2}$ 805.1976, found 805.1976.

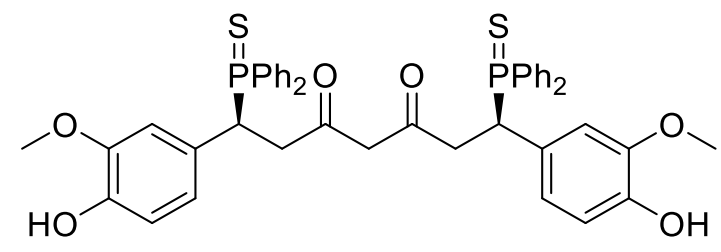

Figure S3. Structure of meso-3aa'

White Solid; Eluent system (PTLC): DCM/MeOH (199:1); Yield: $30.9 \mathrm{mg}(24 \%) ;{ }^{1} \mathrm{H}$ NMR (400 $\left.\mathrm{MHz}, \mathrm{CD}_{2} \mathrm{Cl}_{2}\right): \delta 2.69\left(\mathrm{ddd}, 1 \mathrm{H}, J_{\mathrm{HH}}=15.8 \mathrm{~Hz}, J_{\mathrm{HP}}=10.0 \mathrm{~Hz}, J_{\mathrm{HH}}=3.5 \mathrm{~Hz}, \mathrm{PCHCHHCO}\right.$ ), $2.92\left(\mathrm{ddd}, 1 \mathrm{H}, J_{\mathrm{HH}}=15.8 \mathrm{~Hz}, J_{\mathrm{HH}}=10.8 \mathrm{~Hz}, J_{\mathrm{HP}}=7.7 \mathrm{~Hz}, \mathrm{PCHCH} \mathrm{HO}_{2} \mathrm{CO}, 3.61\left(\mathrm{~s}, 6 \mathrm{H}, \mathrm{CH}_{3} \mathrm{OAr}\right)\right.$, $4.31\left(\mathrm{ddd}, 1 \mathrm{H}, J_{\mathrm{HH}}=10.5 \mathrm{~Hz}, J_{\mathrm{HP}}=10.5 \mathrm{~Hz}, J_{\mathrm{HH}}=3.4 \mathrm{~Hz}, \mathrm{PCHCHHCO}\right), 5.59(\mathrm{~s}, 2 \mathrm{H}, \mathrm{OH})$, 6.52-6.58 (m, 4H, $\mathrm{ArH}), 6.68\left(\mathrm{~s}, 2 \mathrm{H}, \mathrm{COCH}_{2} \mathrm{CO}\right), 7.23-7.37(\mathrm{~m}, 7 \mathrm{H}, \mathrm{ArH}), 7.46-7.56(\mathrm{~m}, 11 \mathrm{H}$, $\mathrm{ArH})$ 8.05-8.10 (m, 4H ArH); ${ }^{13} \mathrm{C}$ NMR (100 MHz, $\left.\mathrm{CD}_{2} \mathrm{Cl}_{2}\right): \delta 39.08$ (d, J=3.5 Hz), 42.29 (s). 42.81 (s), 56.22 (s), 102.22 (s), 112.71 (d, $J=4.7 \mathrm{~Hz}), 113.89$ (d, $J=2.7 \mathrm{~Hz}), 123.08$ (d, $J=6.1$ $\mathrm{Hz}), 126.10$ (d, $J=4.6 \mathrm{~Hz}), 128.35$ (d, $J=11.7 \mathrm{~Hz}), 129.16$ (d, $J=11.5 \mathrm{~Hz} 131.00$ (s), 131.35 (s), $131.63(\mathrm{~d}, J=3.2 \mathrm{~Hz}), 131.95(\mathrm{~d}, J=9.9 \mathrm{~Hz}), 132.23(\mathrm{~d}, J=9.4 \mathrm{~Hz}), 146.44(\mathrm{~d}, J=3.0 \mathrm{~Hz})$, $146.32(\mathrm{~d}, J=2.4 \mathrm{~Hz}), 190.80(\mathrm{~d}, J=15.4 \mathrm{~Hz}) ;{ }^{31} \mathrm{P}\left\{{ }^{1} \mathrm{H}\right\}$ NMR $\left(161 \mathrm{MHz}, \mathrm{CD}_{2} \mathrm{Cl}_{2}\right): \delta 50.52$; HRMS (ESI) m/z [positive mode] calcd. for $\mathrm{C}_{45} \mathrm{H}_{43} \mathrm{O}_{6} \mathrm{P}_{2} \mathrm{~S}_{2}$ 805.1976, found 805.1976. 


\section{Olefinic Substrate: bisdimethoxycurcumin}<smiles>O=C(CC(=O)c1ccccc1)CC(CC(=O)CC(c1ccc(O)cc1)P(=S)=S)P(=S)=S</smiles>

Figure S4. Structure of 3ba/3ba' (reported as an inseparable diastereomeric mixture)

White Solid; Eluent system (PTLC): Chloroform/MeOH (400:1) (provides pure chiral-3ba / meso-3ba' mixture); ${ }^{1} \mathrm{H} \mathrm{NMR}\left(400 \mathrm{MHz}, \mathrm{CD}_{2} \mathrm{Cl}_{2}\right): \delta 1.66$ (bs, $\left.-\mathrm{OH}\right), 2.57-2.71$ (m, -CHH-), 2.86-2.97 (m, -CHH-), 3.16-3.25 (m, -CHH-), 4.28-4.43 (m, -CHH-), 6.48-8.08 (m, ArH);

${ }^{31} \mathrm{P}\left\{{ }^{1} \mathrm{H}\right\}$ NMR $\left(161 \mathrm{MHz}, \mathrm{CD}_{2} \mathrm{Cl}_{2}\right): \delta 50.18,50.46$; HRMS (ESI) $\mathrm{m} / z$ [positive mode] calcd. for $\mathrm{C}_{43} \mathrm{H}_{39} \mathrm{O}_{4} \mathrm{P}_{2} \mathrm{~S}_{2} 745.1765$, found 745.1765.

\section{Secondary Phosphine: bis(4-methoxyphenyl)phosphine}<smiles>[R]Oc1ccc([Z]([H])(C)c2ccc(O)c(OC)c2)cc1</smiles>

Figure S5. Structure of 3ab

White Solid; Eluent system (PTLC): DCM/MeOH (199:1); Yield: 39.6 mg (41\%); ${ }^{1} \mathrm{H}$ NMR (400 $\left.\mathrm{MHz}, \mathrm{CD}_{2} \mathrm{Cl}_{2}\right): \delta 2.62\left(\mathrm{ddd}, 2 \mathrm{H}, J_{\mathrm{HH}}=15.7 \mathrm{~Hz}, J_{\mathrm{HP}}=9.8 \mathrm{~Hz}, J_{\mathrm{HH}}=3.1 \mathrm{~Hz}, \mathrm{PCHCHHCO}\right), 2.90$ $\left(\mathrm{ddd}, 2 \mathrm{H}, J_{\mathrm{HH}}=15.7 \mathrm{~Hz}, J_{\mathrm{HH}}=11.4 \mathrm{~Hz}, J_{\mathrm{HP}}=6.7 \mathrm{~Hz}, \mathrm{PCHCH} \mathrm{HO}_{2} \mathrm{CO}, 3.68\left(\mathrm{~s}, 6 \mathrm{H}, \mathrm{CH}_{3} \mathrm{OAr}\right), 3.74\right.$ $\left(\mathrm{s}, 6 \mathrm{H}, \mathrm{CH}_{3} \mathrm{OAr}\right), 3.84\left(\mathrm{~s}, 6 \mathrm{H}, \mathrm{CH}_{3} \mathrm{OAr}\right), 4.17\left(\mathrm{ddd}, 2 \mathrm{H}, J_{\mathrm{HH}}=10.8 \mathrm{~Hz}, J_{\mathrm{HP}}=10.6 \mathrm{~Hz}, J_{\mathrm{HH}}=3.0\right.$ $\mathrm{Hz}, \mathrm{PCHCHHCO}), 5.54(\mathrm{~s}, 2 \mathrm{H}, \mathrm{OH}), 6.52-6.55(\mathrm{~m}, 2 \mathrm{H}, \mathrm{ArH}), 6.59\left(\mathrm{~s}, 1 \mathrm{H}, \mathrm{COCH}_{2} \mathrm{CO}\right), 6.61(\mathrm{~s}$, $1 \mathrm{H}, \mathrm{COCH}_{2} \mathrm{CO}$ ), 6.74-6.77 (m, 7H, $\left.\mathrm{ArH}\right), 7.00-7.02$ (m, 4H ArH), 7.35-7.40 (m, 5H ArH), 7.927.97 (m, 4H ArH); ${ }^{13} \mathrm{C}$ NMR (100 MHz, $\left.\mathrm{CD}_{2} \mathrm{Cl}_{2}\right): \delta 39.07\left(\mathrm{~d}, J_{\mathrm{CP}}=3.3 \mathrm{~Hz}\right), 43.10(\mathrm{~s}), 43.63(\mathrm{~s})$, $55.72(\mathrm{~s}), 55.88(\mathrm{~s}), 56.30(\mathrm{~s}), 102.08(\mathrm{~s}), 112.80\left(\mathrm{~d}, J_{\mathrm{CP}}=4.3\right), 113.80\left(\mathrm{~d}, J_{\mathrm{CP}}=13.0\right), 114.67(\mathrm{~d}$, $\left.J_{\mathrm{CP}}=12.5\right), 121.84(\mathrm{~s}), 122.68\left(\mathrm{~d}, J_{\mathrm{CP}}=3.9\right), 123.24\left(\mathrm{~d}, J_{\mathrm{CP}}=6.1\right), 126.44\left(\mathrm{~d}, J_{\mathrm{CP}}=4.8\right), 133.77$ $\left(\mathrm{d}, J_{\mathrm{CP}}=11.2\right), 133.99\left(\mathrm{~d}, J_{\mathrm{CP}}=10.4\right), 145.43\left(\mathrm{~d}, J_{\mathrm{CP}}=3.0\right), 146.31\left(\mathrm{~d}, J_{\mathrm{CP}}=2.1\right), 162.43\left(\mathrm{~d}, J_{\mathrm{CP}}\right.$ $=2.4), 162.88\left(\mathrm{~d}, J_{\mathrm{CP}}=2.4\right), 191.17\left(\mathrm{~d}, J_{\mathrm{CP}}=16.2\right) ;{ }^{31} \mathrm{P}\left\{{ }^{1} \mathrm{H}\right\} \mathrm{NMR}\left(161 \mathrm{MHz}, \mathrm{CD}_{2} \mathrm{Cl}_{2}\right): \delta 49.03$; HRMS (ESI) m/z [positive mode] calcd. for $\mathrm{C}_{49} \mathrm{H}_{51} \mathrm{O}_{10} \mathrm{P}_{2} \mathrm{~S}_{2}$ 925.2399, found 925.2399. 


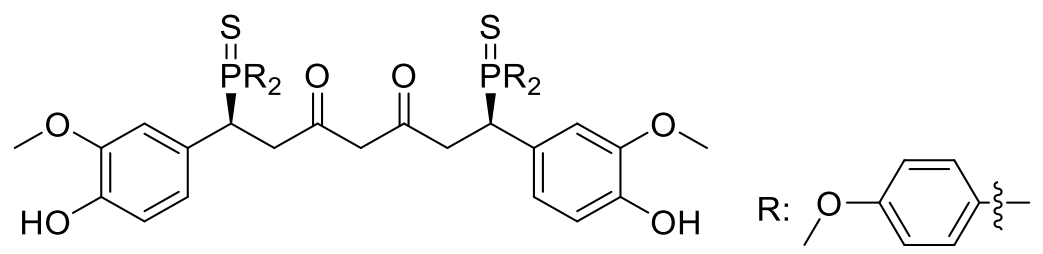

Figure S6. Structure of 3ab'

White Solid; Eluent system (PTLC): DCM/MeOH (199:1); Yield: $21.2 \mathrm{mg}$ (22\%); ${ }^{1} \mathrm{H}$ NMR (400 $\left.\mathrm{MHz}, \mathrm{CD}_{2} \mathrm{Cl}_{2}\right): \delta 2.66\left(\mathrm{ddd}, 2 \mathrm{H}, J_{\mathrm{HH}}=15.7 \mathrm{~Hz}, J_{\mathrm{HP}}=10.1 \mathrm{~Hz}, J_{\mathrm{HH}}=3.4 \mathrm{~Hz}, \mathrm{PCHCHHCO}\right.$ ), $2.87\left(\mathrm{ddd}, 2 \mathrm{H}, J_{\mathrm{HH}}=15.8 \mathrm{~Hz}, J_{\mathrm{HH}}=11.0 \mathrm{~Hz}, J_{\mathrm{HP}}=7.6 \mathrm{~Hz}, \mathrm{PCHCH}_{2} \mathrm{CO}\right), 3.64\left(\mathrm{~s}, 6 \mathrm{H}, \mathrm{CH}_{3} \mathrm{OAr}\right)$, $3.74\left(\mathrm{~s}, 6 \mathrm{H}, \mathrm{CH}_{3} \mathrm{OAr}\right), 3.84\left(\mathrm{~s}, 6 \mathrm{H}, \mathrm{CH}_{3} \mathrm{OAr}\right), 4.17\left(\mathrm{ddd}, 2 \mathrm{H}, J_{\mathrm{HH}}=10.7 \mathrm{~Hz}, J_{\mathrm{HP}}=10.6 \mathrm{~Hz}, J_{\mathrm{HH}}=\right.$ $3.0 \mathrm{~Hz}, \mathrm{PCHCHHCO}), 5.57$ (s, 2H, OH), 6.49-6.52 (m, $2 \mathrm{H}, \mathrm{Ar} H), 6.59$ (s, $\left.1 \mathrm{H}, \mathrm{COCH}_{2} \mathrm{CO}\right), 6.61$ (s, $1 \mathrm{H}, \mathrm{COCH}_{2} \mathrm{CO}$ ), 6.74-6.77 (m, 7H, $\mathrm{ArH}$ ), 7.00-7.02 (m, 4H $\left.\mathrm{ArH}\right), 7.35-7.40$ (m, 5H ArH), 7.92-7.97 (m, 4H ArH); ${ }^{13} \mathrm{C}$ NMR $\left(100 \mathrm{MHz}, \mathrm{CD}_{2} \mathrm{Cl}_{2}\right): \delta 39.13\left(\mathrm{~d}, J_{\mathrm{CP}}=4.1 \mathrm{~Hz}\right), 43.02(\mathrm{~s})$, $43.55(\mathrm{~s}), 55.73(\mathrm{~s}), 55.88(\mathrm{~s}), 56.23(\mathrm{~s}), 102.14(\mathrm{~s}), 112.70\left(\mathrm{~d}, J_{\mathrm{CP}}=4.6\right), 113.80\left(\mathrm{~d}, J_{\mathrm{CP}}=13.0\right)$, $114.62\left(\mathrm{~d}, J_{\mathrm{CP}}=12.6\right), 121.91(\mathrm{~s}), 122.71\left(\mathrm{~d}, J_{\mathrm{CP}}=2.2\right), 123.11\left(\mathrm{~d}, J_{\mathrm{CP}}=6.1\right), 126.50\left(\mathrm{~d}, J_{\mathrm{CP}}=\right.$ $4.6), 133.78\left(\mathrm{~d}, J_{\mathrm{CP}}=11.0\right), 134.60\left(\mathrm{~d}, J_{\mathrm{CP}}=10.4\right), 145.37\left(\mathrm{~d}, J_{\mathrm{CP}}=3.1\right), 146.32\left(\mathrm{~d}, J_{\mathrm{CP}}=2.5\right)$, $162.42\left(\mathrm{~d}, J_{\mathrm{CP}}=2.6\right), 162.87\left(\mathrm{~d}, J_{\mathrm{CP}}=3.1\right), 191.13\left(\mathrm{~d}, J_{\mathrm{CP}}=15.6\right) ;{ }^{31} \mathrm{P}\left\{{ }^{1} \mathrm{H}\right\}$ NMR $(161 \mathrm{MHz}$, $\mathrm{CD}_{2} \mathrm{Cl}_{2}$ ): $\delta$ 49.04; HRMS (ESI) $\mathrm{m} / \mathrm{z}$ [positive mode] calcd. for $\mathrm{C}_{49} \mathrm{H}_{51} \mathrm{O}_{10} \mathrm{P}_{2} \mathrm{~S}_{2}$ 925.2399, found 925.2413.

\section{Secondary Phosphine: bis(2-methoxyphenyl)phosphine}<smiles>[R]Oc1ccc(C(CC(=O)CC(=O)CC([R6])c2ccc(O)c(OC)c2)PSC)cc1OC</smiles>

Figure S7. Structure of 3ac/3ac' (reported as an inseparable diastereomeric mixture)

White Solid; Eluent system (PTLC): DCM/MeOH (199:1) (provides pure chiral-3ac / meso-3ac' mixture); ${ }^{1} \mathrm{H}$ NMR (400 MHz, $\mathrm{CD}_{2} \mathrm{Cl}_{2}$ ): $\delta 3.12-3.19$ (m, - $\mathrm{CH}-$ ), 3.53-3.60 (m, - $\mathrm{CH}-$ ), 3.63 (s, $\left.\mathrm{CH}_{3^{-}}\right), 3.65-3.69\left(\left(\mathrm{~m},-\mathrm{CH}_{2^{-}}\right), 3.67\left(\mathrm{~s},-\mathrm{CH}_{3^{-}}\right), 3.69\left(\mathrm{~s},-\mathrm{CH}_{3^{-}}\right), 3.74\left(\mathrm{~s},-\mathrm{CH}_{3^{-}}\right), 4.28-4.43(\mathrm{~m},-\right.$ $\mathrm{CH}_{2}-$ ), 6.44-7.89 (m, $\left.\mathrm{ArH}\right) ;{ }^{31} \mathrm{P}\left\{{ }^{1} \mathrm{H}\right\}$ NMR (161 MHz, $\left.\mathrm{CD}_{2} \mathrm{Cl}_{2}\right): \delta$ 50.18, 50.46; HRMS (ESI) $\mathrm{m} / z$ [positive mode] calcd. for $\mathrm{C}_{49} \mathrm{H}_{51} \mathrm{O}_{10} \mathrm{P}_{2} \mathrm{~S}_{2} 925.2399$, found 925.2399 . 
Secondary Phosphine: bis(2-methylphenyl)phosphine<smiles></smiles>

Figure S8. Structure of 3ad/3ad' (reported as an inseparable diastereomeric mixture)

White Solid; Eluent system (PTLC): DCM/Hex/MeOH (99.5:99.5:1) (provides pure chiral-3ad / meso-3ad' mixture); ${ }^{1} \mathrm{H} \mathrm{NMR}\left(400 \mathrm{MHz}, \mathrm{CD}_{2} \mathrm{Cl}_{2}\right): \delta 1.92\left(\mathrm{~s},-\mathrm{CH}_{3^{-}}\right), 1.94\left(\mathrm{~s},-\mathrm{CH}_{3^{-}}\right), 2.10$ (s, $\mathrm{CH}_{3^{-}}$), 3.19-3.24 (m, - $\left.\mathrm{CH}-\right), 3.65$ (s, - $\left.\mathrm{CH}_{3^{-}}\right), 3.68$ (s, - $\left.\mathrm{CH}_{3^{-}}\right), 3.71$ (s, - $\left.\mathrm{CH}_{3^{-}}\right), 4.52-4.68$ ((m, $\mathrm{CH}_{2}$ ), 5.37 (s, - $\mathrm{OH}-$ ), 6.42-8.02 (m, $\left.\mathrm{ArH}\right) ;{ }^{31} \mathrm{P}\left\{{ }^{1} \mathrm{H}\right\}$ NMR $\left(161 \mathrm{MHz}, \mathrm{CD}_{2} \mathrm{Cl}_{2}\right.$ ): $\delta 47.15$ (overlap of two signals); HRMS (ESI) $\mathrm{m} / z$ [positive mode] calcd. for $\mathrm{C}_{49} \mathrm{H}_{51} \mathrm{O}_{6} \mathrm{P}_{2} \mathrm{~S}_{2} 861.2602$, found 861.2602 .

Secondary Phosphine: bis(3,5-trifluoromethylphenyl)phosphine

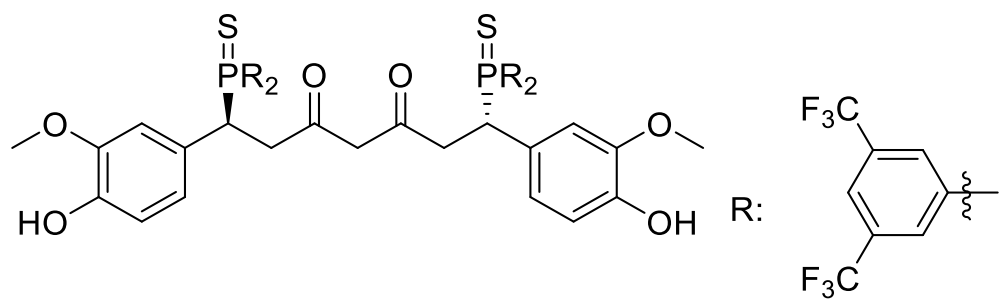

Figure S9. Structure of 3ae

White Solid; Eluent system (PTLC): DCM/Hex/MeOH (70:30:0.5); Yield: $26.1 \mathrm{mg}(23 \%) ;{ }^{1} \mathrm{H}$ NMR $\left(400 \mathrm{MHz}, \mathrm{CD}_{2} \mathrm{Cl}_{2}\right): \delta 2.77\left(\mathrm{ddd}, 2 \mathrm{H}, J_{\mathrm{HH}}=16.3 \mathrm{~Hz}, J_{\mathrm{HP}}=12.6 \mathrm{~Hz}, J_{\mathrm{HH}}=4.0 \mathrm{~Hz}\right.$, PCHCHHCO), 2.87-2.98 (m, 2H, $\mathrm{PCHCH}_{2} \mathrm{CO}$ ), 3.73 (s, 6H, $\mathrm{CH}_{3} \mathrm{OAr}$ ), 4.47 (ddd, 2H, $J_{\mathrm{HH}}=9.4$ $\left.\mathrm{Hz}, J_{\mathrm{HP}}=9.4 \mathrm{~Hz}, J_{\mathrm{HH}}=4.2 \mathrm{~Hz}, \mathrm{PCHCHHCO}\right), 5.61(\mathrm{~s}, 2 \mathrm{H}, \mathrm{OH}), 6.63-6.68(\mathrm{~m}, 5 \mathrm{H}, \mathrm{ArH}), 6.79$ (s, 2H, $\left.\mathrm{COCH}_{2} \mathrm{CO}\right), 7.85-7.91(\mathrm{~m}, 7 \mathrm{H}, \mathrm{ArH}), 8.08-8.16(\mathrm{~m}, 2 \mathrm{H} \mathrm{ArH}), 8.55\left(\mathrm{~d}, 4 \mathrm{H}, J_{\mathrm{HH}}=11.6 \mathrm{~Hz}\right.$, $\mathrm{ArH}) ;{ }^{13} \mathrm{C} \mathrm{NMR}\left(100 \mathrm{MHz}, \mathrm{CD}_{2} \mathrm{Cl}_{2}\right): \delta 39.44\left(\mathrm{~d}, J_{\mathrm{CP}}=3.9 \mathrm{~Hz}\right), 41.94(\mathrm{~s}), 42.47(\mathrm{~s}), 56.30(\mathrm{~s})$, $102.12(\mathrm{~s}), 112.24\left(\mathrm{~d}, J_{\mathrm{CP}}=4.5 \mathrm{~Hz}\right), 114.55\left(\mathrm{~d}, J_{\mathrm{CP}}=2.1 \mathrm{~Hz}\right), 121.77\left(\mathrm{~d}, J_{\mathrm{CP}}=14.1 \mathrm{~Hz}\right), 123.06$ $\left(\mathrm{d}, J_{\mathrm{CP}}=6.9 \mathrm{~Hz}\right), 124.56\left(\mathrm{~d}, J_{\mathrm{CF}}=28.4 \mathrm{~Hz}\right), 124.65\left(\mathrm{~d}, J_{\mathrm{CF}}=20.6 \mathrm{~Hz}\right), 126.00\left(\mathrm{~d}, J_{\mathrm{CP}}=3.2 \mathrm{~Hz}\right)$, $126.76\left(\mathrm{~d}, J_{\mathrm{CP}}=7.8 \mathrm{~Hz}\right), 131.80\left(\mathrm{~d}, J_{\mathrm{CP}}=12.0 \mathrm{~Hz}\right), 132.52\left(\mathrm{~d}, J_{\mathrm{CP}}=10.3 \mathrm{~Hz}\right), 133.16\left(\mathrm{~d}, J_{\mathrm{CF}}=\right.$ $189.9 \mathrm{~Hz}), 133.52(\mathrm{~s}), 133.97\left(\mathrm{~d}, J_{\mathrm{CF}}=189.5 \mathrm{~Hz}\right), 134.24(\mathrm{~s}), 146.46\left(\mathrm{~d}, J_{\mathrm{CP}}=2.7\right), 146.96(\mathrm{~d}$, $\left.J_{\mathrm{CP}}=1.9\right), 189.50\left(\mathrm{~d}, J_{\mathrm{CP}}=13.7\right) ;{ }^{31} \mathrm{P}\left\{{ }^{1} \mathrm{H}\right\} \mathrm{NMR}\left(161 \mathrm{MHz}, \mathrm{CD}_{2} \mathrm{Cl}_{2}\right): \delta 49.44 ;{ }^{19} \mathrm{~F} \mathrm{NMR}(376$ $\mathrm{MHz}, \mathrm{CD}_{2} \mathrm{Cl}_{2}$ ) $\delta-63.47$, -63.24; HRMS (ESI) $\mathrm{m} / \mathrm{z}$ [positive mode] calcd. for $\mathrm{C}_{53} \mathrm{H}_{35} \mathrm{~F}_{24} \mathrm{O}_{6} \mathrm{P}_{2} \mathrm{~S}_{2}$ 1349.0967, found 1349.1034. 
<smiles>[R2]P[C@@H](CC(=O)CC(=O)C[C@H](P=S)c1ccc(O)c(OC)c1)[PH]=S</smiles><smiles>[R2]PC(CC(=O)CC(=O)/C=C/c1ccc(O)c(OC)c1)c1ccc(O)c(OC)c1</smiles>

$\mathrm{R}$ :<smiles>Cc1cc(C(F)(F)F)cc(C(F)(F)F)c1</smiles>

Figure S10. Structure of 2ae/3ae' (reported as an inseparable mixture with mono product)

Yellowish white Solid; Eluent system (PTLC): DCM/Hex/MeOH (70:30:0.5) (provides pure mono-2ae and meso-3ae' / mixture); ${ }^{1} \mathrm{H}$ NMR (400 MHz, $\left.\mathrm{CD}_{2} \mathrm{Cl}_{2}\right):{ }^{1} \mathrm{H} \mathrm{NMR}\left(400 \mathrm{MHz}, \mathrm{CD}_{2} \mathrm{Cl}_{2}\right)$ : $\delta 2.74\left(\mathrm{ddd}, 2 \mathrm{H}, J_{\mathrm{HH}}=16.2 \mathrm{~Hz}, J_{\mathrm{HP}}=11.9 \mathrm{~Hz}, J_{\mathrm{HH}}=4.4 \mathrm{~Hz}, \mathrm{PCHCHHCO}\right), 2.88-2.97(\mathrm{~m}, 2 \mathrm{H}$, $\mathrm{PCHCH}_{2} \mathrm{CO}$ ), 3.69 (s, 6H, CH $\mathrm{CHAr}_{3} \mathrm{OA}$, 4.42-4.48 (m, 2H, PCHCHHCO), 5.64 (s, 2H, OH), 6.606.75 (m, 7H, $\left.\mathrm{ArH}, \mathrm{COCH}_{2} \mathrm{CO}\right), 7.86-7.91$ (m, 7H, $\left.\mathrm{ArH}\right), 8.10-8.12$ (m, 2H ArH), 8.57 (d, 4H, $\left.J_{\mathrm{HH}}=11.6 \mathrm{~Hz}, \mathrm{ArH}\right) ;{ }^{31} \mathrm{P}\left\{{ }^{1} \mathrm{H}\right\} \mathrm{NMR}\left(161 \mathrm{MHz}, \mathrm{CD}_{2} \mathrm{Cl}_{2}\right): \delta 49.14$ (meso); ${ }^{19} \mathrm{~F} \mathrm{NMR}(376 \mathrm{MHz}$, $\left.\mathrm{CD}_{2} \mathrm{Cl}_{2}\right) \delta-63.48,-63.33$; HRMS (ESI) $\mathrm{m} / \mathrm{z}$ [positive mode] calcd. for $\mathrm{C}_{53} \mathrm{H}_{35} \mathrm{~F}_{24} \mathrm{O}_{6} \mathrm{P}_{2} \mathrm{~S}_{2}$ 1349.0967, found 1349.1006, $\mathrm{C}_{37} \mathrm{H}_{28} \mathrm{~F}_{12} \mathrm{O}_{6} \mathrm{PS} 859.1153$, found 859.1172. 


\section{B3 One-Pot Direct Dihydrophosphination-Complexation of Diphosphines}
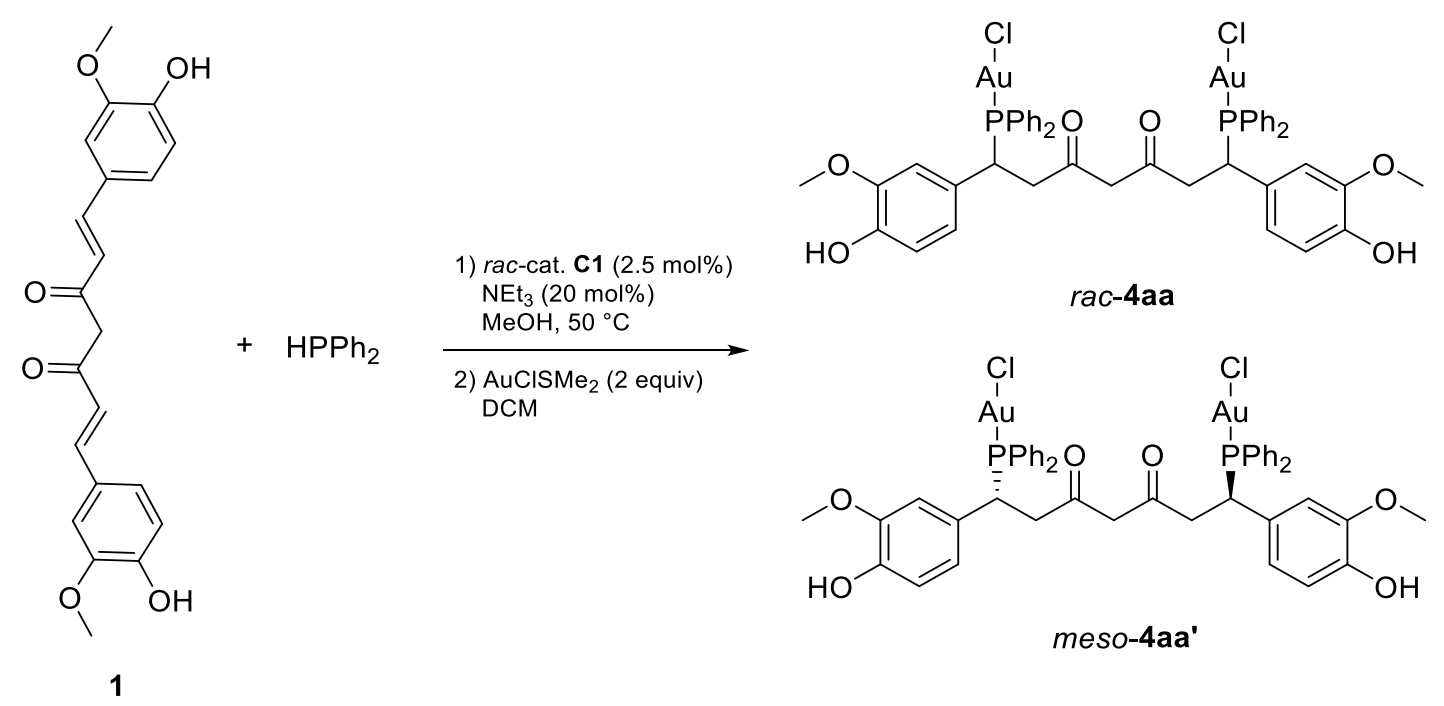

Scheme S2. Structure of Complexation of Diphosphines.

To a degassed solution $(7.5 \mathrm{~mL})$ of diphenylphosphine $(52.1 \mathrm{mg}, 0.28 \mathrm{mmol})$ was added raccatalyst C1. Curcumin (51.6 mg, $0.14 \mathrm{mmol})$ and $\mathrm{NEt}_{3}(5.7 \mathrm{mg}, 0.056 \mathrm{mmol})$ were subsequently added into the reaction mixture. The reaction mixture was then stirred at the $50{ }^{\circ} \mathrm{C}$ for $48 \mathrm{~h}$. $\mathrm{AuClSMe}_{2}(82.5 \mathrm{mg}, 0.28 \mathrm{mmol})$ was added to the crude mixture and stirred at ambient temperature for $8 \mathrm{~h}$. The solution was purified directly by column chromatography on silica gel with 99:1 dichloromethane/ethyl acetate as the eluent to provide the products.

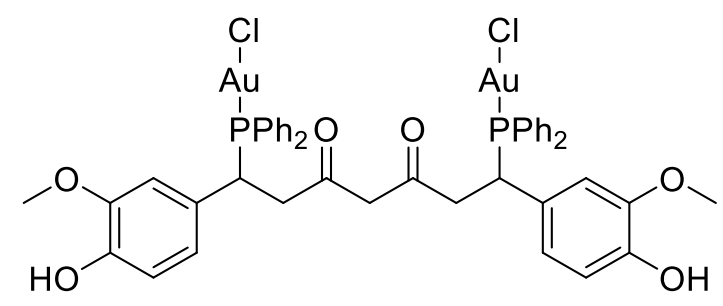

Figure S11. rac-4aa

White Solid; Eluent system (column chromatography): DCM/EA (99:1); Yield: $141.8 \mathrm{mg}$ (42\%); ${ }^{1} \mathrm{H}$ NMR $\left(400 \mathrm{MHz}, \mathrm{CD}_{2} \mathrm{Cl}_{2}\right): \delta 2.72\left(\mathrm{ddd}, 2 \mathrm{H}, J_{\mathrm{HH}}=15.6 \mathrm{~Hz}, J_{\mathrm{HP}}=10.2 \mathrm{~Hz}, J_{\mathrm{HH}}=3.5 \mathrm{~Hz}\right.$, $\mathrm{PCHCHHCO}), 2.97\left(\mathrm{ddd}, 2 \mathrm{H}, J_{\mathrm{HH}}=15.7 \mathrm{~Hz}, J_{\mathrm{HP}}=11.0 \mathrm{~Hz}, J_{\mathrm{HH}}=8.6 \mathrm{~Hz}, \mathrm{PCHCH}_{2} \mathrm{CO}\right), 3.77(\mathrm{~s}$, $6 \mathrm{H}, \mathrm{CH}_{3} \mathrm{OAr}$ ), $4.28\left(\mathrm{ddd}, 2 \mathrm{H}, J_{\mathrm{HH}}=12.7 \mathrm{~Hz}, J_{\mathrm{HP}}=12.7 \mathrm{~Hz}, J_{\mathrm{HH}}=3.4 \mathrm{~Hz}, \mathrm{PCHCHHCO}\right), 5.63(\mathrm{~s}$, $2 \mathrm{H}, \mathrm{OH}), 6.50-6.54(\mathrm{~m}, 2 \mathrm{H}, \mathrm{ArH}), 6.61$ (s, 1H, $\left.\mathrm{COCH}_{2} \mathrm{CO}\right), 6.63$ (s, 1H, $\left.\mathrm{COCH}_{2} \mathrm{CO}\right), 6.80-6.87$ $(\mathrm{m}, 2 \mathrm{H}, \mathrm{ArH}), 7.27-7.41(\mathrm{~m}, 11 \mathrm{H} \mathrm{ArH}), 7.50-7.62(\mathrm{~m}, 7 \mathrm{H}, \mathrm{ArH}), 7.87-7.94(\mathrm{~m}, 4 \mathrm{H}, \mathrm{ArH}) ;{ }^{13} \mathrm{C}$ NMR (100 MHz, $\mathrm{CD}_{2} \mathrm{Cl}_{2}$ ): $\delta 40.40\left(\mathrm{t}, J_{\mathrm{CP}}=5.4 \mathrm{~Hz}\right.$ ), $40.76(\mathrm{~s}), 56.14(\mathrm{~s}), 101.64$ (s), 111.94 (d, 
$\left.J_{\mathrm{CP}}=6.4 \mathrm{~Hz}\right), 114.20\left(\mathrm{~d}, J_{\mathrm{CP}}=2.4 \mathrm{~Hz}\right), 122.89\left(\mathrm{~d}, J_{\mathrm{CP}}=6.4 \mathrm{~Hz}\right), 125.99(\mathrm{~s}), 127.08(\mathrm{~s}), 127.62$ $(\mathrm{s}), 128.76\left(\mathrm{~d}, J_{\mathrm{CP}}=11.6 \mathrm{~Hz}\right), 129.58\left(\mathrm{~d}, J_{\mathrm{CP}}=11.1 \mathrm{~Hz}\right), 131.93\left(\mathrm{~d}, J_{\mathrm{CP}}=2.0 \mathrm{~Hz}\right), 132.62\left(\mathrm{~d}, J_{\mathrm{CP}}\right.$ $=2.7 \mathrm{~Hz}), 133.90\left(\mathrm{~d}, J_{\mathrm{CP}}=12.8 \mathrm{~Hz}\right), 134.30\left(\mathrm{~d}, J_{\mathrm{CP}}=13.2 \mathrm{~Hz}\right), 145.39\left(\mathrm{~d}, J_{\mathrm{CP}}=3.3 \mathrm{~Hz}\right), 146.81$ $\left(\mathrm{d}, J_{\mathrm{CP}}=2.1 \mathrm{~Hz}\right), 189.35\left(\mathrm{~d}, J_{\mathrm{CP}}=16.1\right) ;{ }^{31} \mathrm{P}\left\{{ }^{1} \mathrm{H}\right\} \mathrm{NMR}\left(161 \mathrm{MHz}, \mathrm{CD}_{2} \mathrm{Cl}_{2}\right): \delta 45.11 ;$ HRMS (ESI) $\mathrm{m} / \mathrm{z}$ [positive mode] calcd. for $\mathrm{C}_{45} \mathrm{H}_{43} \mathrm{O}_{6} \mathrm{P}_{2} \mathrm{Cl}_{2} \mathrm{Au}_{2}$ 1205.1243, found 1205.1243.

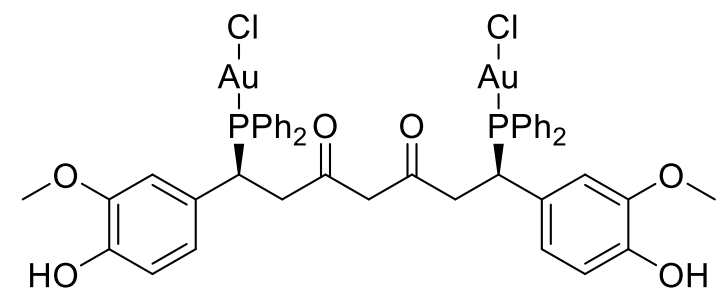

Figure S12. meso-4aa'

White Solid; Eluent system (column chromatography): DCM/EA (99:1); Yield: $108.0 \mathrm{mg}$ (32\%); ${ }^{1} \mathrm{H}$ NMR $\left(400 \mathrm{MHz}, \mathrm{CD}_{2} \mathrm{Cl}_{2}\right): \delta$ 2.69-2.79 (m, 2H, PCHCHHCO), 2.91-3.00 (m, 2H, PCHCH$H_{2} \mathrm{CO}$ ), 3.74 (s, $\left.6 \mathrm{H}, \mathrm{CH}_{3} \mathrm{OAr}\right), 4.23-4.30(\mathrm{~m}, 2 \mathrm{H}, \mathrm{PCHCHHCO}), 5.68$ (bs, 2H, OH), 6.47$6.68(\mathrm{~m}, 4 \mathrm{H}, \mathrm{ArH}), 6.82\left(\mathrm{~s}, 2 \mathrm{H}, \mathrm{COCH}_{2} \mathrm{CO}\right), 7.28-7.41(\mathrm{~m}, 12 \mathrm{H}, \mathrm{Ar} H), 7.55-7.56(\mathrm{~m}, 6 \mathrm{H} \mathrm{ArH})$, 7.91-7.95 (m, 4H, $\mathrm{Ar} H) ;{ }^{13} \mathrm{C}$ NMR (100 MHz, $\left.\mathrm{CD}_{2} \mathrm{Cl}_{2}\right): \delta 40.77(\mathrm{~s}), 41.14(\mathrm{~s}), 56.42(\mathrm{~s}), 102.05$ $(\mathrm{s}), 112.14\left(\mathrm{~d}, J_{\mathrm{CP}}=6.3 \mathrm{~Hz}\right), 114.62(\mathrm{~s}), 123.16\left(\mathrm{~d}, J_{\mathrm{CP}}=6.5 \mathrm{~Hz}\right), 126.26(\mathrm{~s}), 127.53(\mathrm{~s}), 128.02$ $\left(\mathrm{d}, J_{\mathrm{CP}}=9.6 \mathrm{~Hz}\right), 129.10\left(\mathrm{~d}, J_{\mathrm{CP}}=11.7 \mathrm{~Hz}\right), 129.94\left(\mathrm{~d}, J_{\mathrm{CP}}=11.1 \mathrm{~Hz}\right), 132.28\left(\mathrm{~d}, J_{\mathrm{CP}}=2.3 \mathrm{~Hz}\right)$, $132.95\left(\mathrm{~d}, J_{\mathrm{CP}}=2.3 \mathrm{~Hz}\right), 134.25\left(\mathrm{~d}, J_{\mathrm{CP}}=12.8 \mathrm{~Hz}\right), 134.68\left(\mathrm{~d}, J_{\mathrm{CP}}=13.1 \mathrm{~Hz}\right), 145.69\left(\mathrm{~d}, J_{\mathrm{CP}}=\right.$ $3.3 \mathrm{~Hz}), 147.17\left(\mathrm{~d}, J_{\mathrm{CP}}=2.3 \mathrm{~Hz}\right), 189.71\left(\mathrm{~d}, J_{\mathrm{CP}}=16.4 \mathrm{~Hz}\right) ;{ }^{31} \mathrm{P}\left\{{ }^{1} \mathrm{H}\right\} \mathrm{NMR}\left(161 \mathrm{MHz}, \mathrm{CD}_{2} \mathrm{Cl}_{2}\right)$ : $\delta$ 44.99; HRMS (ESI) $\mathrm{m} / \mathrm{z}$ [positive mode] calcd. for $\mathrm{C}_{45} \mathrm{H}_{43} \mathrm{O}_{6} \mathrm{P}_{2} \mathrm{Cl}_{2} \mathrm{Au}_{2}$ 1205.1243, found 1205.1243 . 


\section{NMR SPECTROSCOPY DATA}

C1 Mono-Hydrophosphinated Curcumin Sulfide mono-2aa

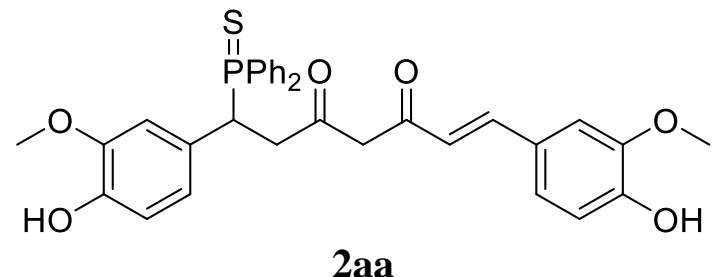

${ }^{1}$ H NMR spectrum

(400 MHz, $\mathrm{CD}_{2} \mathrm{Cl}_{2}$ )

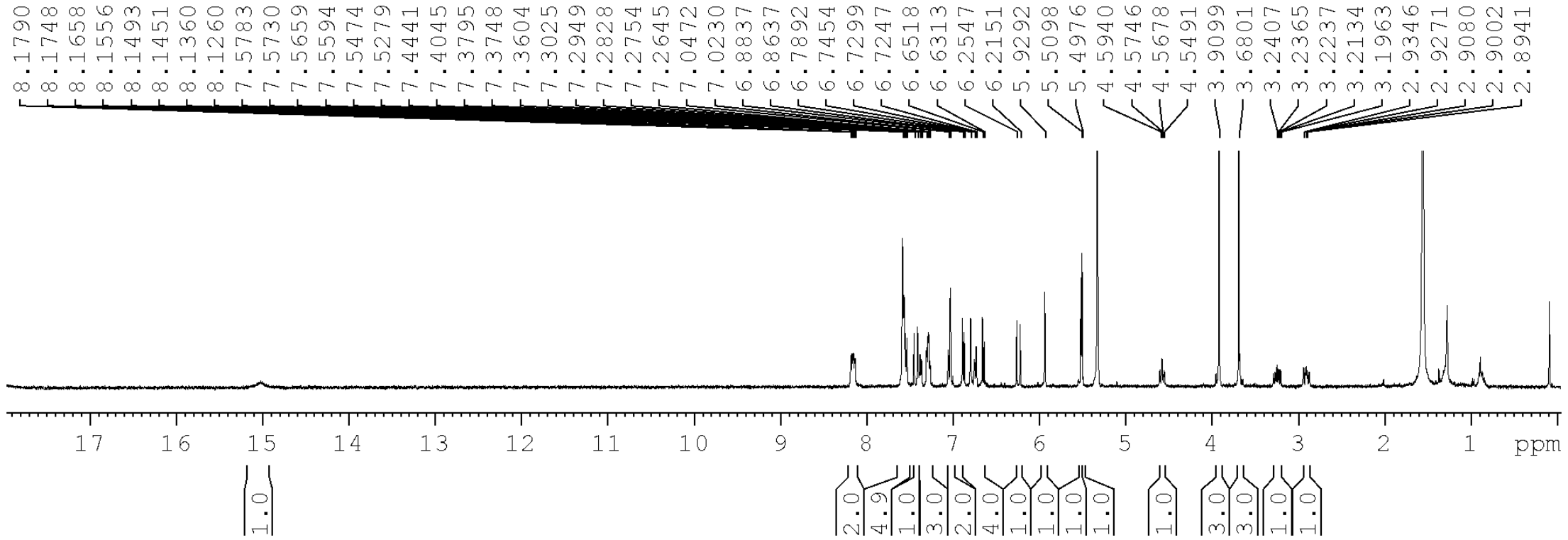

Figure S13. ${ }^{1} \mathrm{H}$ NMR spectrum of $\mathbf{2 a a}$ 


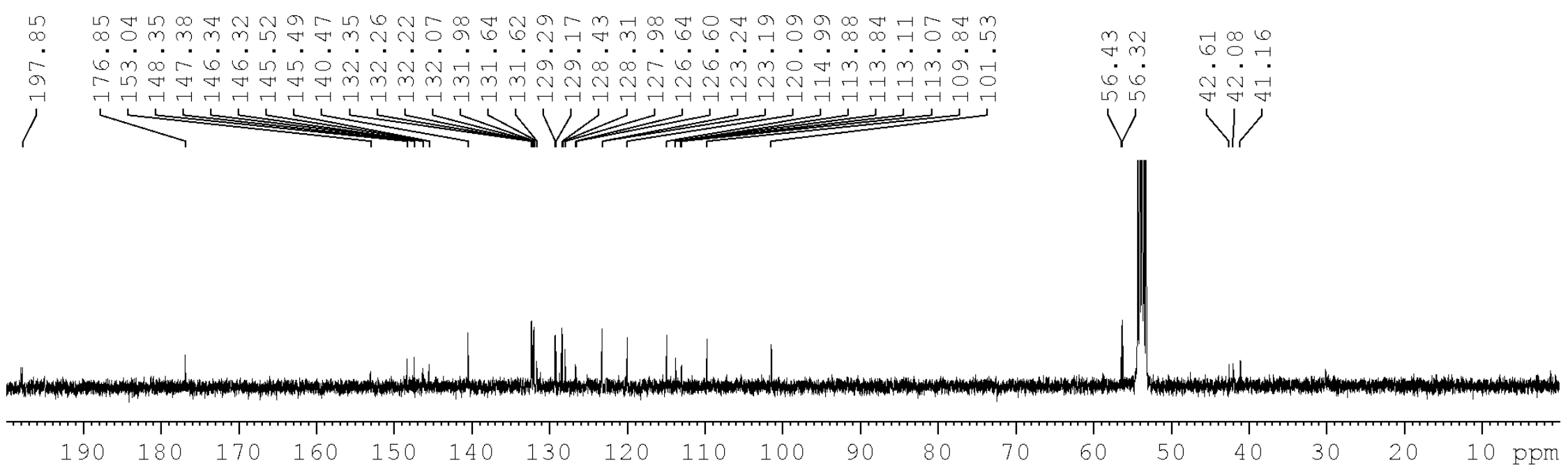

Figure S14. ${ }^{13} \mathrm{C}$ NMR spectrum of 2 aa 


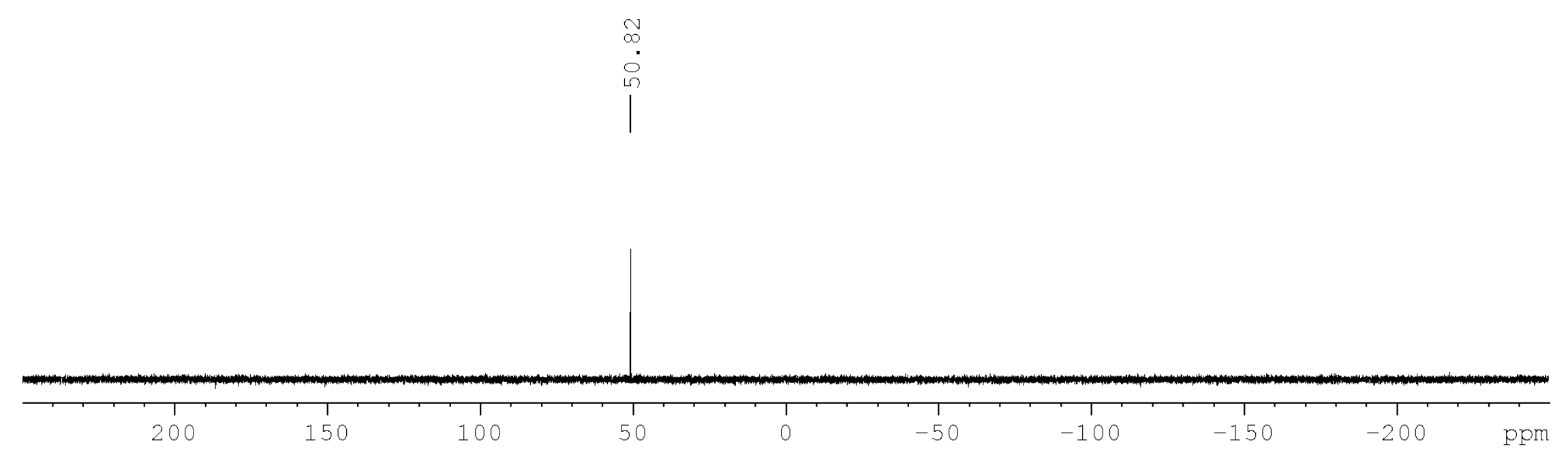

Figure S15. ${ }^{31} \mathrm{P}\left\{{ }^{1} \mathrm{H}\right\}$ NMR spectrum of 2aa 


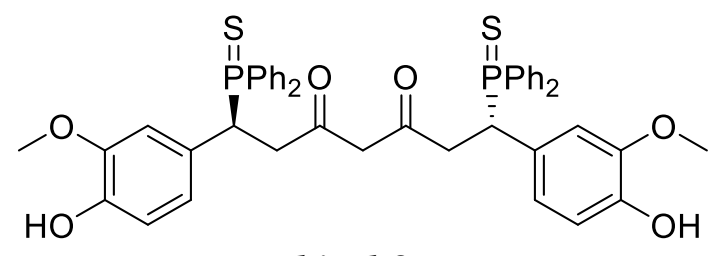

\section{chiral-3aa}

(Note: Stereochemistry of compound determined by single crystal $x$-ray crystallography)

\section{${ }^{1}$ H NMR spectrum}

$\left(400 \mathrm{MHz}, \mathrm{CD}_{2} \mathrm{Cl}_{2}\right)$

m小

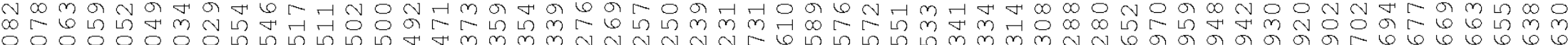

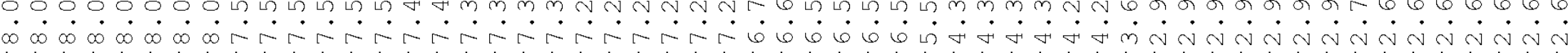
TIN
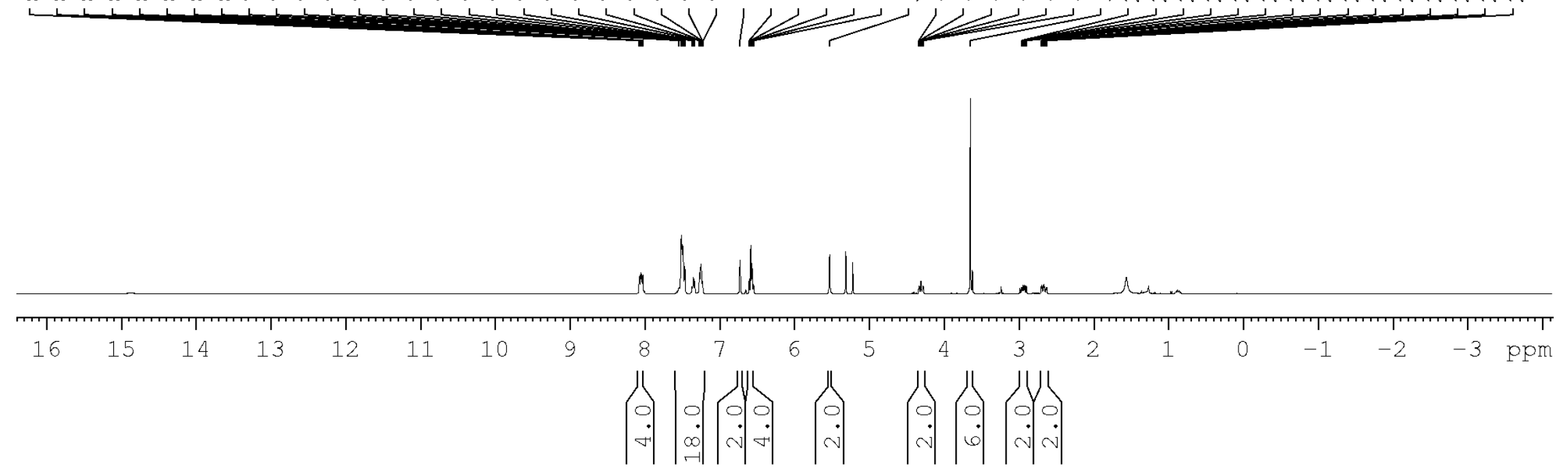

Figure S16. ${ }^{1} \mathrm{H}$ NMR spectrum of chiral-3aa 


\section{${ }^{13}$ C NMR spectrum}

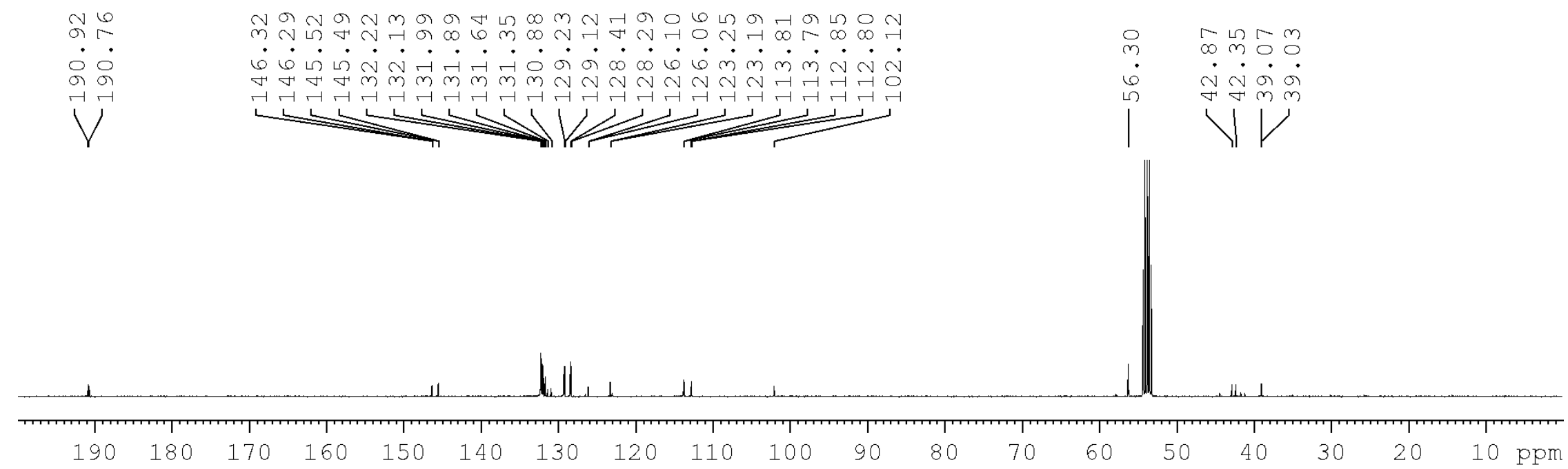

Figure S17. ${ }^{13} \mathrm{C}$ NMR spectrum of chiral-3aa 


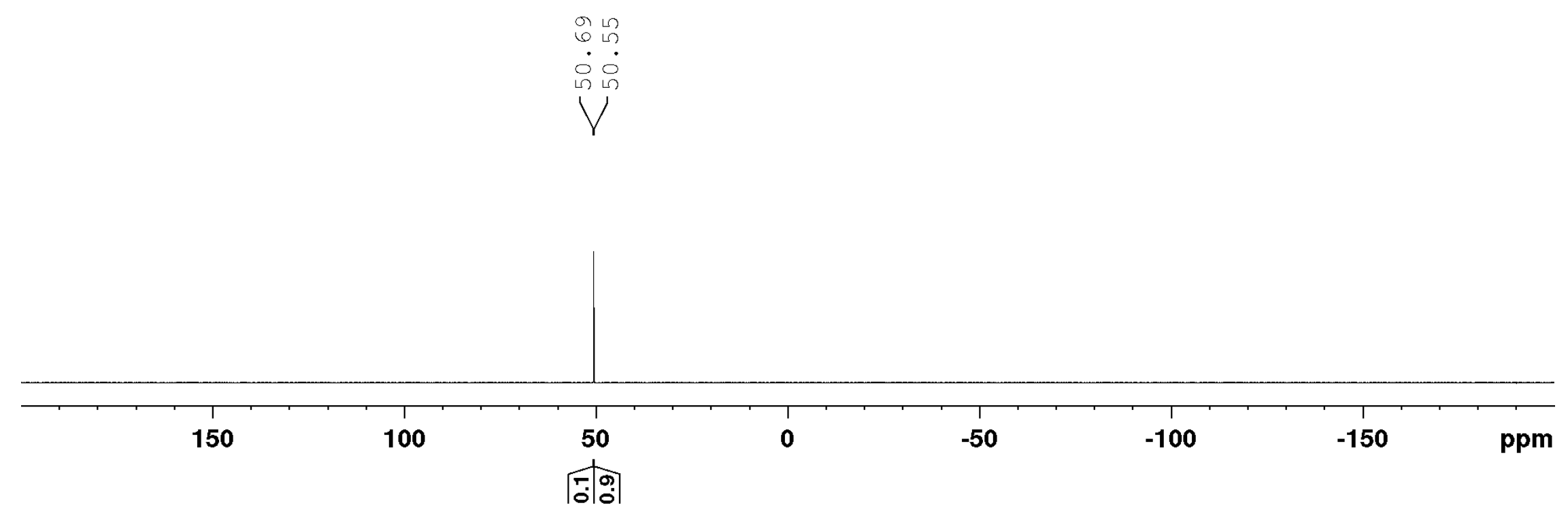

Figure S18. ${ }^{31} \mathrm{P}\left\{{ }^{1} \mathrm{H}\right\}$ NMR spectrum of chiral-3aa 


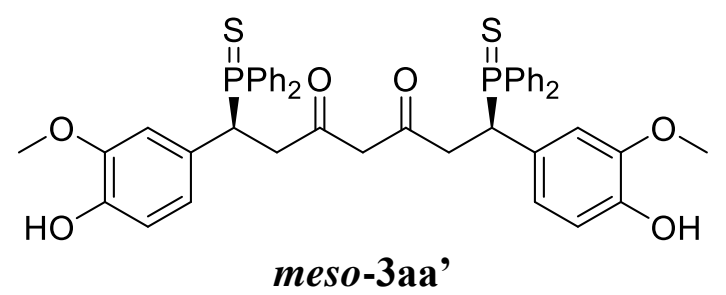

\section{${ }^{1}$ H NMR spectrum}

$\left(400 \mathrm{MHz}, \mathrm{CD}_{2} \mathrm{Cl}_{2}\right)$

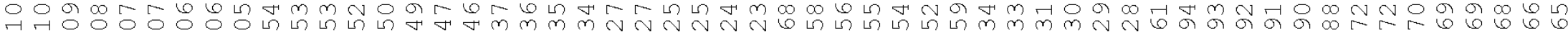

每

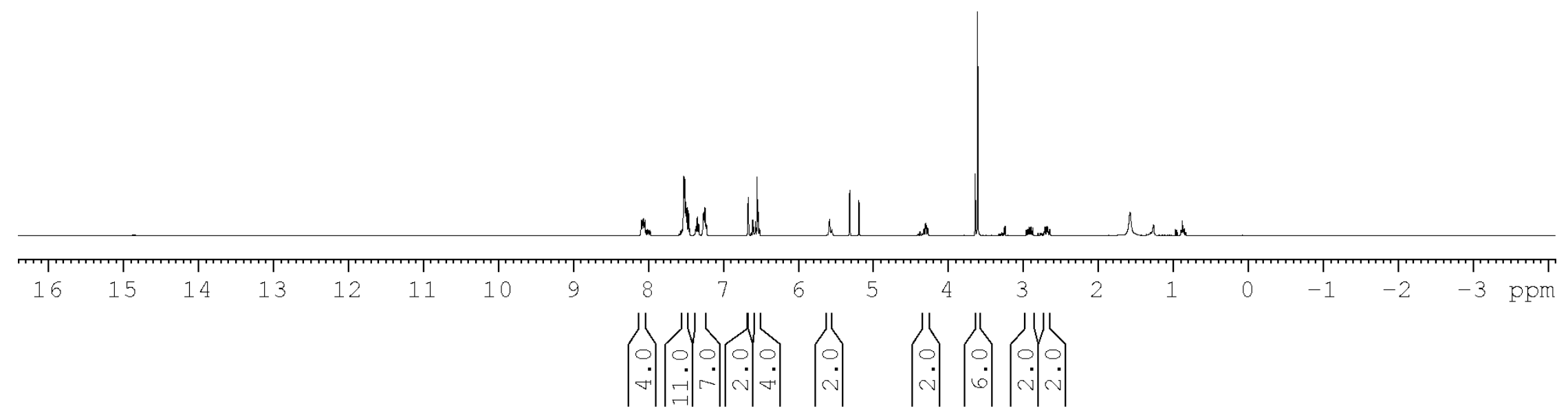

Figure S19. 'H NMR spectrum of meso-3aa' 
${ }^{13}$ C NMR spectrum

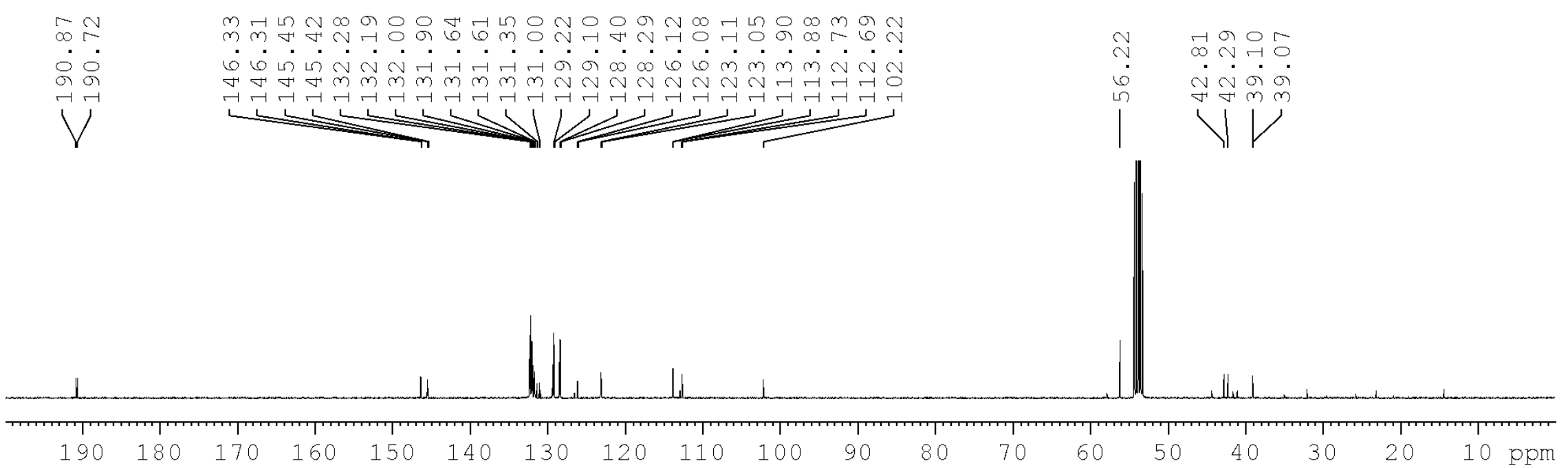

Figure S20. ${ }^{13} \mathrm{C}$ NMR spectrum of meso-3aa' 


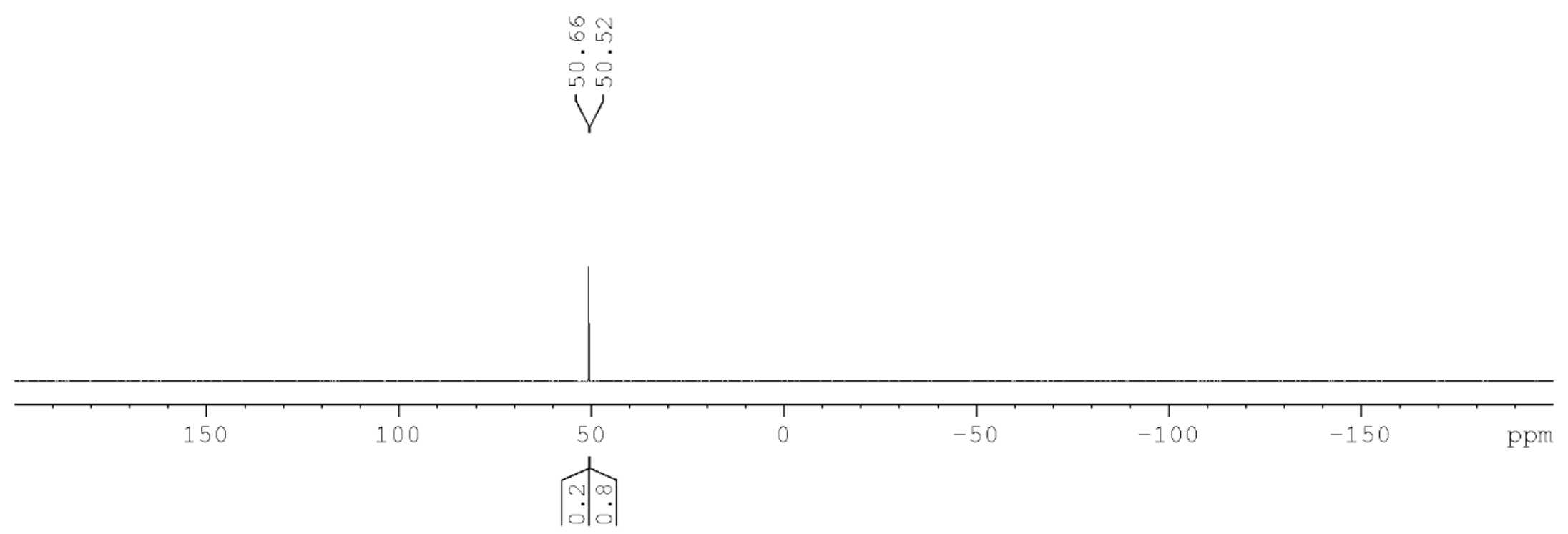

Figure S21. ${ }^{31} \mathrm{P}\left\{{ }^{1} \mathrm{H}\right\}$ NMR spectrum of meso-3aa' 


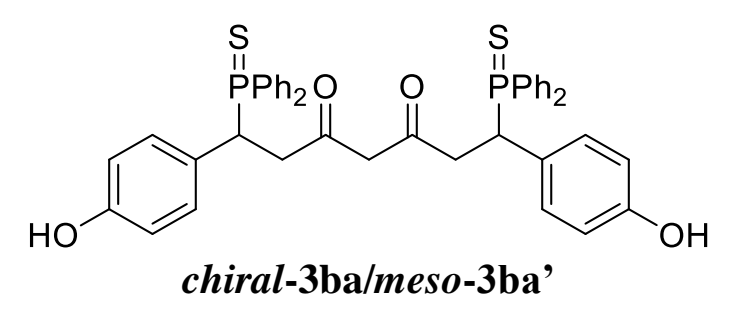

\section{${ }^{1}$ H NMR spectrum}

$\left(400 \mathrm{MHz}, \mathrm{CD}_{2} \mathrm{Cl}_{2}\right)$

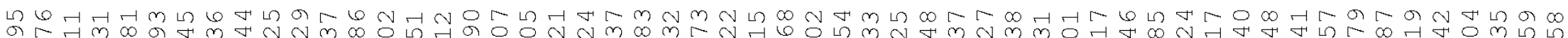

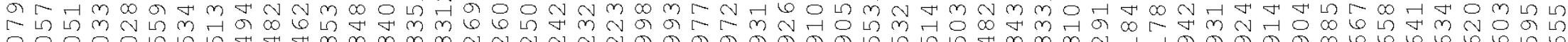
mmmmNNNNNGののの-

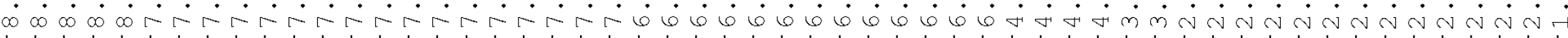

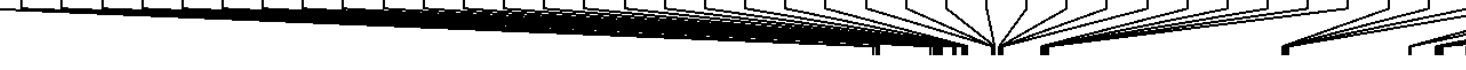

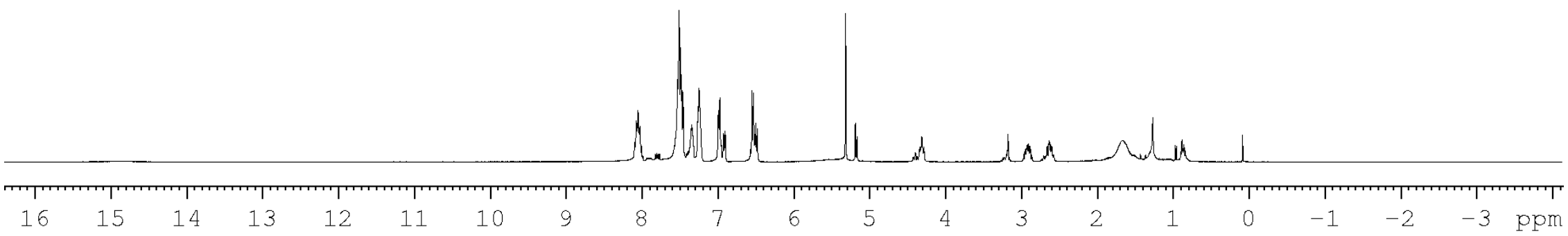

Figure S22. 'H NMR spectrum of chiral-3ba/meso-3ba' 
の $6 \infty \infty$

भ. भ.

능ㅇํ요용

4

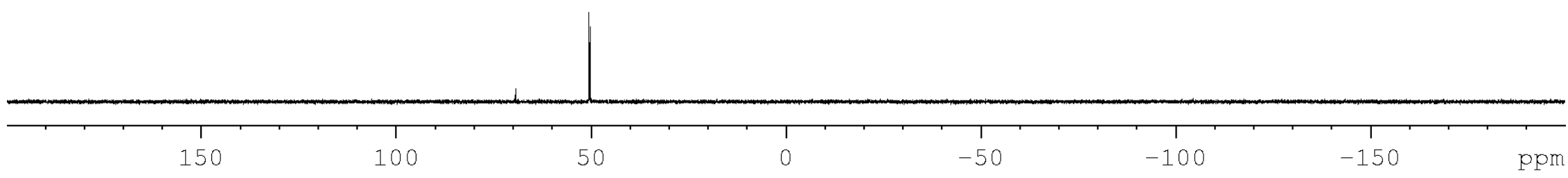

Figure S23. ${ }^{31} \mathrm{P}\left\{{ }^{1} \mathrm{H}\right\}$ NMR spectrum of chiral-3ba/meso-3ba' 


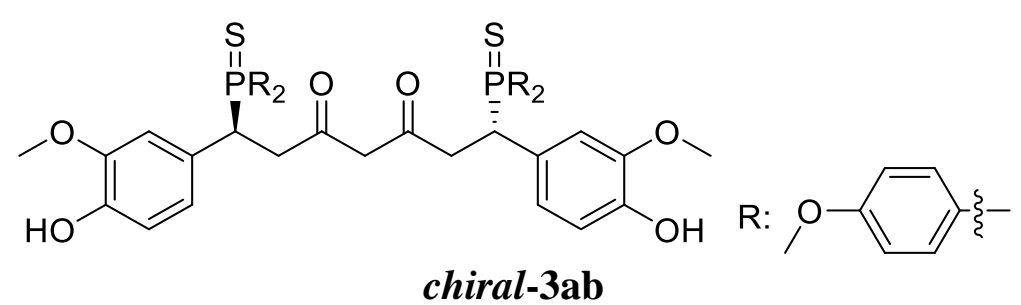

\section{${ }^{1}$ H NMR spectrum}

$\left(400 \mathrm{MHz}, \mathrm{CD}_{2} \mathrm{Cl}_{2}\right.$ )

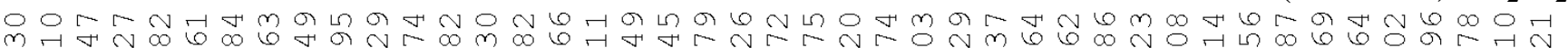
न

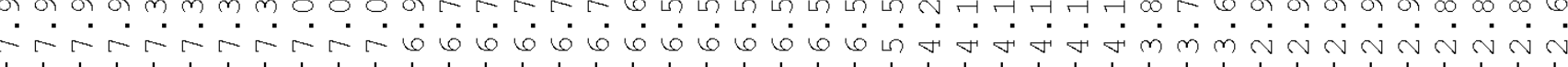
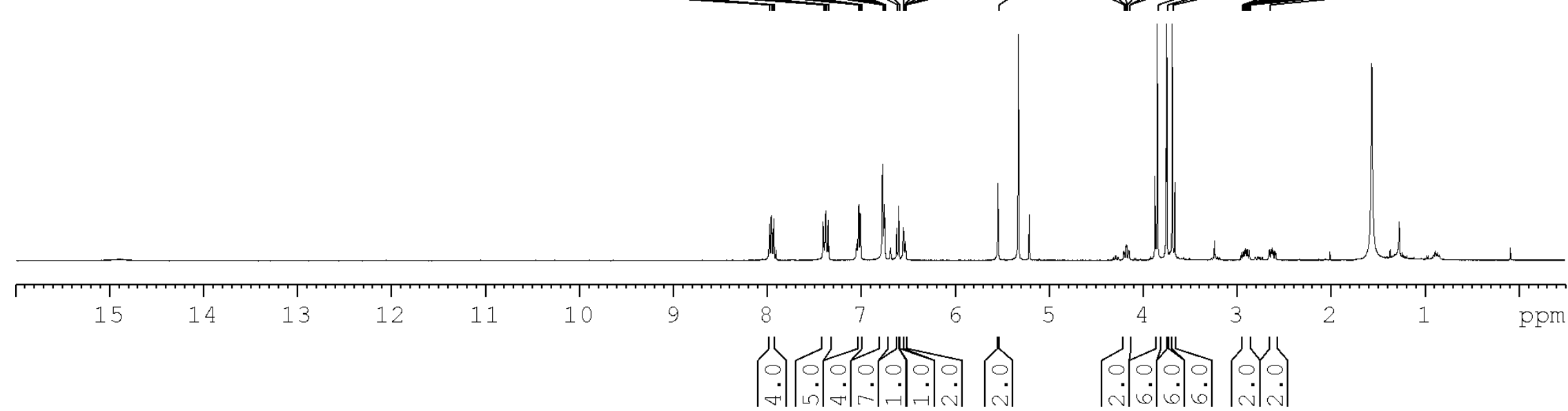

5

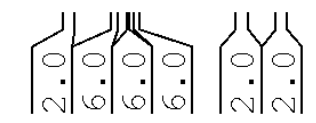

Figure S24. ${ }^{1} \mathrm{H}$ NMR spectrum of chiral-3ab 
${ }^{13}$ C NMR spectrum

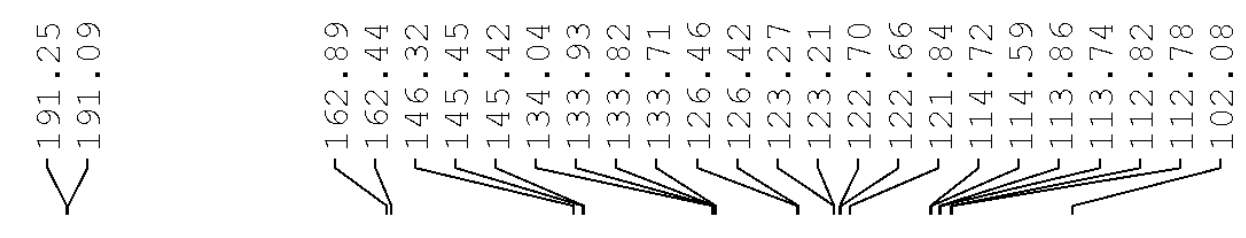

の

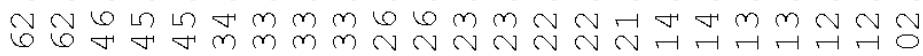

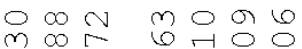

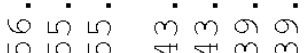

$\longrightarrow$

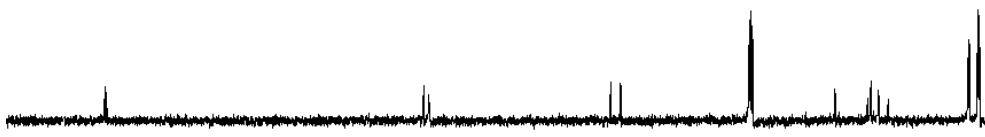

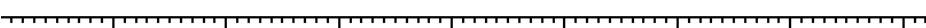

190

170

160

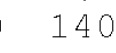

90

80

Figure S25. ${ }^{13} \mathrm{C}$ NMR spectrum of chiral-3ab 


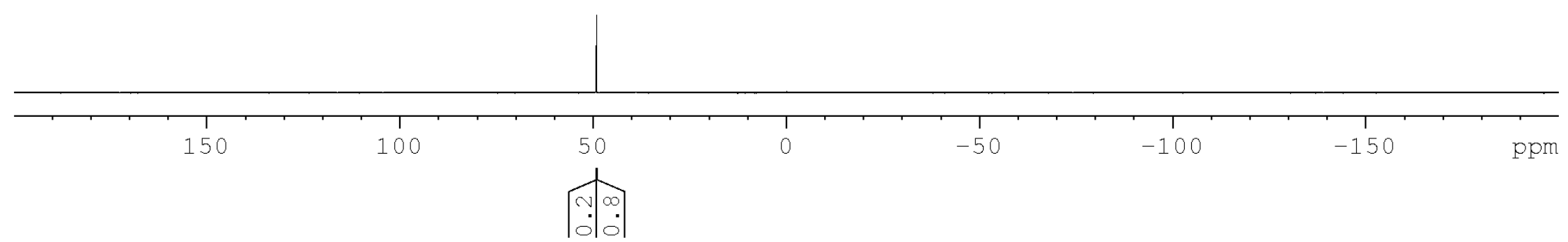

Figure S26. ${ }^{31} \mathrm{P}\left\{{ }^{1} \mathrm{H}\right\}$ NMR spectrum of chiral-3ab 


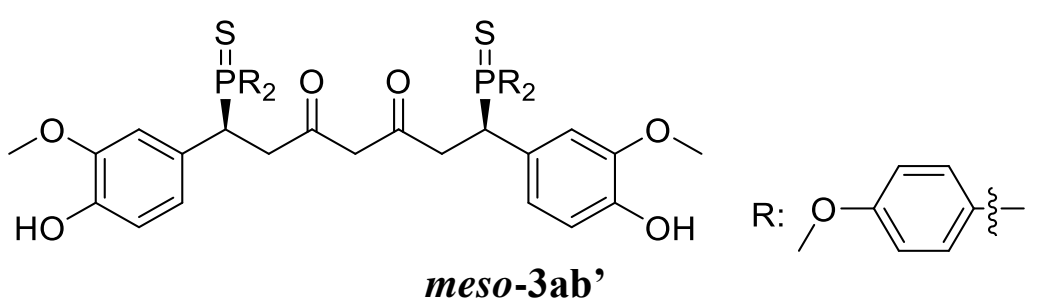

\section{${ }^{1}$ H NMR spectrum}

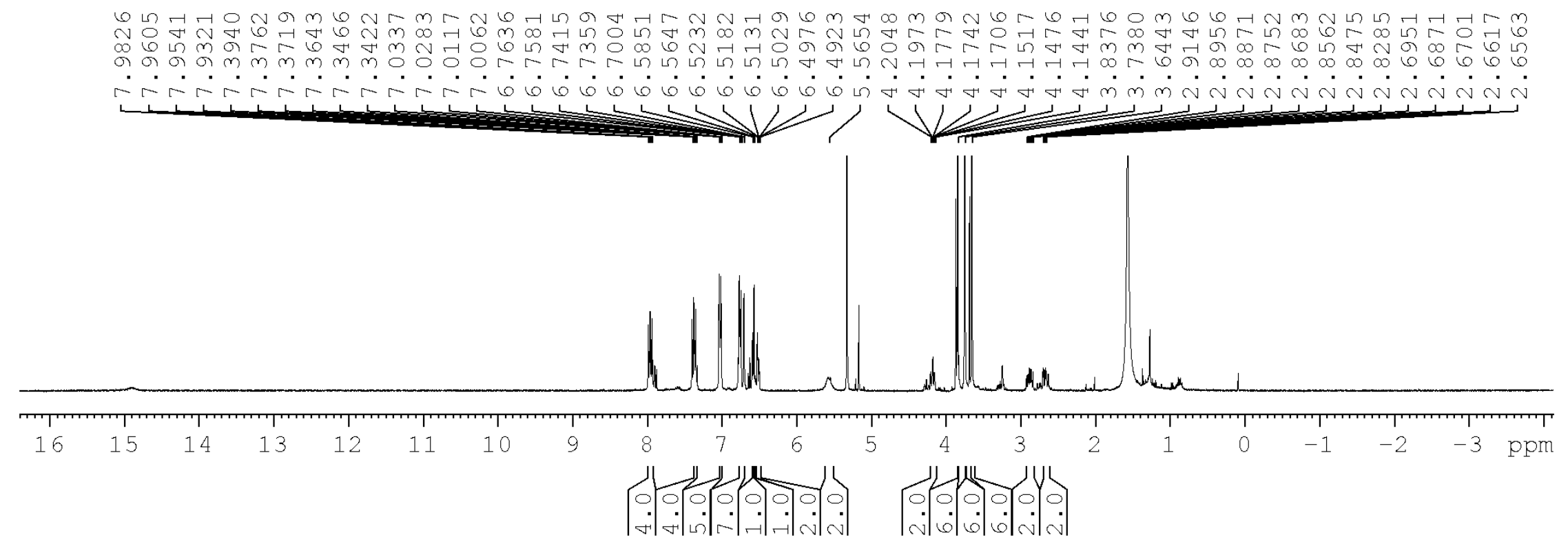

Figure S27. ${ }^{1} \mathrm{H}$ NMR spectrum of meso-3ab' 


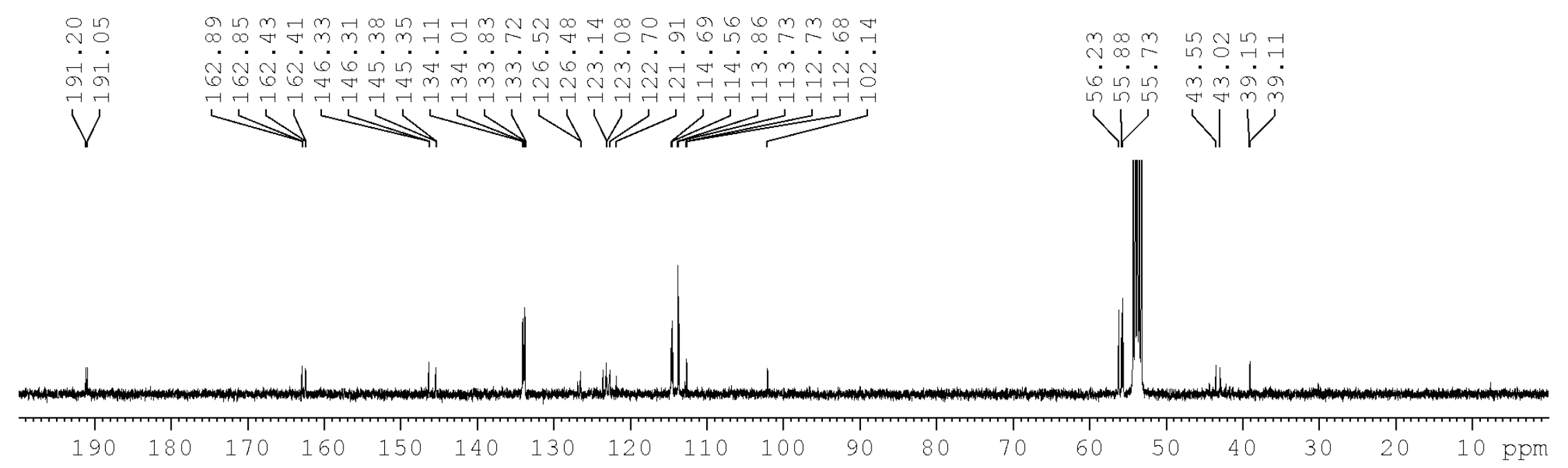

Figure S28. ${ }^{13} \mathrm{C}$ NMR spectrum of meso-3ab' 


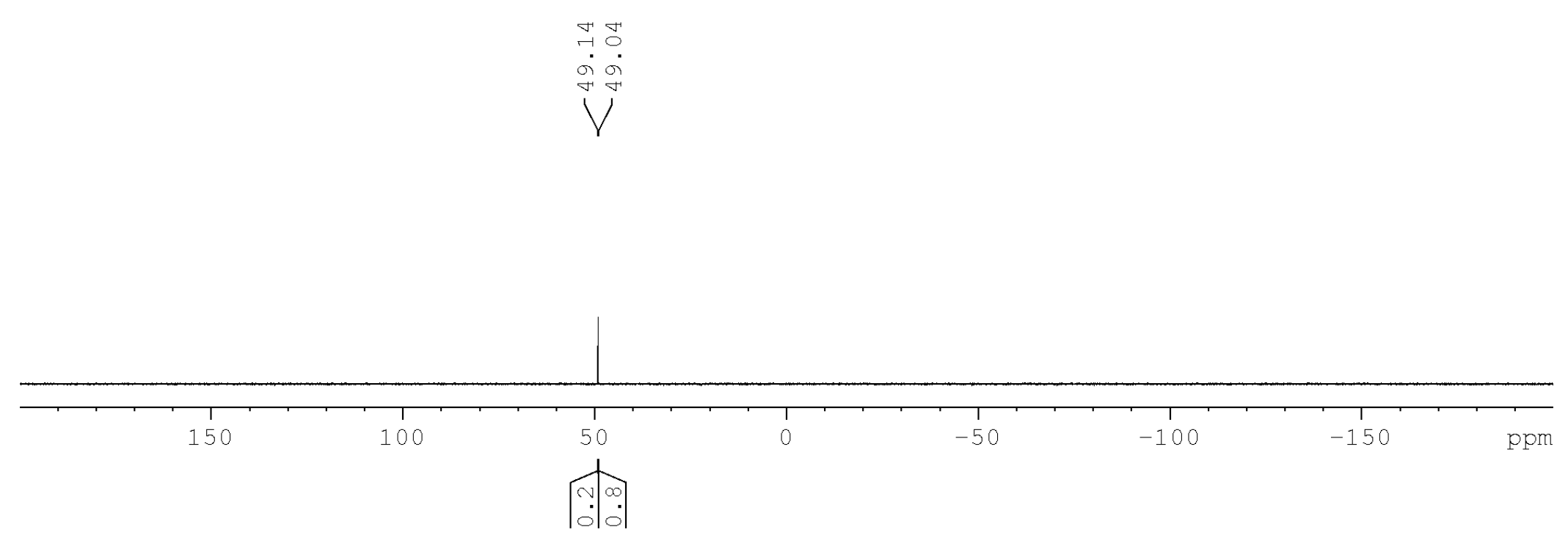

Figure S29. ${ }^{31} \mathrm{P}\left\{{ }^{1} \mathrm{H}\right\}$ NMR spectrum of meso-3ab' 


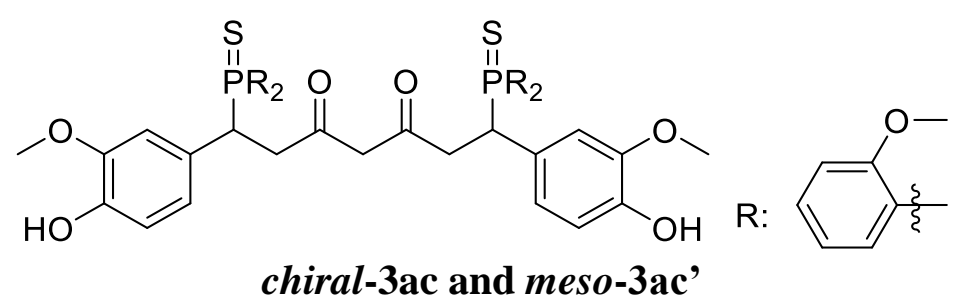

\section{${ }^{1}$ H NMR spectrum}
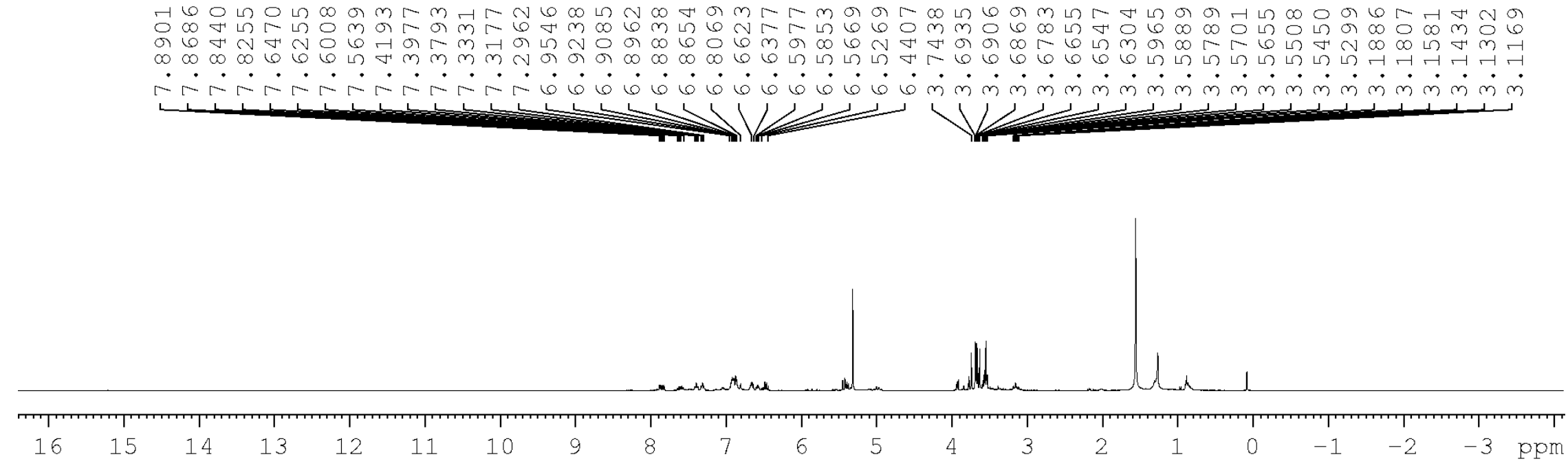

Figure S30. ${ }^{1} \mathrm{H}$ NMR spectrum of chiral-3ac/meso-3ac' 
$\cos 2$

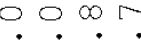

$\dot{m} \dot{m} \dot{\sim} \sim \vec{N}$

4

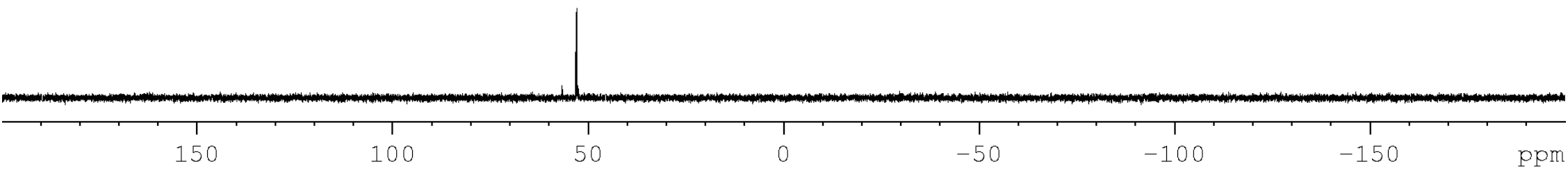

Figure S31. ${ }^{31} \mathrm{P}\left\{{ }^{1} \mathrm{H}\right\}$ NMR spectrum of chiral-3ac/meso-3ac' 


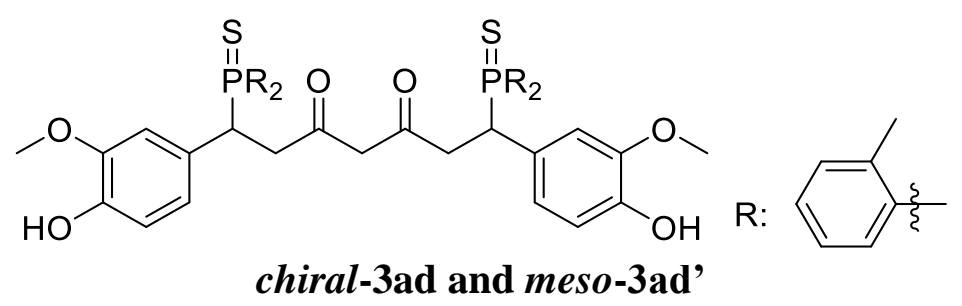

\section{${ }^{1}$ H NMR spectrum}

$\left(400 \mathrm{MHz}, \mathrm{CD}_{2} \mathrm{Cl}_{2}\right)$

m⿻上丨丶 갸의

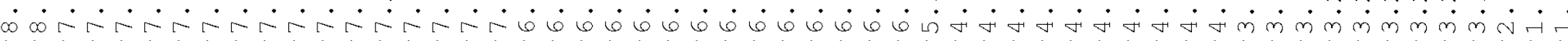

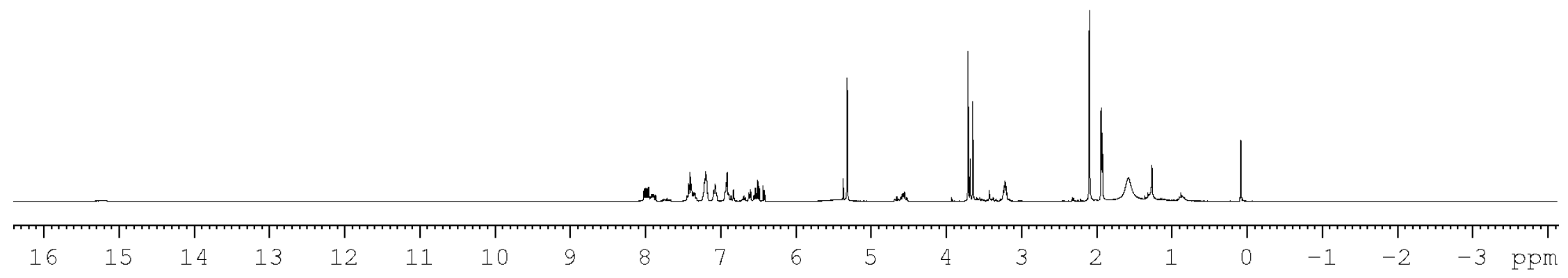

Figure S32. ${ }^{1} \mathrm{H}$ NMR spectrum of chiral-3ad/meso-3ad' 
$\therefore$

称

Y

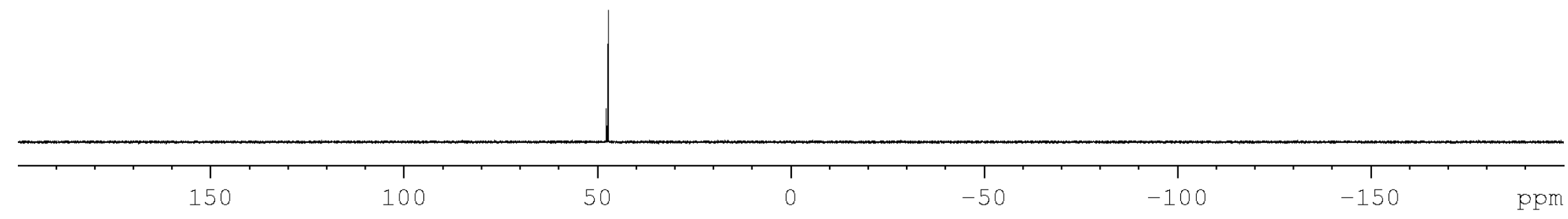

Figure S33. ${ }^{31} \mathrm{P}\left\{{ }^{1} \mathrm{H}\right\}$ NMR spectrum of chiral-3ad/meso-3ad' 


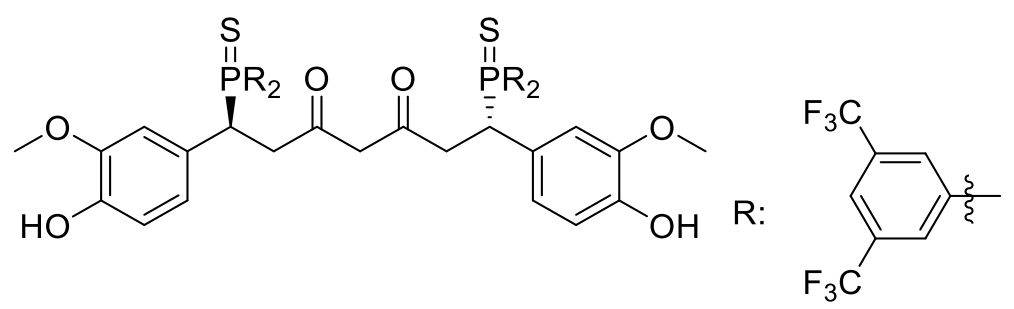

chiral-3ae

\section{${ }^{1}$ H NMR spectrum}

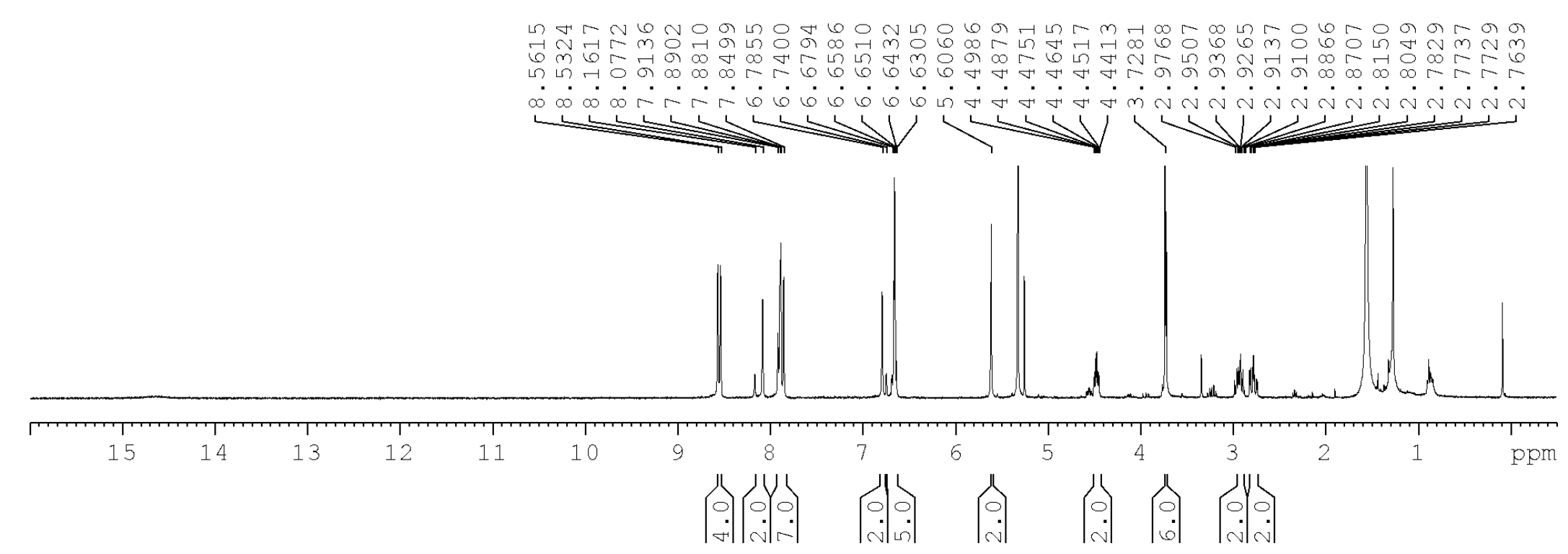

Figure S34. ${ }^{1} \mathrm{H}$ NMR spectrum of chiral-3ae 


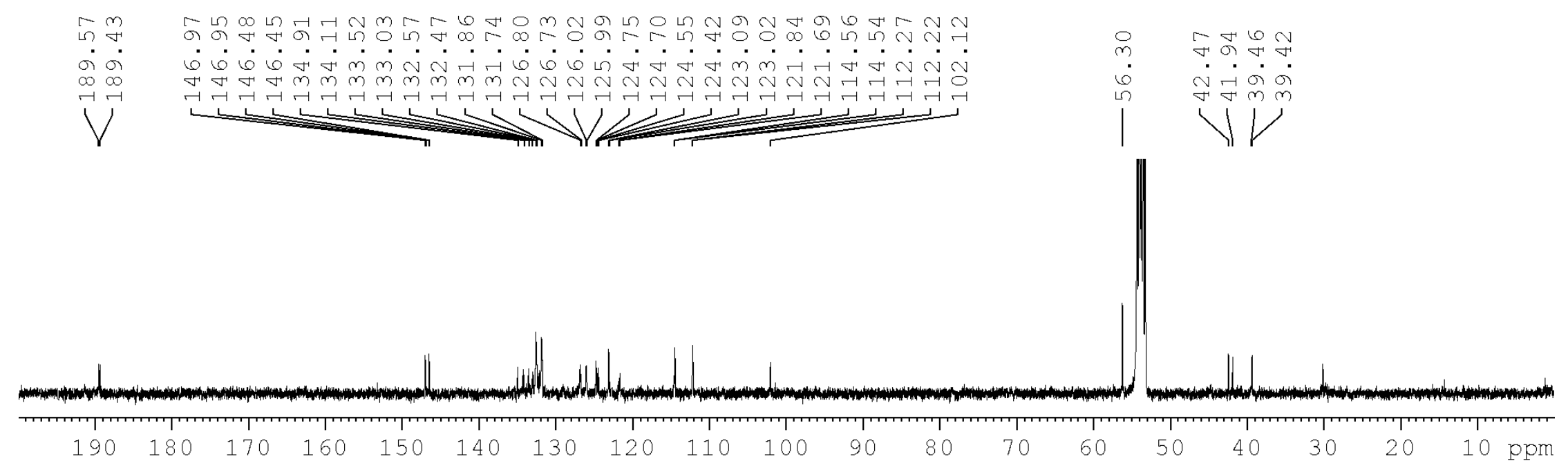

Figure S35. ${ }^{13} \mathrm{C}$ NMR spectrum of chiral-3ae 


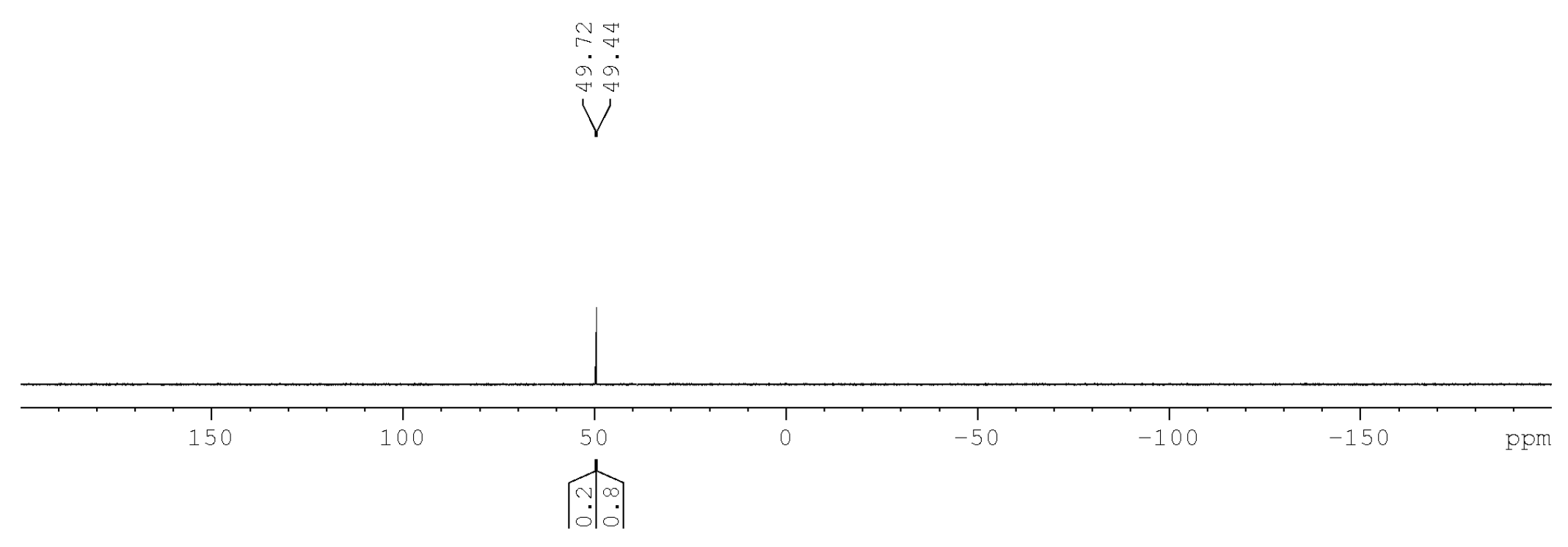

Figure S36. ${ }^{31} \mathrm{P}\left\{{ }^{1} \mathrm{H}\right\}$ NMR spectrum of chiral-3ae 
C10 Di-Hydrophosphinated Curcumin Sulfide mono-2ae and meso-3ae'

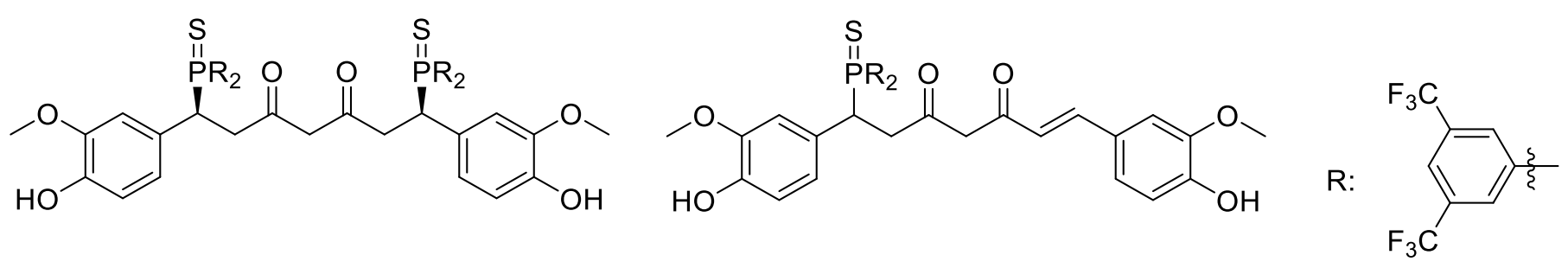

mono-2ae and meso-3ae'

${ }^{1}$ H NMR spectrum

(400 MHz, $\mathrm{CD}_{2} \mathrm{Cl}_{2}$ )
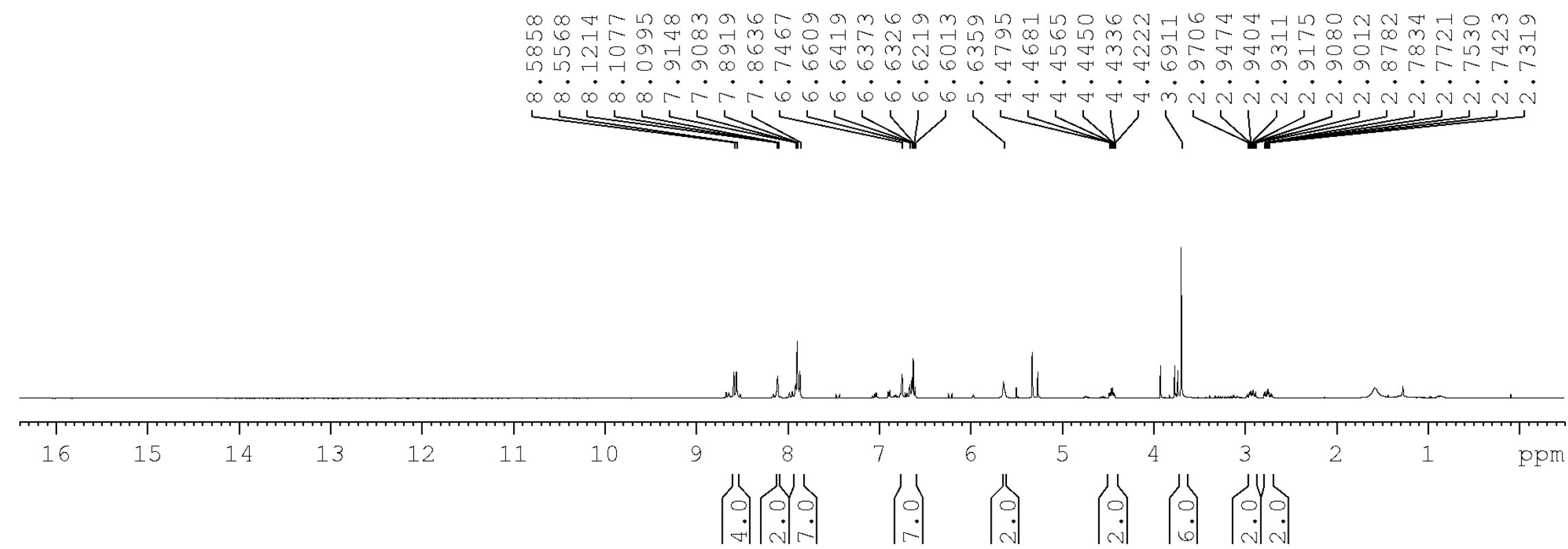

Figure S37. ${ }^{1} \mathrm{H}$ NMR spectrum of mono-2ae and meso-3ae' 

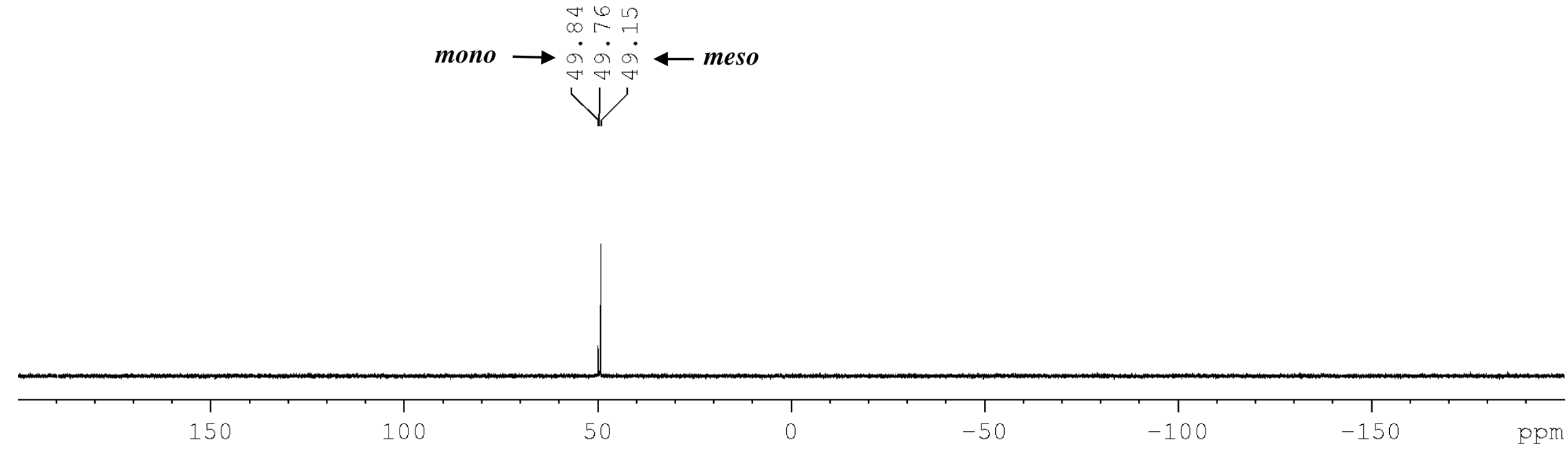

Figure S38. ${ }^{31} \mathrm{P}\left\{{ }^{1} \mathrm{H}\right\} \mathrm{NMR}$ spectrum of mono-2ae and meso-3ae' 


\section{C11 Complex rac-4aa}

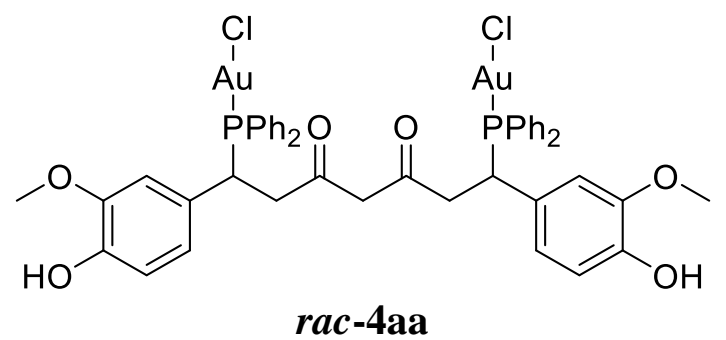

${ }^{1}$ H NMR spectrum

$\left(400 \mathrm{MHz}, \mathrm{CD}_{2} \mathrm{Cl}_{2}\right.$ )

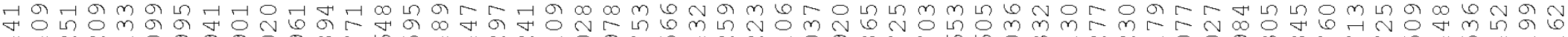

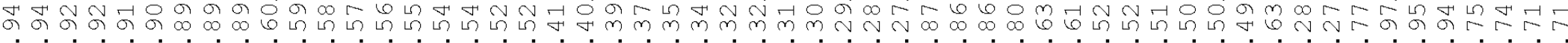

. . - .

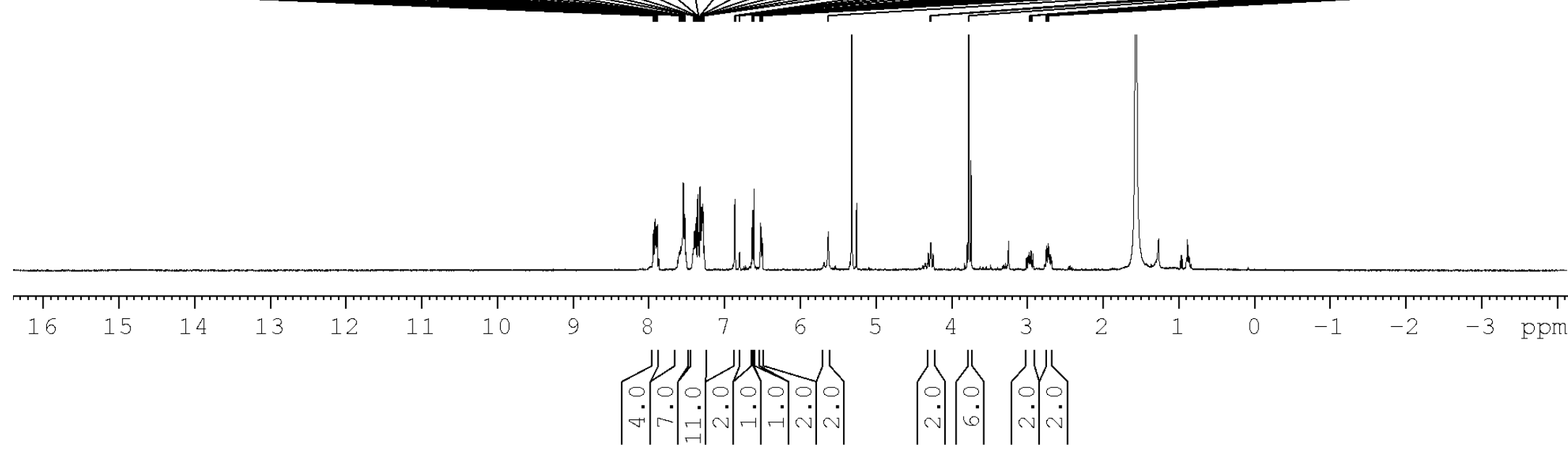

Figure S39. ${ }^{1} \mathrm{H}$ NMR spectrum of $r a c-4 a a$ 


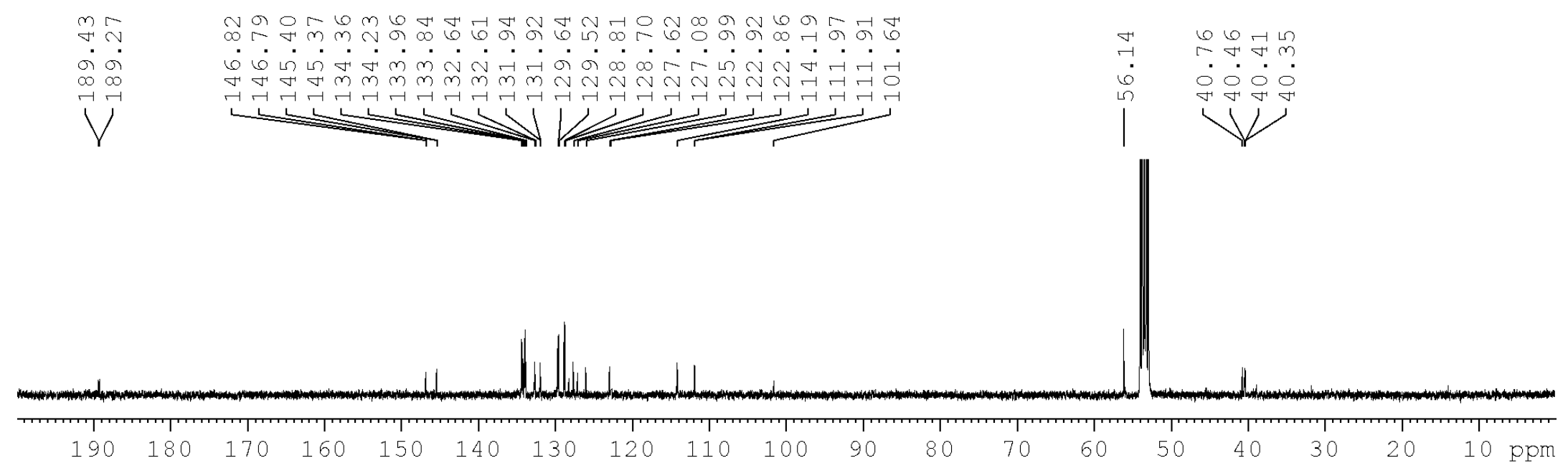

Figure S40. ${ }^{13} \mathrm{C}$ NMR spectrum of $r a c-4 a a$ 


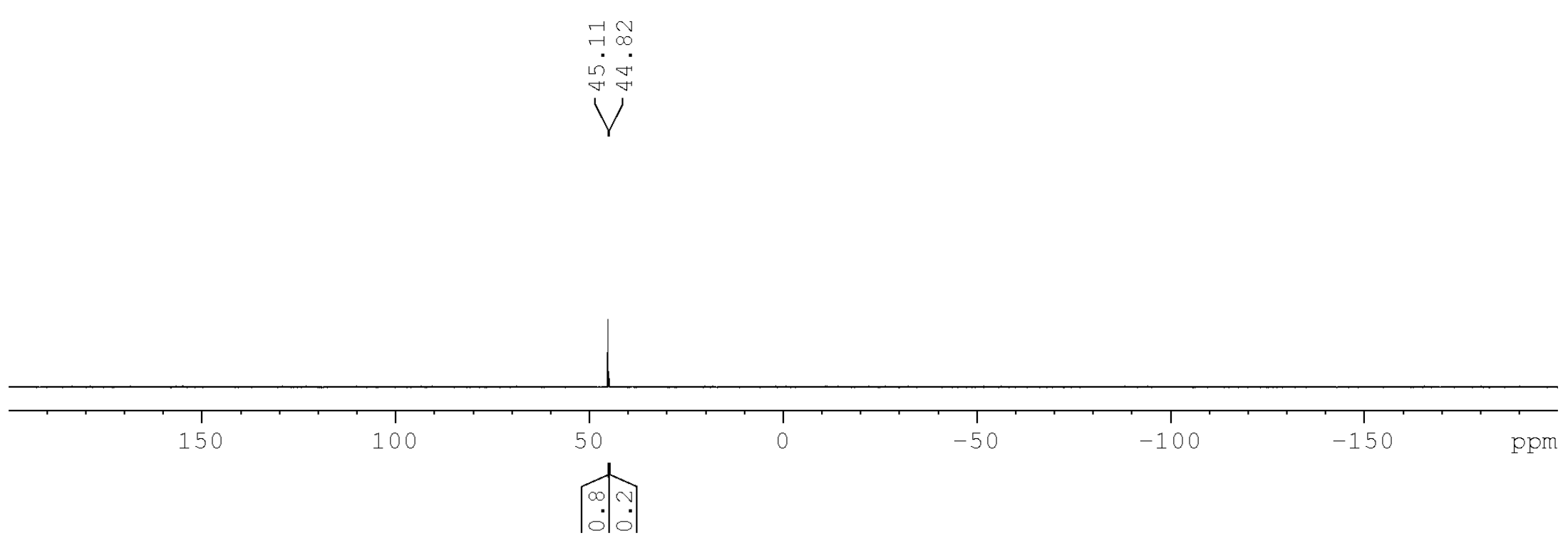

Figure S41. ${ }^{31} \mathrm{P}\left\{{ }^{1} \mathrm{H}\right\}$ NMR spectrum of rac-4aa 


\section{C12 Complex meso-4aa'}

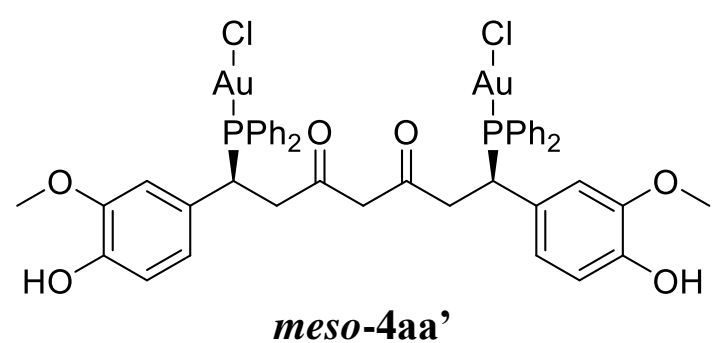

${ }^{1}$ H NMR spectrum

(400 MHz, $\mathrm{CD}_{2} \mathrm{Cl}_{2}$ )

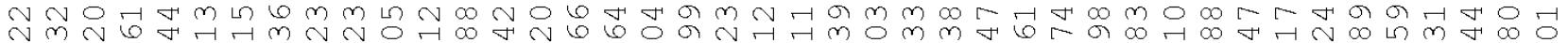
ڤ̆ -
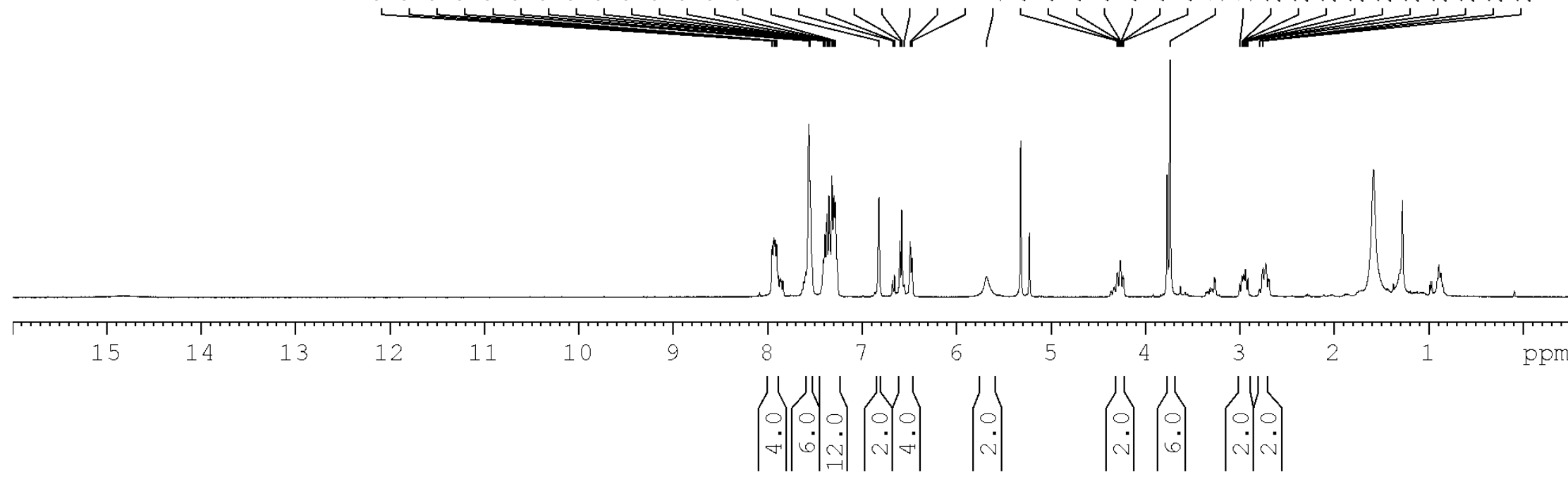

Figure S42. ${ }^{31} \mathrm{P}\left\{{ }^{1} \mathrm{H}\right\}$ NMR spectrum of meso-4aa' 


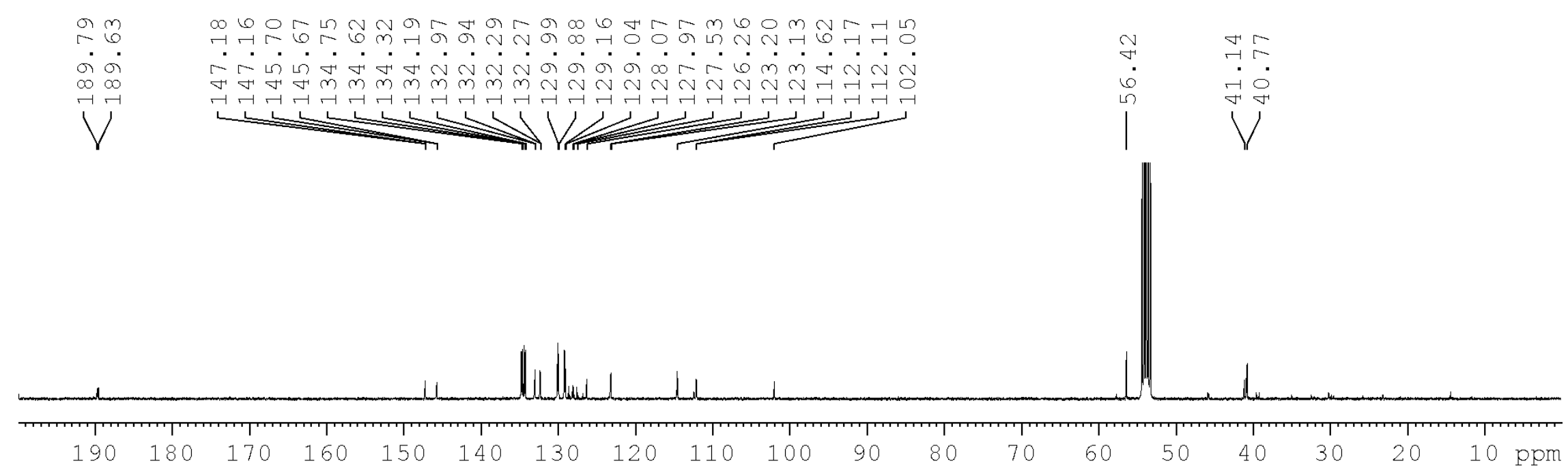

Figure S43. ${ }^{13} \mathrm{C}$ NMR spectrum of meso-4aa' 


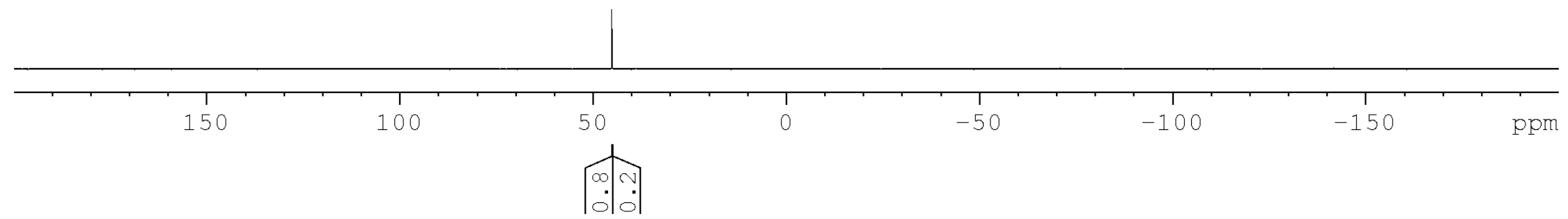

Figure S44. ${ }^{31} \mathrm{P}\left\{{ }^{1} \mathrm{H}\right\}$ NMR spectrum of meso-4aa' 


\section{DIASTEREOMERIC RATIO AND ENANTIOMERIC EXCESS DATA}

D1 Diastereomeric Ratio of chiral-3aa and meso-3aa'

Curcumin + Diphenylphosphine at $25{ }^{\circ} \mathrm{C}$
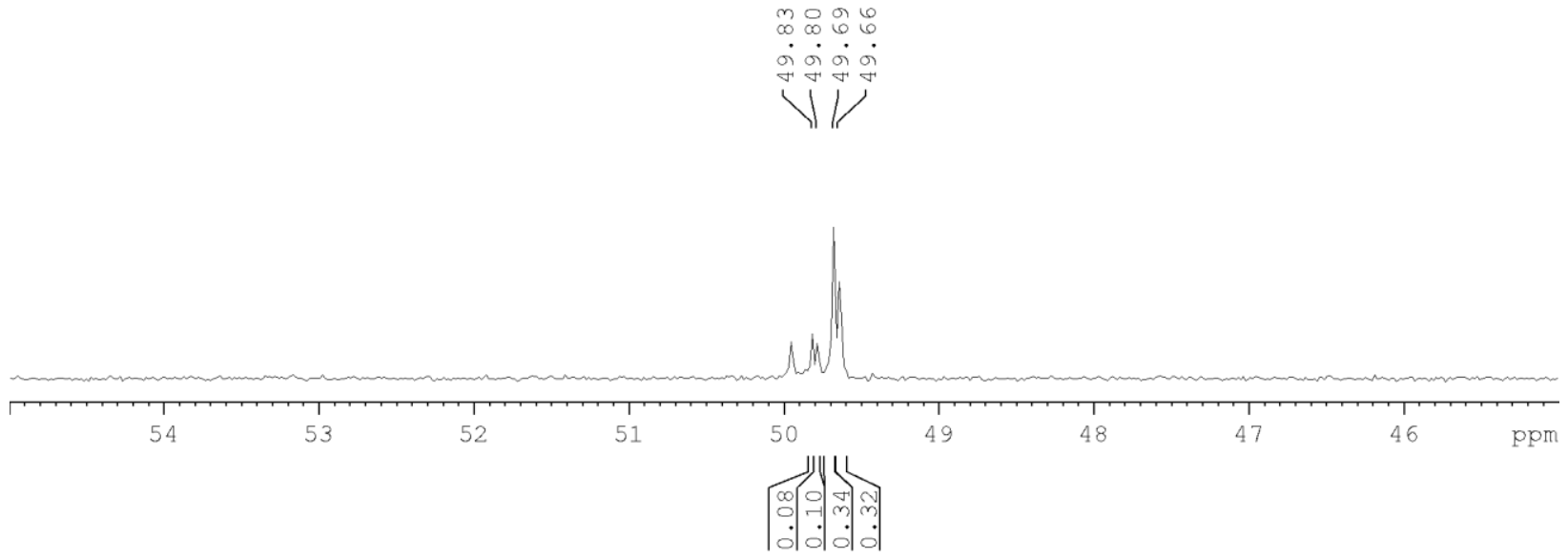

Figure S45. ${ }^{31} \mathrm{P}\left\{{ }^{1} \mathrm{H}\right\}$ NMR spectrum of chiral-3aa and meso-3aa', reaction at $25{ }^{\circ} \mathrm{C}$

Curcumin + Diphenylphosphine at $0{ }^{\circ} \mathrm{C}$

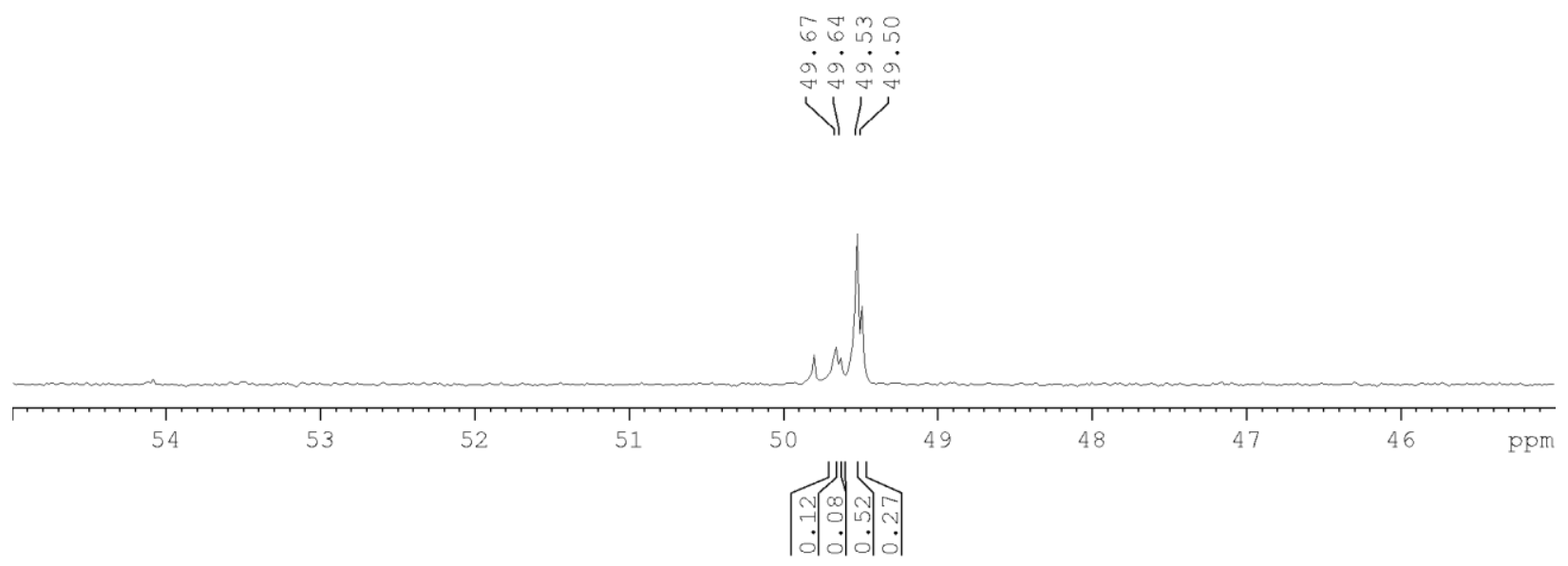

Figure S46. ${ }^{31} \mathrm{P}\left\{{ }^{1} \mathrm{H}\right\}$ NMR spectrum of chiral-3aa and meso-3aa', reaction at $0{ }^{\circ} \mathrm{C}$ 
D2 Diastereomeric Ratio and Enantiomeric Excess

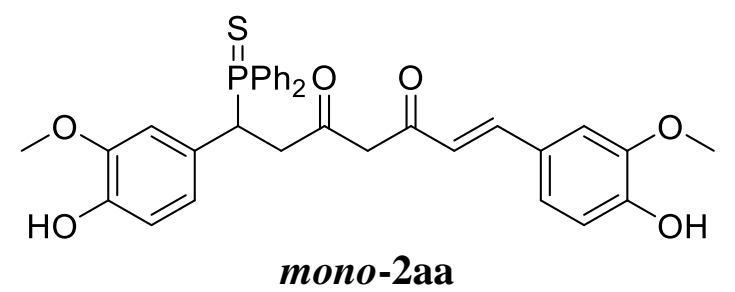

\section{HPLC Conditions}

Column:

Diacel Chiralpak ${ }^{\circledR}$ ID column

Eluent:

Hexane $/{ }^{i} \mathrm{PrOH}$ (75:25)

Flow Rate:

$1 \mathrm{~mL} / \mathrm{min}$

Column Temperature: $\quad 30{ }^{\circ} \mathrm{C}$

Wavelength:

$210 \mathrm{~nm}$

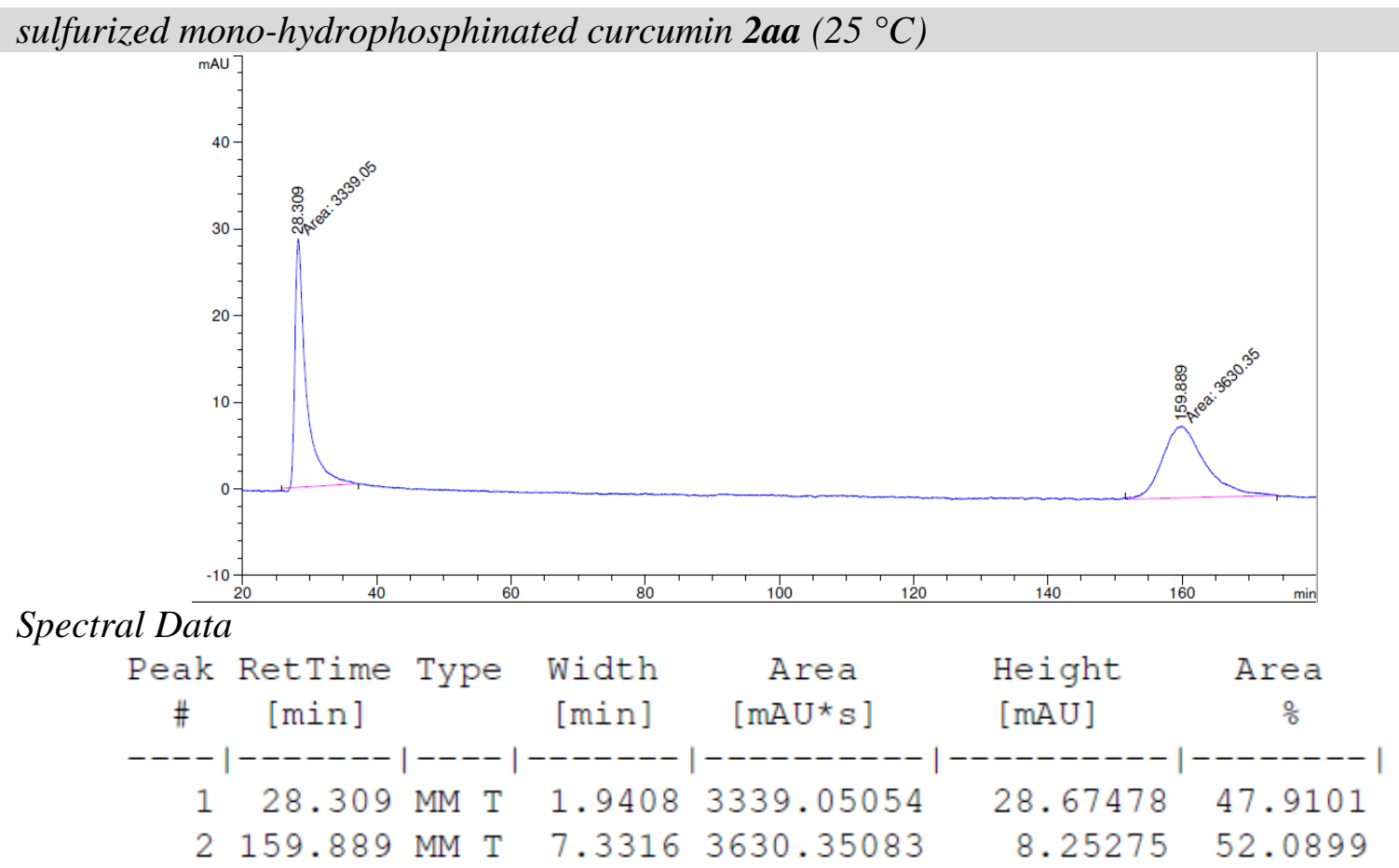

Figure S47. Chiral HPLC spectrum of racemic mono-2aa 


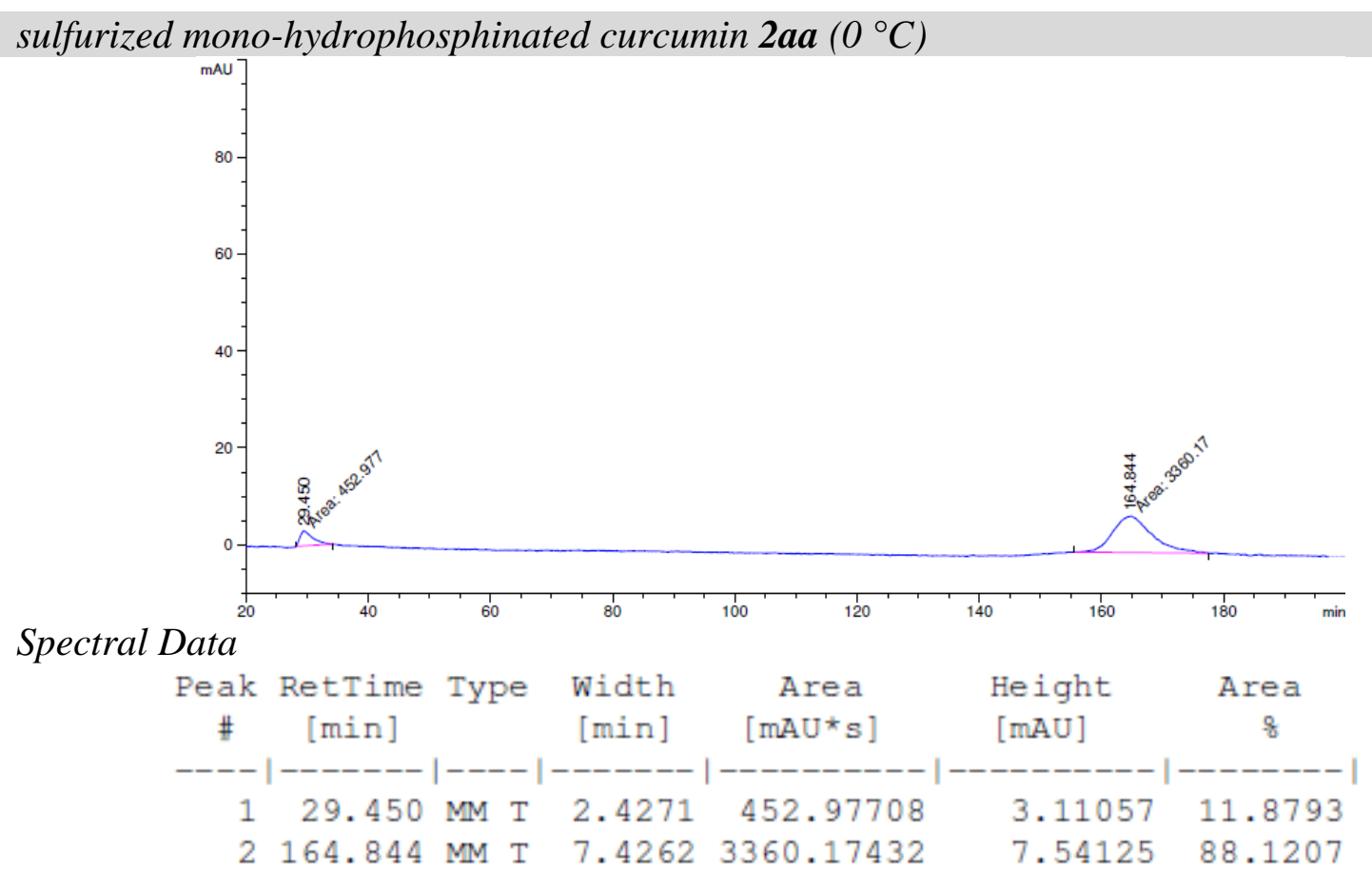

Figure S48. Chiral HPLC spectrum of enantioenriched mono-2aa 


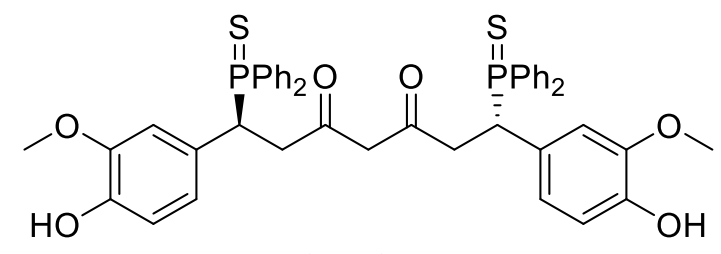

chiral-3aa

\section{HPLC Conditions}

Column:

Eluent:

Flow Rate:

Column Temperature:

Wavelength:

Diacel Chiralpak ${ }^{\circledR}$ ID column

Hexane $/{ }^{i} \mathrm{PrOH}(75: 25)$

$1 \mathrm{~mL} / \mathrm{min}$

$30{ }^{\circ} \mathrm{C}$

$210 \mathrm{~nm}$

racemic sulfurized di-hydrophosphinated curcumin 3aa

Spectral Data
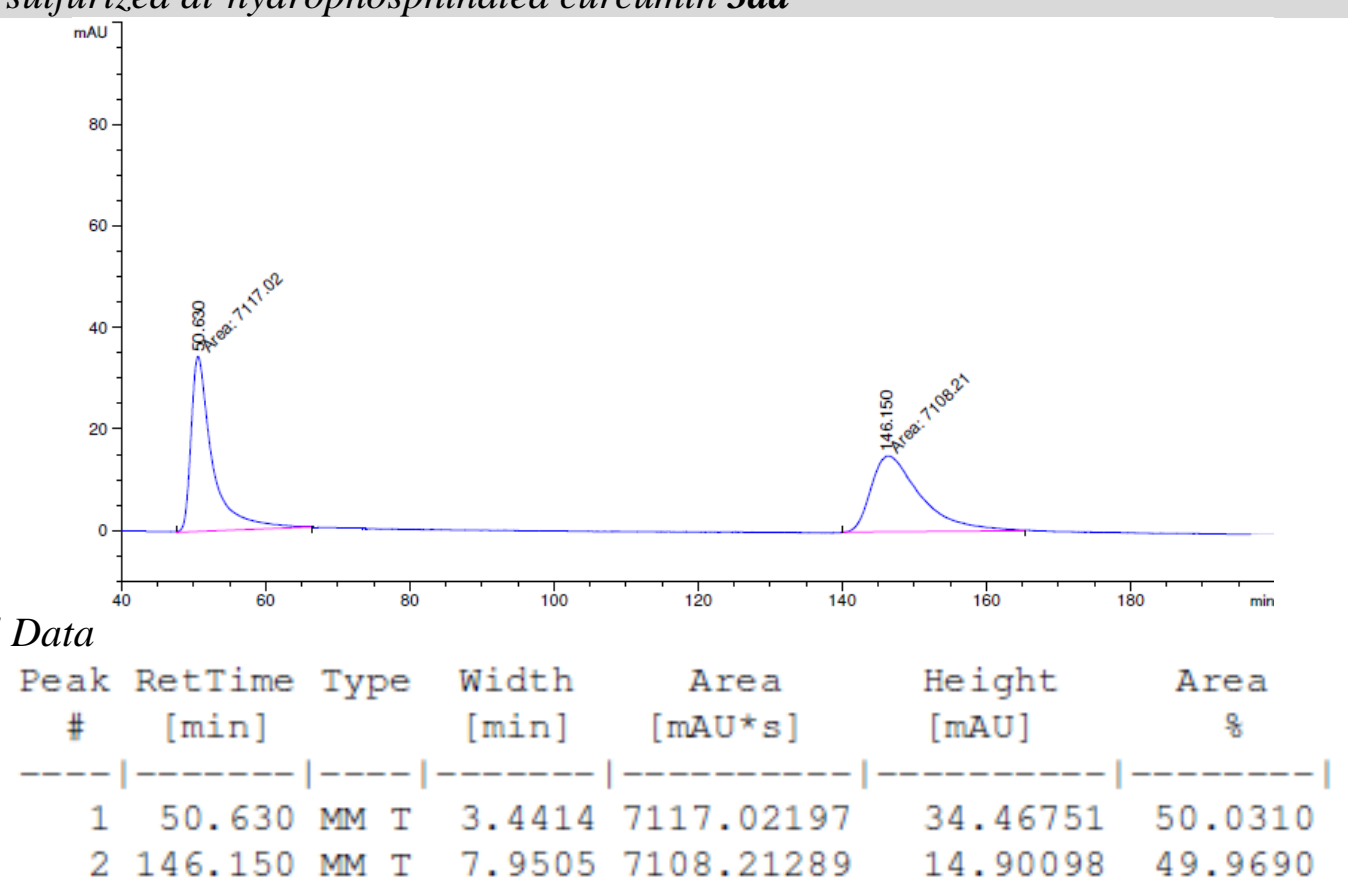

Figure S49. Chiral HPLC spectrum of racemic chiral-3aa 


\section{chiral sulfurized di-hydrophosphinated curcumin $3 a a$}

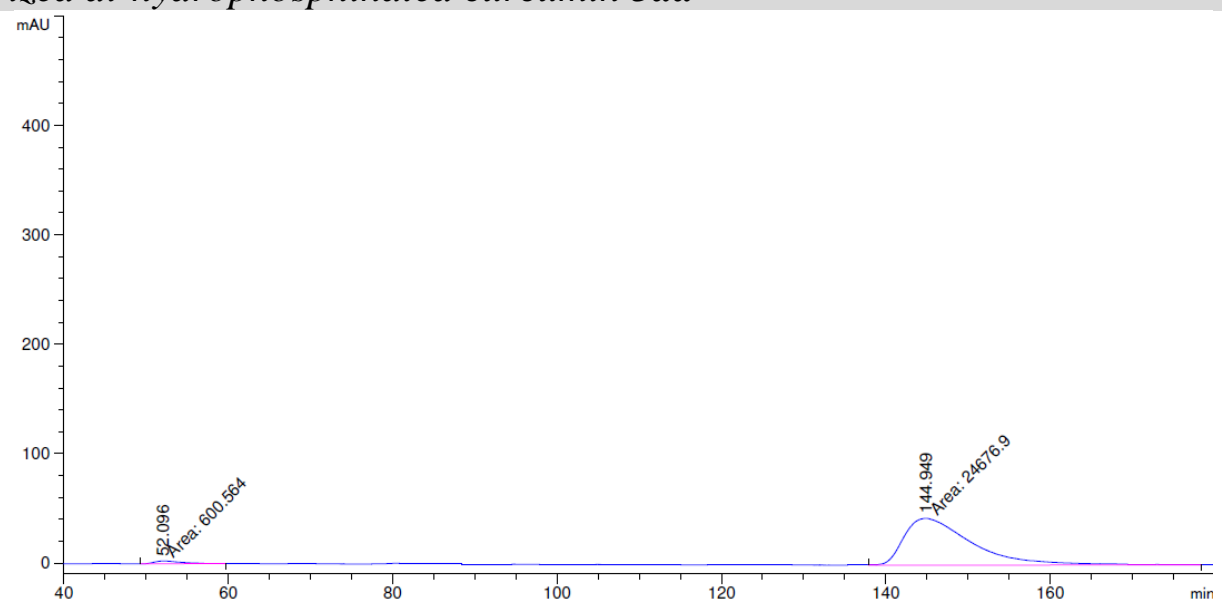

Spectral Data

\begin{tabular}{|c|c|c|c|c|c|c|}
\hline $\begin{array}{c}\text { Peak } \\
\#\end{array}$ & $\begin{array}{c}\text { RetTime } \\
\text { [min] }\end{array}$ & Type & $\begin{array}{c}\text { Width } \\
\text { [min] }\end{array}$ & $\begin{array}{c}\text { Area } \\
{\left[\mathrm{mAU}^{\star} \mathrm{s}\right]}\end{array}$ & $\begin{array}{l}\text { Height } \\
{[\mathrm{mAU}]}\end{array}$ & $\begin{array}{c}\text { Area } \\
\frac{\circ}{\delta}\end{array}$ \\
\hline 1 & 096 & MM T & 103 & 600.56421 & 812 & 759 \\
\hline 2 & 144.949 & MM T & 9.5815 & $.46769 \mathrm{e} 4$ & 42.92478 & 97.6241 \\
\hline
\end{tabular}

Figure S50. Chiral HPLC spectrum of enantioenriched chiral-3aa 
<smiles>O=C(CC(=O)CC(c1ccc(O)cc1)c1ccc(O)cc1)CC(=P)[PH](P)=S</smiles>

3ba/3ba' (reported as an inseparable diastereomeric mixture)

$\begin{array}{ll}\text { HPLC Conditions } & \\ \text { Column: } & \text { Diacel Chiralpak }{ }^{\circledR} \mathrm{IC} \text { column } \\ \text { Eluent: } & \text { Hexane } /{ }^{i} \mathrm{PrOH}(85: 15) \\ \text { Flow Rate: } & 1 \mathrm{~mL} / \mathrm{min} \\ \text { Column Temperature: } & 35^{\circ} \mathrm{C} \\ \text { Wavelength: } & 210 \mathrm{~nm}\end{array}$

racemic sulfurized di-hydrophosphinated bisdemethoxycurcumin $3 \mathbf{b a}$ and meso-3ba'

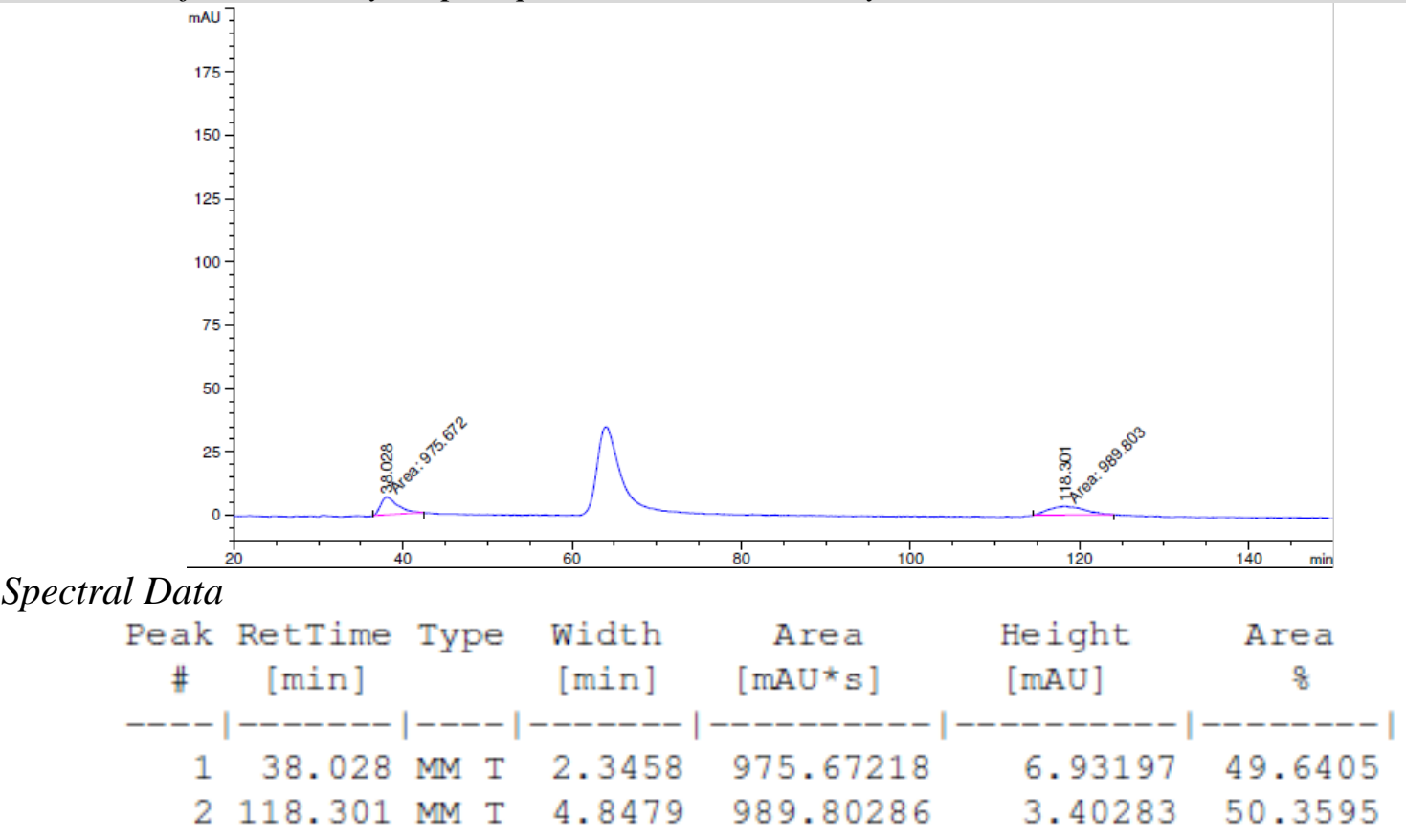

Figure S51. Chiral HPLC spectrum of racemic 3ba/3ba' 


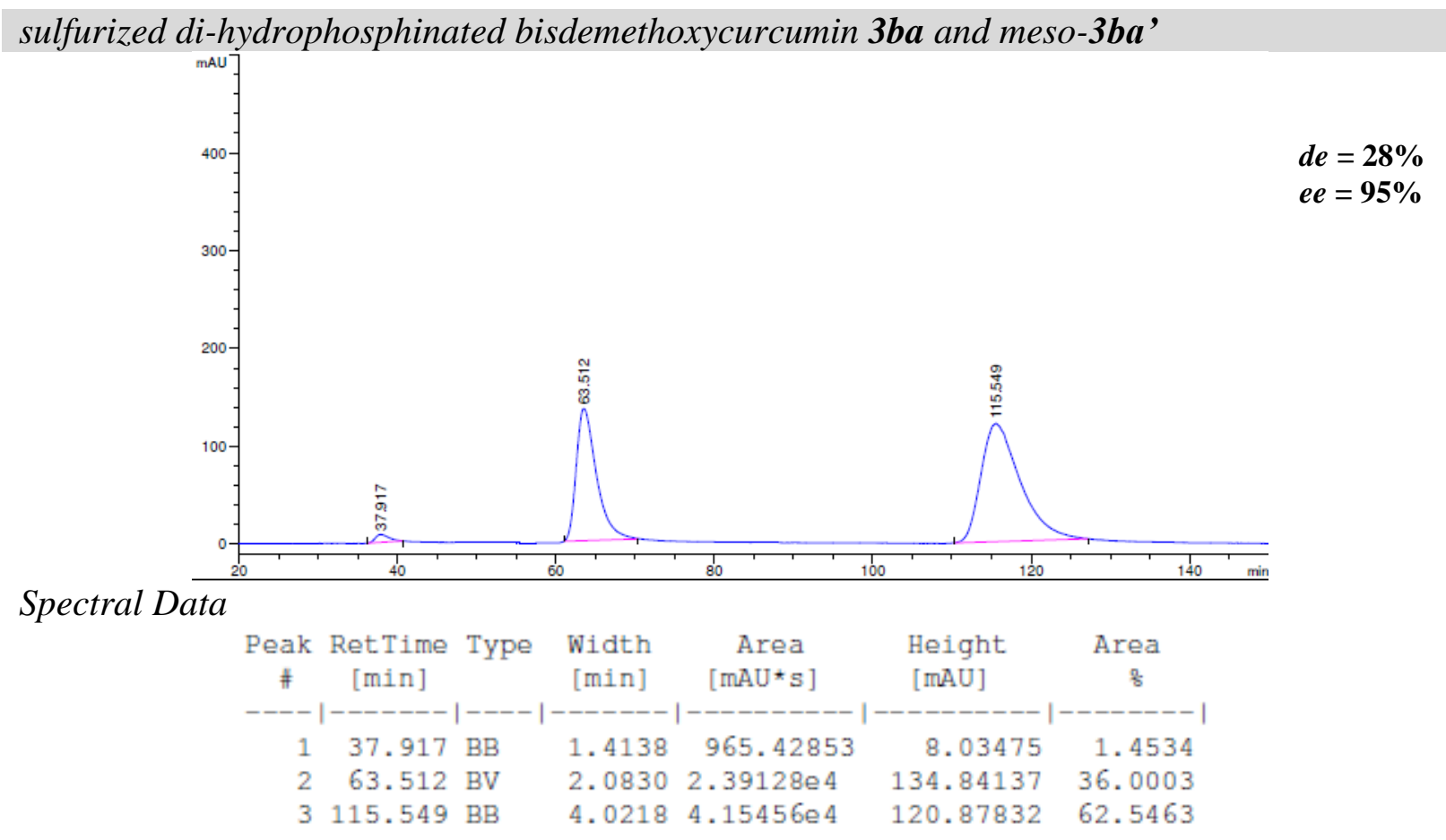

Figure S52. Chiral HPLC spectrum of enantioenriched 3ba/3ba' 


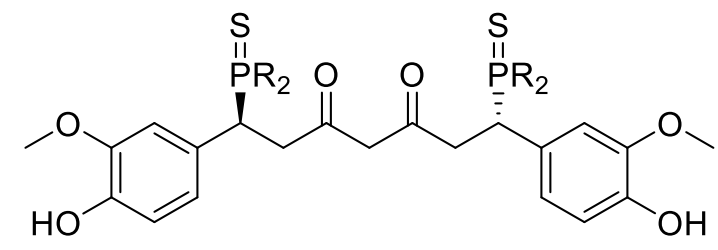

3ab

$\begin{array}{ll}\text { HPLC Conditions } & \\ \text { Column: } & \text { Diacel Chiralpak }{ }^{\circledR} \mathrm{IF} \text { column } \\ \text { Eluent: } & \text { Hexane } / \text { PrOH }(75: 25) \\ \text { Flow Rate: } & 1 \mathrm{~mL} / \mathrm{min} \\ \text { Column Temperature: } & 30{ }^{\circ} \mathrm{C} \\ \text { Wavelength: } & 250 \mathrm{~nm}\end{array}$

racemic sulfurized di-hydrophosphinated $\mathbf{3 a b}$

Spectral Data

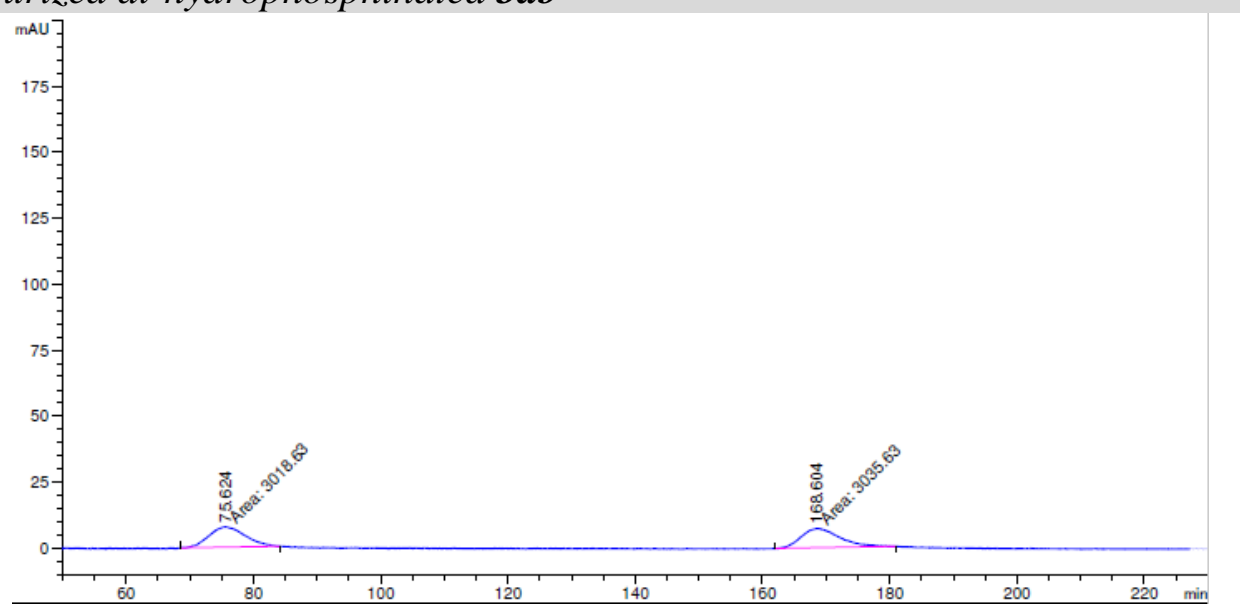

\begin{tabular}{|c|c|c|c|c|c|c|}
\hline $\begin{array}{c}\text { Peak } \\
\#\end{array}$ & $\begin{array}{l}\text { RetTime } \\
\text { [min] }\end{array}$ & Type & $\begin{array}{l}\text { Width } \\
\text { [min] }\end{array}$ & $\begin{array}{c}\text { Area } \\
{[\mathrm{mAU} * \mathrm{~s}]}\end{array}$ & $\begin{array}{l}\text { Height } \\
\text { [mAU] }\end{array}$ & $\begin{array}{c}\text { Area } \\
\frac{8}{8}\end{array}$ \\
\hline 1 & & & & 3018.62891 & & \\
\hline 2 & 168.604 & $\mathrm{MM} \mathrm{T}$ & 6.9258 & 3035.62573 & 7. 30517 & 50.1404 \\
\hline
\end{tabular}

Figure S53. Chiral HPLC spectrum of racemic 3ab 
chiral sulfurized di-hydrophosphinated $\mathbf{3 a b}$

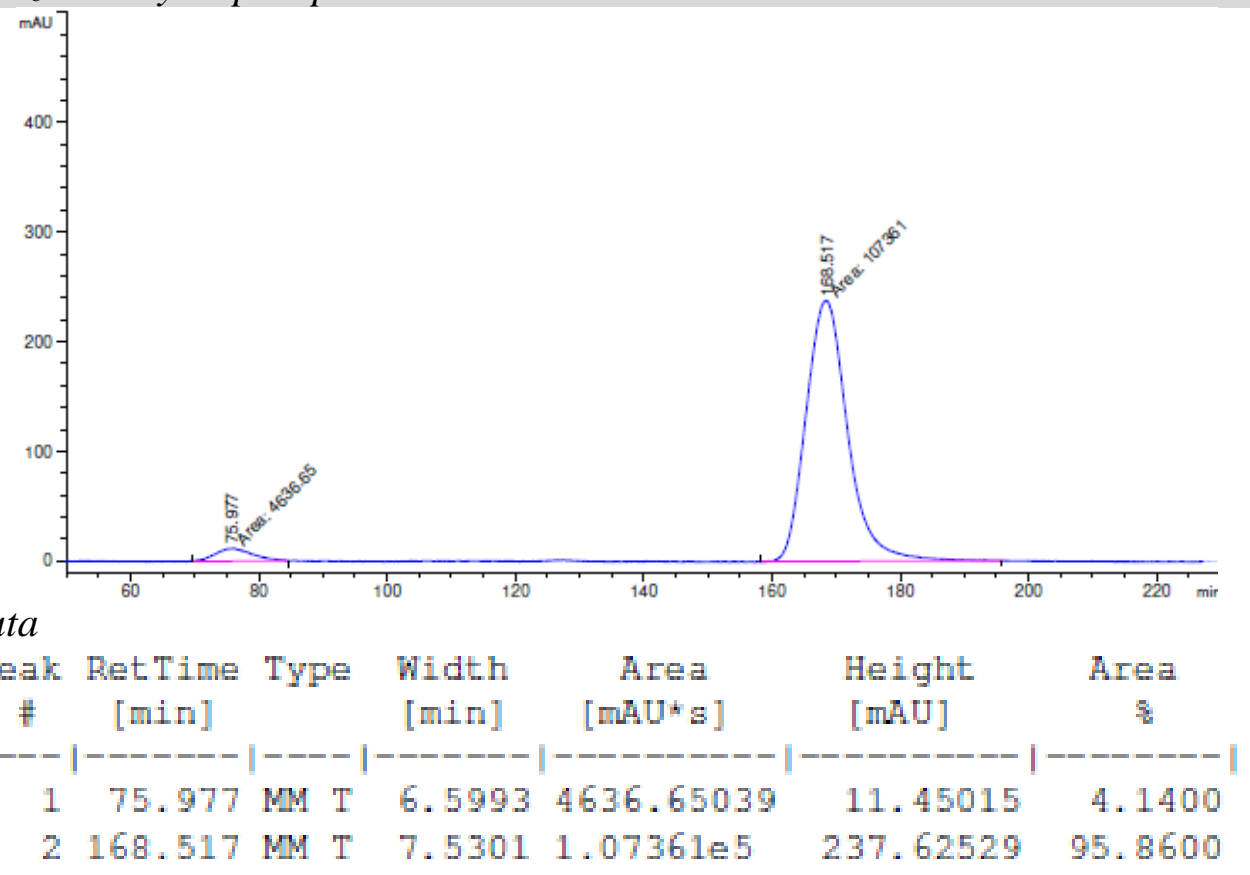

Figure S54. Chiral HPLC spectrum of enantioenriched 3ab 
<smiles>[R2][C@H](CC(=O)CC(=O)CC([PH]=S)c1ccc(O)c(OC)c1)c1ccc(O)c(OC)c1</smiles>

3ac/3ac' (reported as an inseparable diastereomeric mixture)

\section{HPLC Conditions}

Column:

Eluent:

Flow Rate:

Column Temperature:

Wavelength:
Diacel Chiralpak ${ }^{\circledR}$ IA column

Hexane $/{ }^{i} \mathrm{PrOH}(85: 15)$

$1 \mathrm{~mL} / \mathrm{min}$

$35^{\circ} \mathrm{C}$

$210 \mathrm{~nm}$

racemic sulfurized di-hydrophosphinated curcumin $3 a c$ and meso-3ac'

Spectral Data
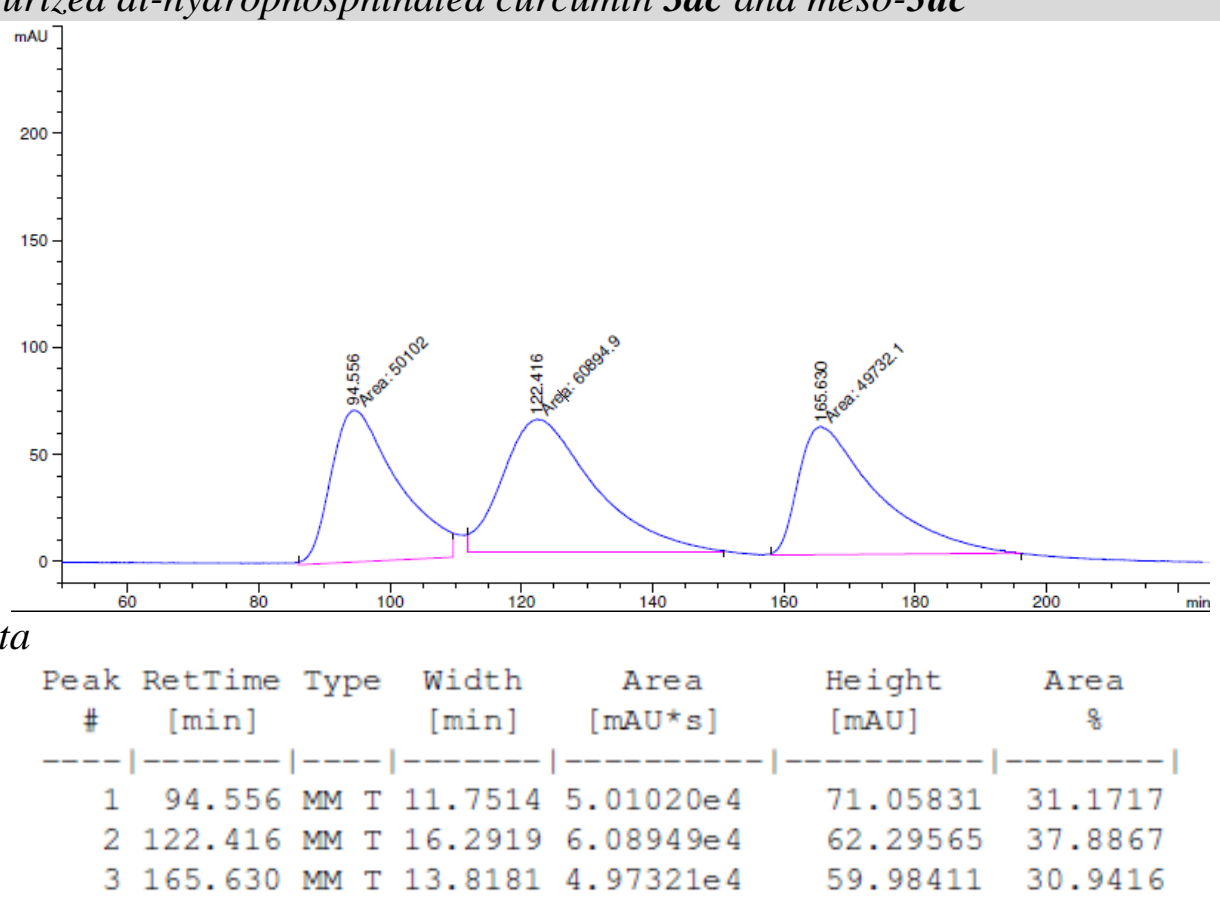

Figure S55. Chiral HPLC spectrum of racemic 3ac/3ac' 


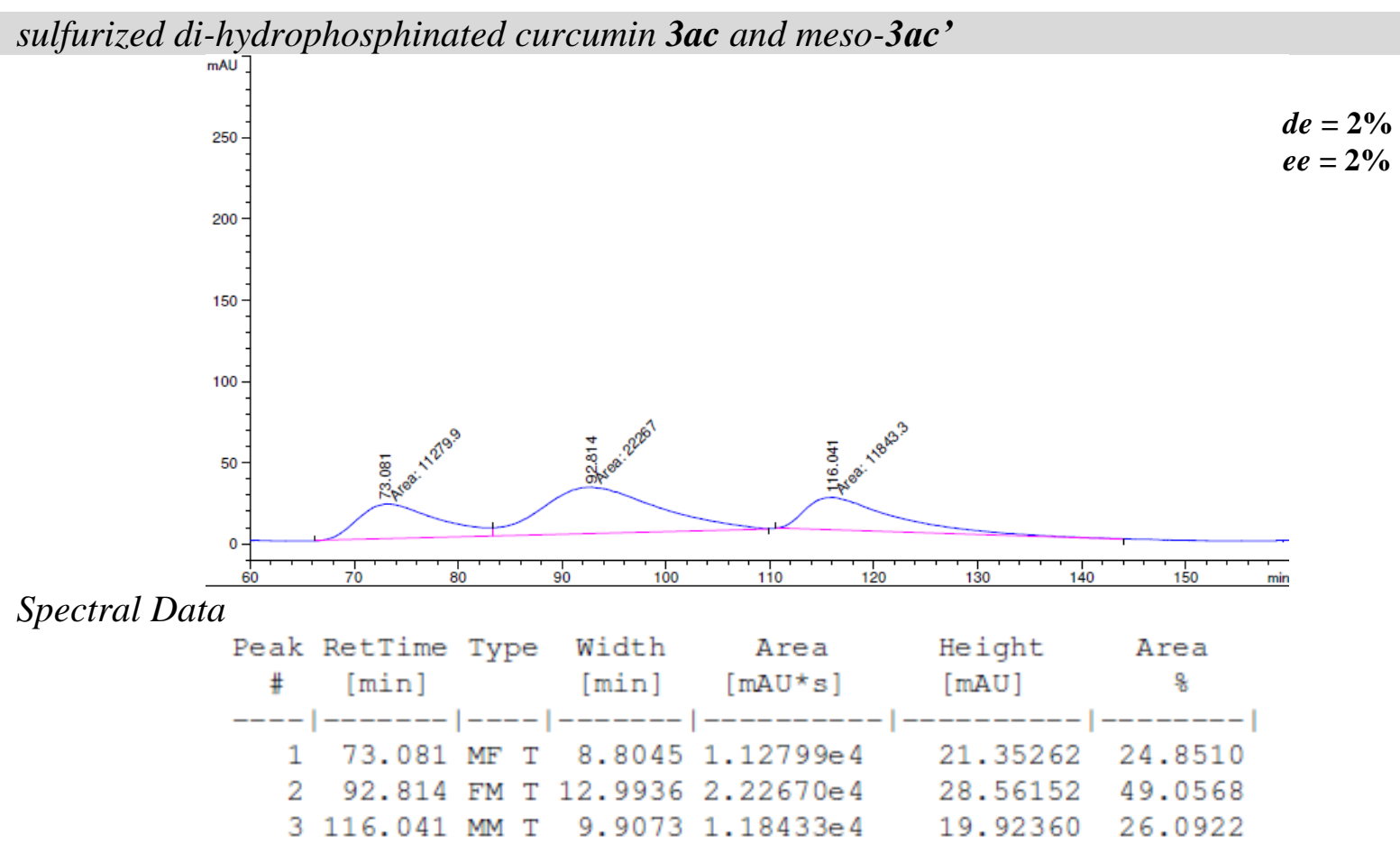

Figure S56. Chiral HPLC spectrum of enantioenriched 3ac/3ac' 
<smiles>[R2]PC(CC(=O)CC(=O)CC(P=S)c1ccc(O)c(OC)c1)c1ccc(O)c(OC)c1</smiles>

3ad/3ad' (reported as an inseparable diastereomeric mixture)

$\begin{array}{ll}\text { HPLC Conditions } & \\ \text { Column: } & \text { Diacel Chiralpak }{ }^{\circledR} \mathrm{ID} \text { column } \\ \text { Eluent: } & \text { Hexane/PrOH }(75: 25) \\ \text { Flow Rate: } & 1 \mathrm{~mL} / \mathrm{min} \\ \text { Column Temperature: } & 30{ }^{\circ} \mathrm{C} \\ \text { Wavelength: } & 230 \mathrm{~nm}\end{array}$

racemic sulfurized di-hydrophosphinated curcumin 3ad and meso-3ad'

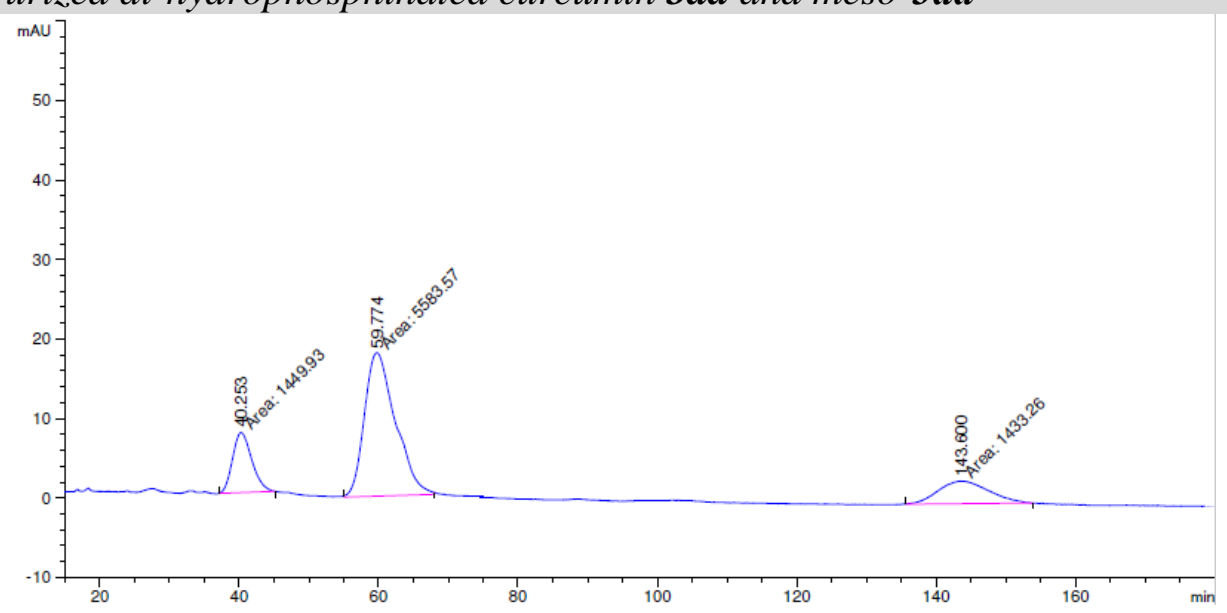

Spectral Data

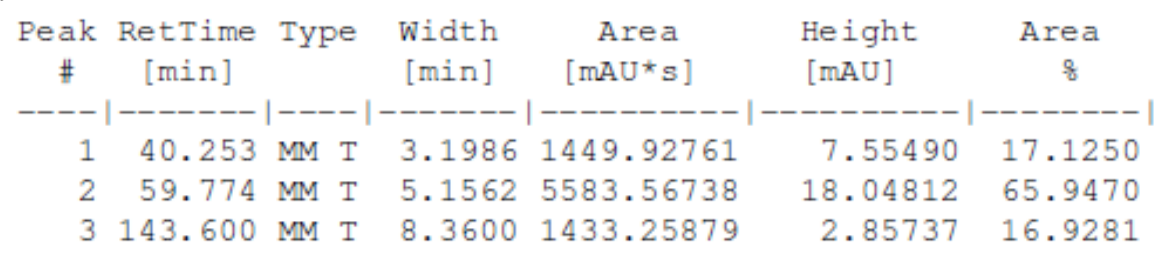

Figure S57. Chiral HPLC spectrum of racemic 3ad/3ad' 


\section{HPLC Conditions}

Column:

Eluent:

Flow Rate:

Column Temperature:

Wavelength:
Diacel Chiralpak ${ }^{\circledR}$ ID column

Hexane $/{ }^{i} \mathrm{PrOH}(75: 25)$

$1 \mathrm{~mL} / \mathrm{min}$

$30{ }^{\circ} \mathrm{C}$

$230 \mathrm{~nm}$

sulfurized di-hydrophosphinated curcumin chiral-3ad and meso-3ad'

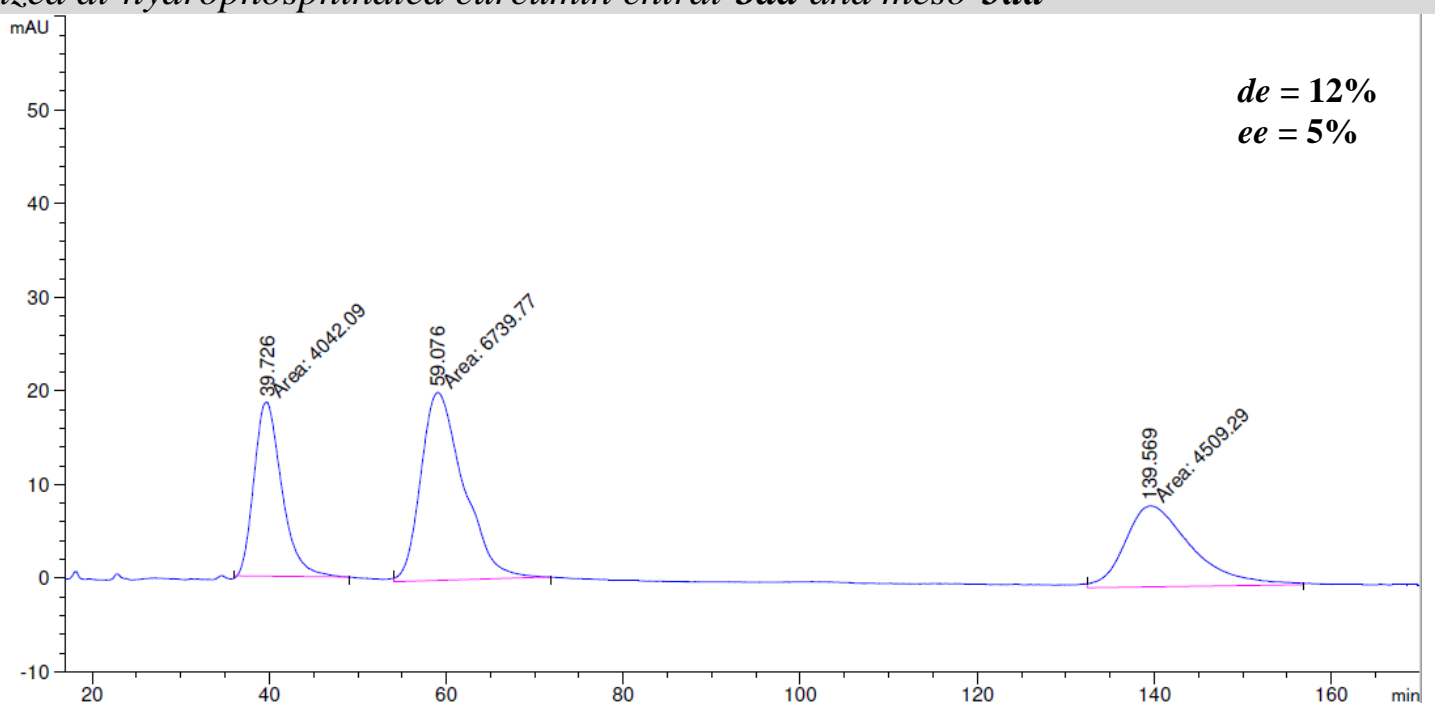

Spectral Data

\begin{tabular}{|c|c|c|c|c|c|c|}
\hline $\begin{array}{c}\text { Peak } \\
\#\end{array}$ & $\begin{array}{c}\text { RetTime } \\
\text { [min] }\end{array}$ & Type & $\begin{array}{c}\text { Width } \\
\text { [min] }\end{array}$ & $\begin{array}{c}\text { Area } \\
{\left[\mathrm{mAU}^{*} \mathrm{~s}\right]}\end{array}$ & $\begin{array}{l}\text { Height } \\
{[\mathrm{mAU}]}\end{array}$ & $\begin{array}{c}\text { Area } \\
\frac{\circ}{\delta}\end{array}$ \\
\hline & & & & 5 & & \\
\hline 1 & 39.726 & MM T & 3.6260 & 4042.08765 & 18.57934 & 26.4342 \\
\hline 2 & 59.076 & MM T & 02 & 6739.76563 & 5820 & 763 \\
\hline 3 & 139.569 & MM T & & 4509.28564 & 2202 & 4895 \\
\hline
\end{tabular}

Figure S58. Chiral HPLC spectrum of enantioenriched 3ad/3ad' 


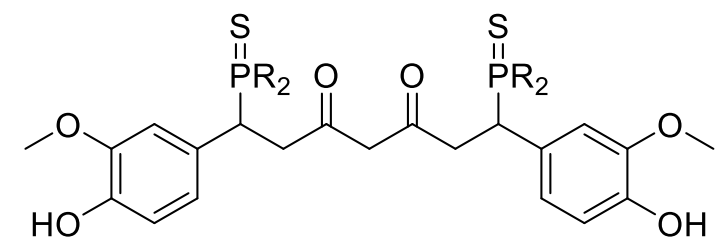

3ae

\section{HPLC Conditions}

Column:

Eluent:

Flow Rate:

Diacel Chiralpak ${ }^{\circledR}$ IB column

Hexane/ ${ }^{i} \mathrm{PrOH}$ (97:3)

Column Temperature:

$1 \mathrm{~mL} / \mathrm{min}$

Wavelength:

$30{ }^{\circ} \mathrm{C}$

$250 \mathrm{~nm}$

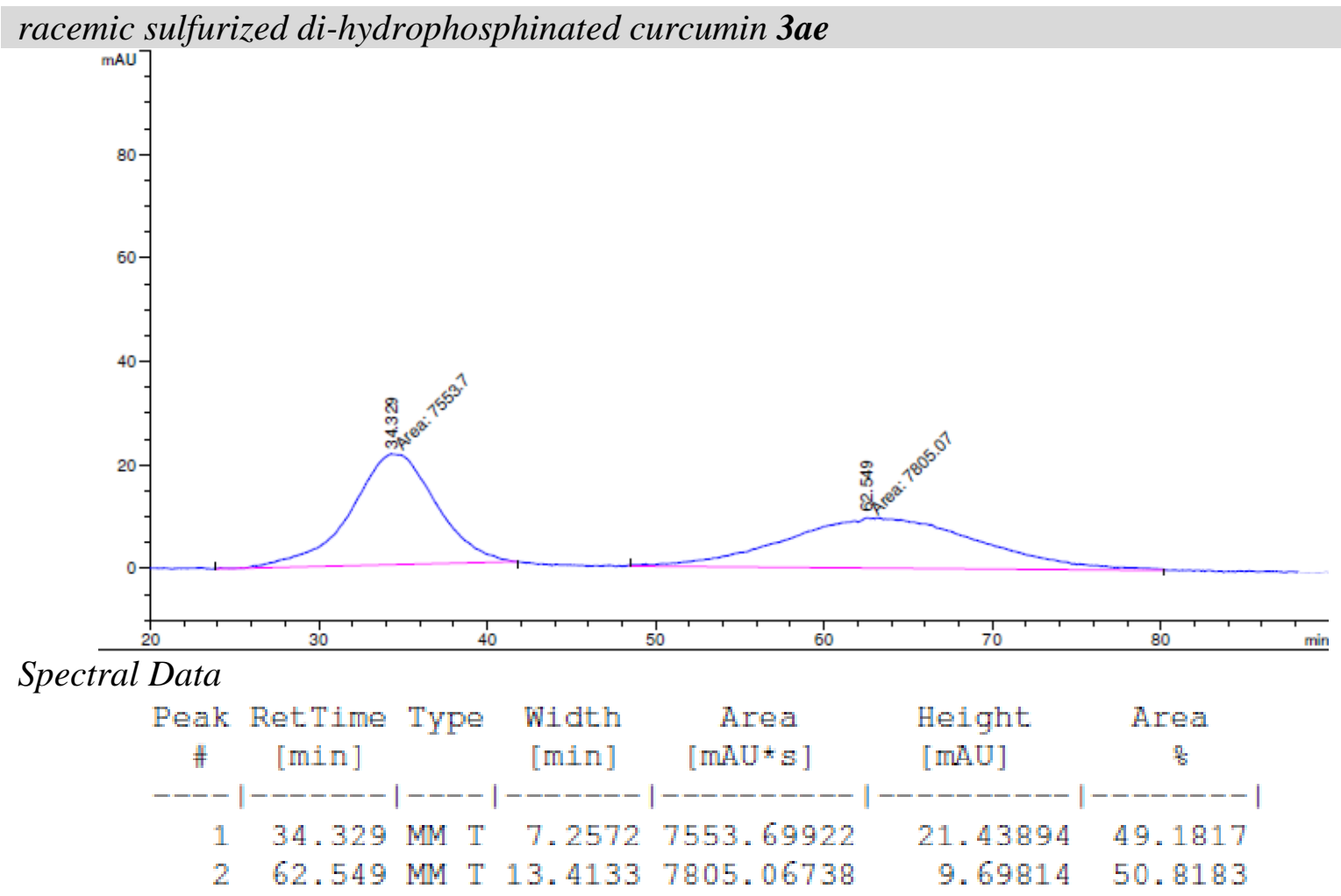

Figure S59. Chiral HPLC spectrum of racemic 3ae 


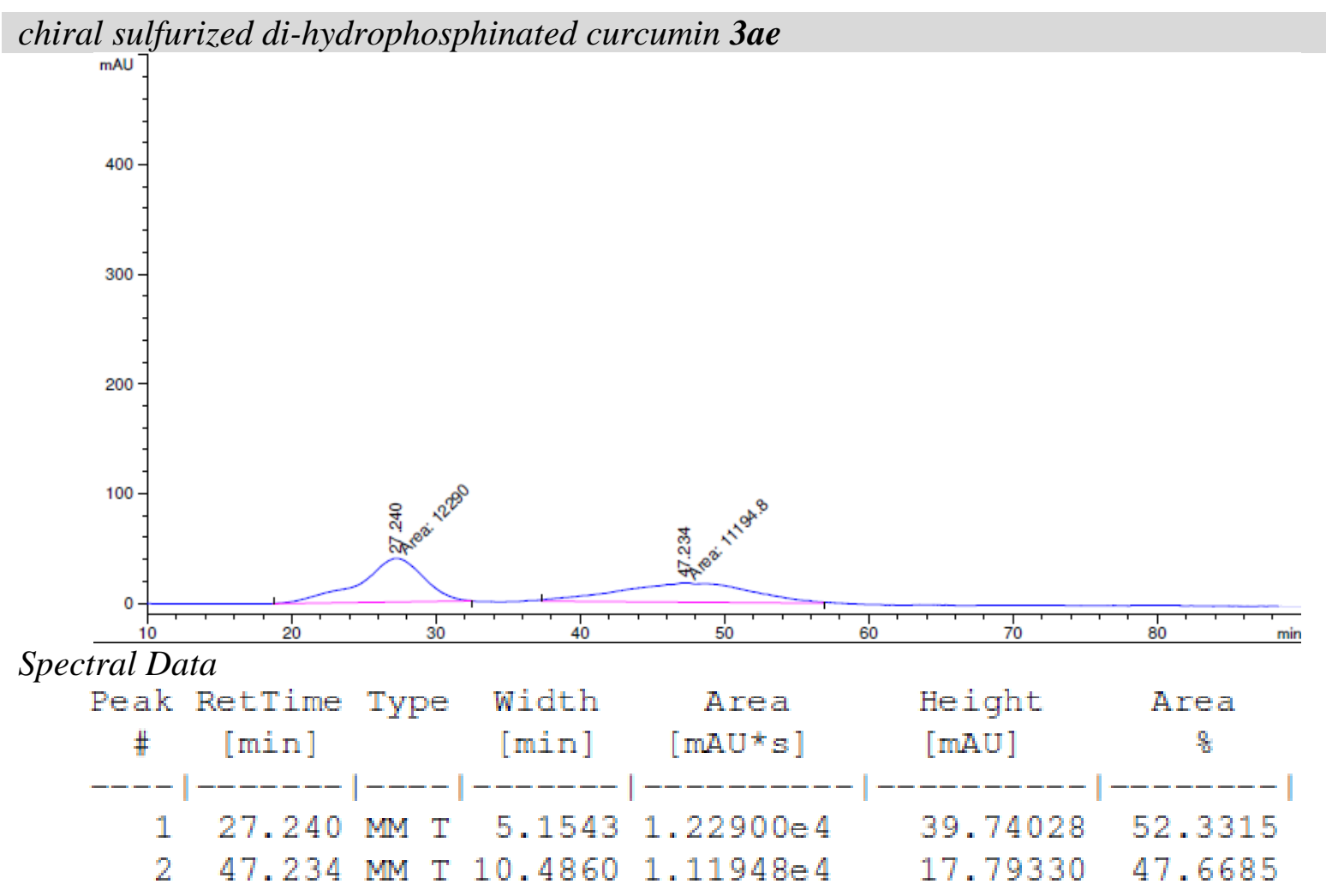

Figure S60. Chiral HPLC spectrum of enantioenriched 3ae 


\section{E DETECTION OF CHARACTERISTIC PROTON SIGNAL OF ENOL IN DI- HYDROPHOSPHINATED PRODUCT}

\section{E1 Evidence of Keto-Enol Equilibrium}

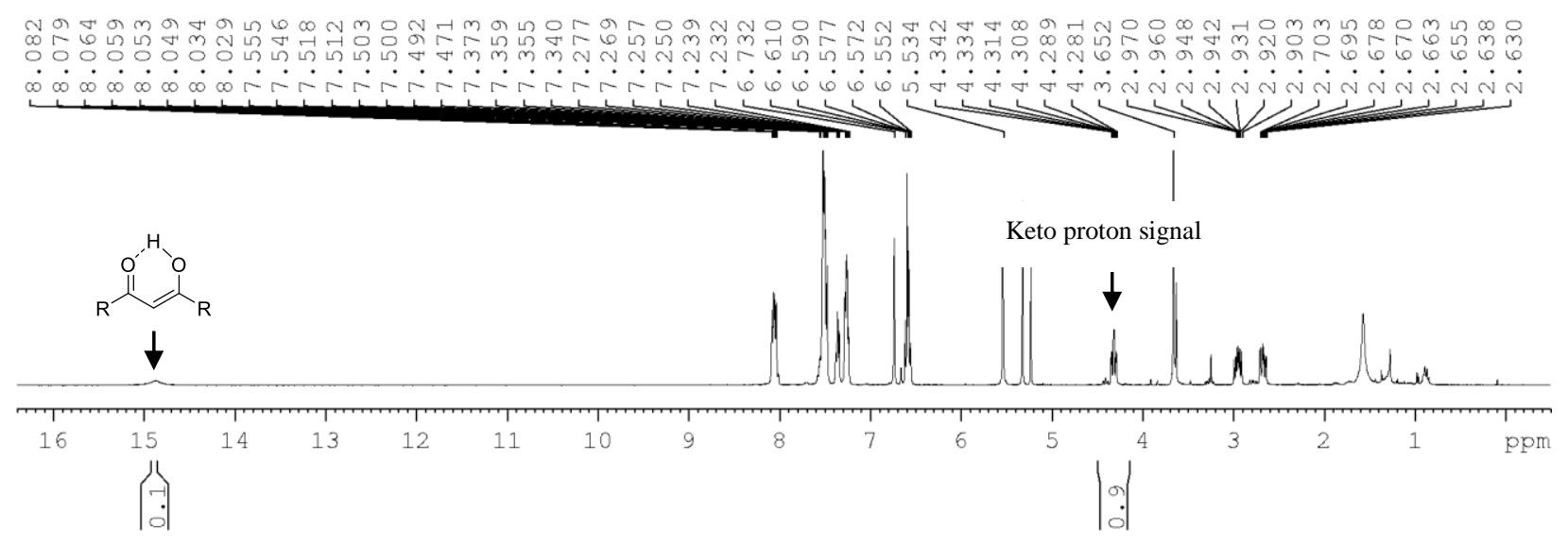

Figure S61. ${ }^{1} \mathrm{H}$ NMR spectrum of dihydrophosphinated product 


\section{F PROPOSED REACTION PATHWAY}

F1 Proposed Mechanistic Cycle
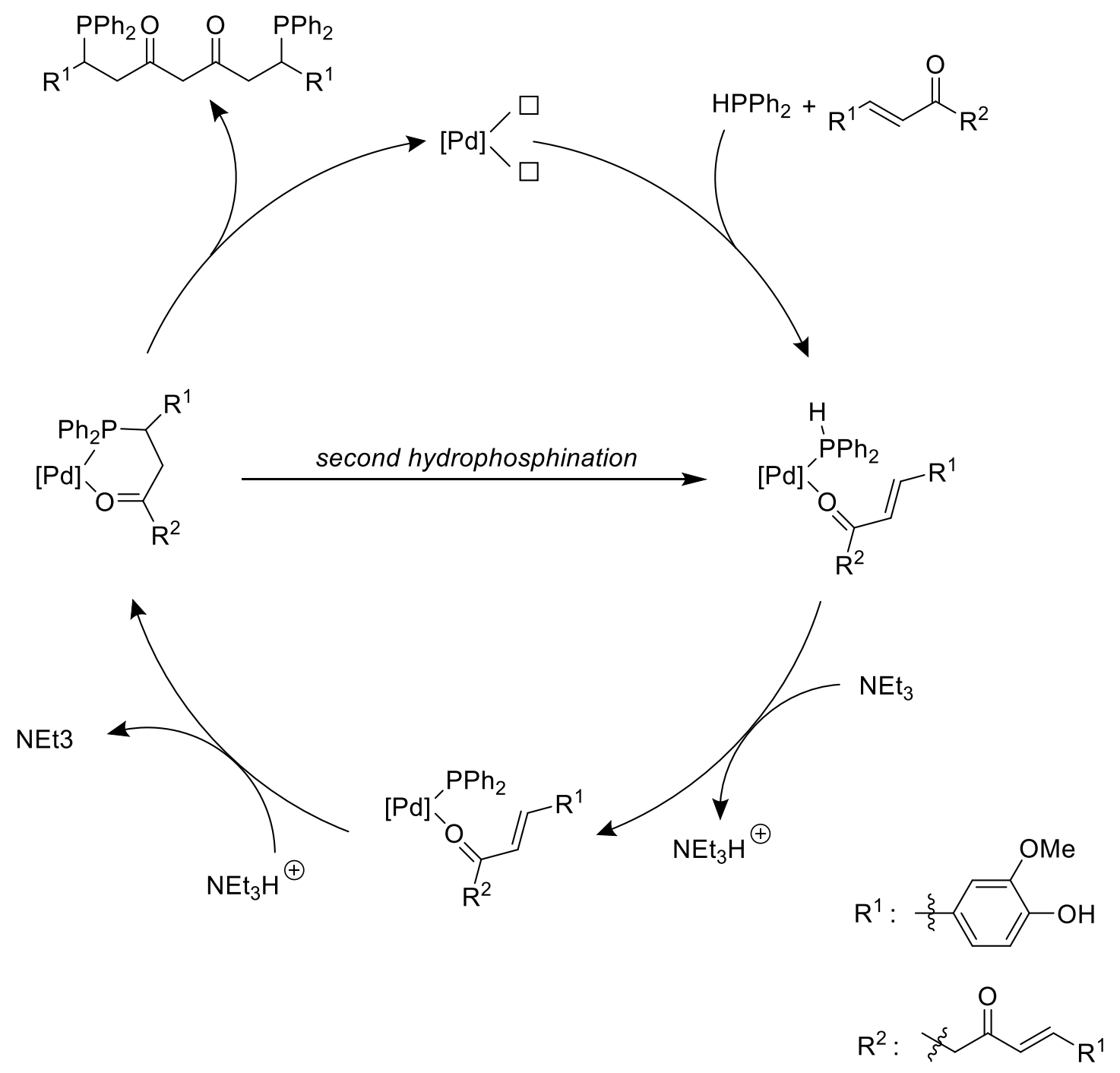

Figure S62. Proposed catalytic cycle to the asymmetric catalytic 1,2-dihydrophosphination of curcumin. 


\section{G X-RAY CRYSTALLOGRAPHIC DATA}

\section{G1 Sulfurized Mono-Hydrophosphinated Curcumin 2aa}

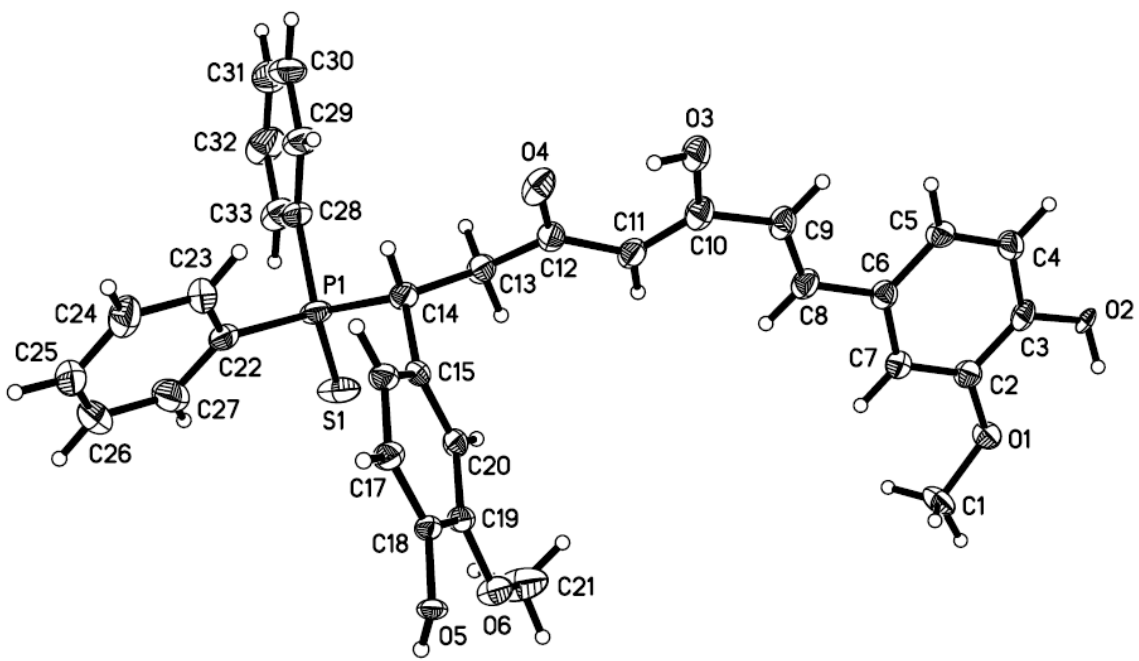

Figure S63. Molecular structure of sulfurized mono-hydrophosphinated curcumin 2aa with thermal ellipsoids shown at $50 \%$ probability.

\section{Depository Number}

CCDC Number

2090526

\section{Crystal Data}

Chemical Formula

Formula Weight (FW), g mol ${ }^{-1}$

$\mathrm{C}_{34} \mathrm{H}_{33} \mathrm{Cl}_{2} \mathrm{O}_{6} \mathrm{PS}$

Crystal System

671.53

monoclinic

Space Group

Temperature (K)

P $121 / \mathrm{c} 1$

$a, b, c(\AA)$

100(2)

$\alpha, \beta, \gamma\left({ }^{\circ}\right)$

$V\left(\AA^{3}\right)$

$Z$

$\mathrm{F}(000)$

12.7335(5), 8.8031(3), 28.6775(9)

90, 91.317(3), 90

3213.7(2)

4

1400

Radiation Type (Wavelength) Absorption Coefficient $\left(\mathrm{mm}^{-1}\right)$

1.54178

Crystal Size (mm)

3.265

$0.010 \times 0.020 \times 0.280$ 


\section{Data Collection}

Theta Range for Data Collection

3.08 to $68.28^{\circ}$

Index Ranges

Reflections Collected

$-15<=\mathrm{h}<=13,-10<=\mathrm{k}<=10,-30<=\mathrm{l}<=34$

Independent Reflections

23783

$T_{\min }, T_{\max }$

$5804[\mathrm{R}(\mathrm{int})=0.0909]$

$0.4620,0.9680$

\section{Refinement}

Refinement Method

Refinement Program

Function Minimized

No. of Reflections

No. of Restraints

Full-matrix least-squares on F2

No. of Parameters

SHELXL-2018/3 (Sheldrick, 2018)

Goodness-of-Fit on $\mathrm{F}^{2}$

$\Sigma \mathrm{w}\left(\mathrm{F}_{\mathrm{o}}^{2}-\mathrm{F}_{\mathrm{c}}^{2}\right)_{2}$

5804

1739

625

Final R Indices

1.027

4734 data; $\mathrm{I}>2 \sigma(\mathrm{I})$

$(\mathrm{R} 1=0.0884, \mathrm{wR} 2=0.2210)$

all data

$(\mathrm{R} 1=0.1001, \mathrm{wR} 2=0.2353)$

Weighting Scheme

$\left.w=1 /\left[\sigma^{2}\left(\mathrm{~F}_{\mathrm{o}}^{2}\right)+0.1627 \mathrm{P}\right)^{2}+2.3670 \mathrm{P}\right]$,

$r_{\max }, r_{\min }\left(\mathrm{e} \AA^{-3}\right)$
R.M.S. Deviation from Mean $\left(\mathrm{e} \AA^{-3}\right)$

where $\mathrm{P}=\left(\mathrm{F}_{\mathrm{o}}^{2}+2 \mathrm{~F}_{\mathrm{c}}^{2}\right) / 3$

$0.900,-1.299$

0.128 


\section{G2 Sulfurized Di-Hydrophosphinated Curcumin 3aa}

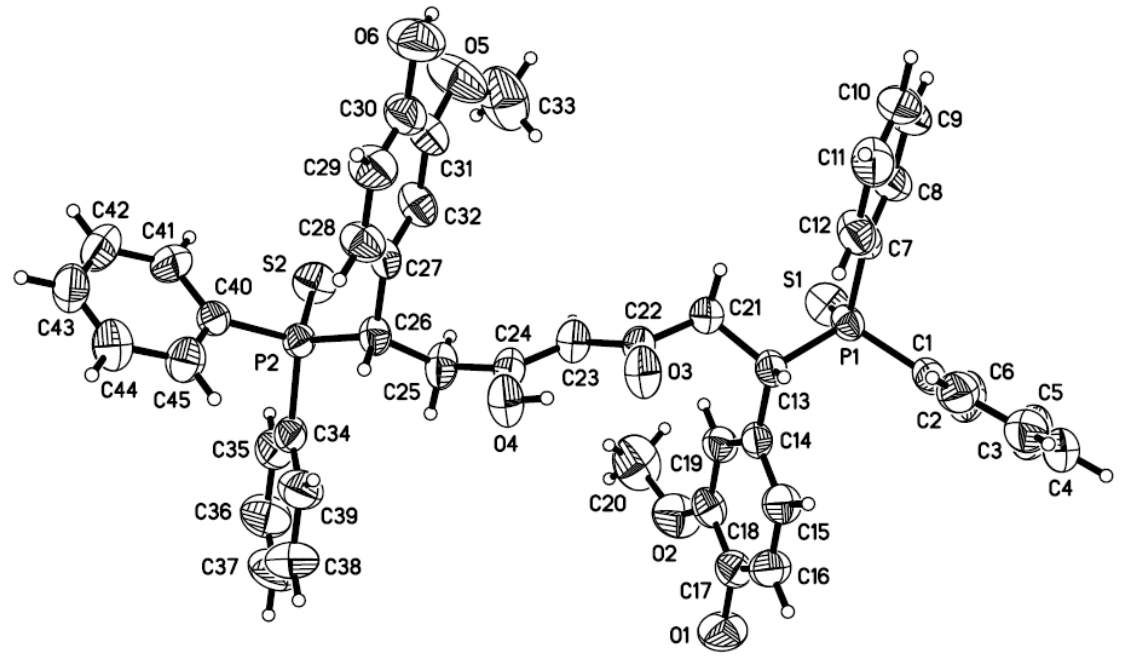

Figure S64. Molecular structure of sulfurized $d i$-hydrophosphinated curcumin 3aa with thermal ellipsoids shown at $50 \%$ probability. The structure presented in the figure has stereochemistry of $S$ and $S$ at $\mathrm{C} 21$ and $\mathrm{C} 26$.

\section{Depository Number}

CCDC Number

2093251

\section{Crystal Data}

Chemical Formula

Formula Weight (FW), $\mathrm{g} \mathrm{mol}^{-1}$

Crystal System

Space Group

Temperature $(\mathrm{K})$

$a, b, c(\AA)$

$\alpha, \beta, \gamma\left({ }^{\circ}\right)$

$V\left(\AA^{3}\right)$

$Z$

$\mathrm{F}(000)$

Radiation Type (Wavelength)

Absorption Coefficient $\left(\mathrm{mm}^{-1}\right)$

Crystal Size (mm)
$\mathrm{C}_{45} \mathrm{H}_{42} \mathrm{O}_{6} \mathrm{P}_{2} \mathrm{~S}_{2}$

804.84

monoclinic

P 1211

296(2)

6.8741(4), 35.809(2), 9.0649(5)

90, 111.159(4), 90

2080.9(2)

2

844

1.54178

2.268

$0.020 \times 0.200 \times 0.280$ 


\section{Data Collection}

Theta Range for Data Collection

Index Ranges

Reflections Collected

Independent Reflections

$T_{\min }, T_{\max }$
2.47 to $67.90^{\circ}$

$-8<=\mathrm{h}<=8,-42<=\mathrm{k}<=40,-10<=\mathrm{l}<=10$

27001

$7293[\mathrm{R}(\mathrm{int})=0.0550]$

$0.5690,0.9560$

\section{Refinement}

Refinement Method

Refinement Program

Function Minimized

No. of Reflections

No. of Restraints

No. of Parameters

Goodness-of-Fit on $\mathrm{F}^{2}$

$\Delta / \sigma_{\max }$

Final R Indices

Weighting Scheme

Absolute structure parameter

$r_{\max }, r_{\min }\left(\mathrm{e}^{-3}\right)$

R.M.S. Deviation from Mean $\left(\mathrm{e}^{-3}\right)$
Full-matrix least-squares on $\mathrm{F}^{2}$

SHELXL-2018/3 (Sheldrick, 2018)

$\Sigma \mathrm{w}\left(\mathrm{F}_{\mathrm{o}}^{2}-\mathrm{F}_{\mathrm{c}}{ }^{2}\right)^{2}$

7293

1

501

1.033

0.001

6742 data; $\mathrm{I}>2 \sigma(\mathrm{I})$

$(\mathrm{R} 1=0.0421, \mathrm{wR} 2=0.1024)$

all data

$(\mathrm{R} 1=0.0462, \mathrm{wR} 2=0.1051)$

$w=1 /\left[\sigma^{2}\left(\mathrm{~F}_{\mathrm{o}}{ }^{2}\right)+(0.0482 \mathrm{P})^{2}+0.3982 \mathrm{P}\right]$,

where $\mathrm{P}=\left(\mathrm{F}_{\mathrm{o}}^{2}+2 \mathrm{~F}_{\mathrm{c}}^{2}\right) / 3$

$0.030(10)$

$0.405,-0.203$

0.039 


\section{G3 Au(I) Di-Hydrophosphinated Curcumin Rac-4aa}

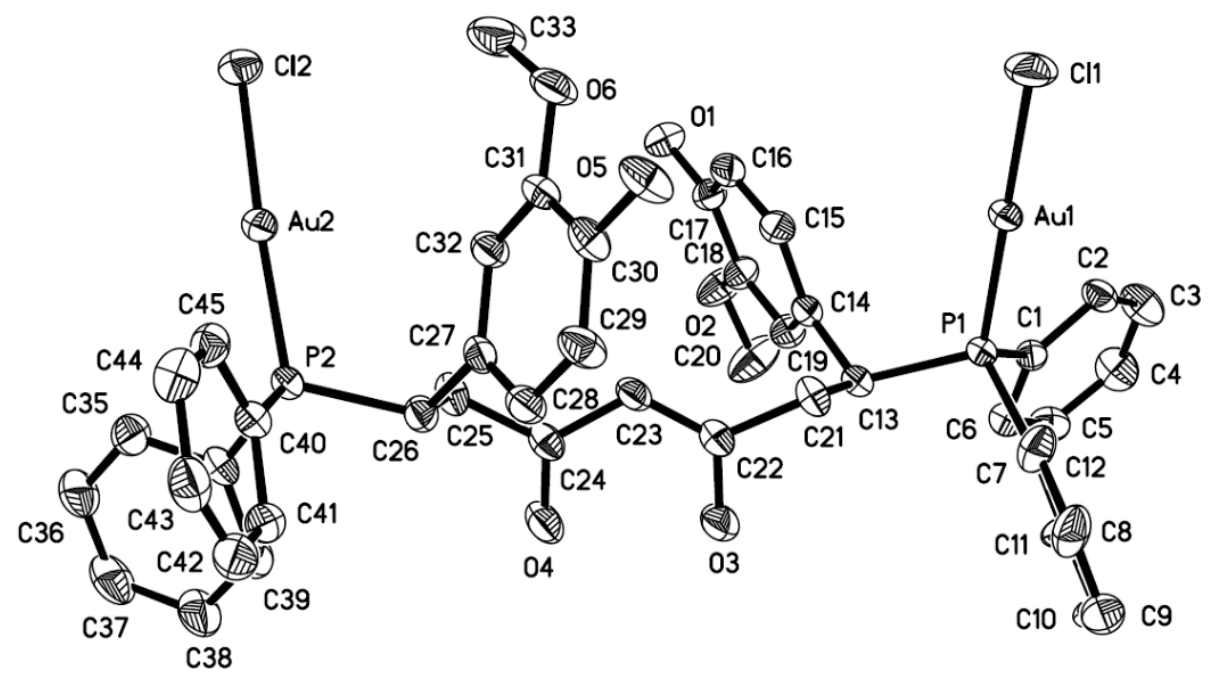

Figure S65. Molecular structure of $\mathrm{Au}(\mathrm{I}) d i$-hydrophosphinated curcumin rac-4aa with thermal ellipsoids shown at 50\% probability. Hydrogen atoms are omitted for clarity.

\section{Depository Number}

CCDC Number

2093252

\section{Crystal Data}

Chemical Formula

Formula Weight (FW), $\mathrm{g} \mathrm{mol}^{-1}$

$\mathrm{C}_{47.94} \mathrm{H}_{48.66} \mathrm{Au}_{2} \mathrm{Cl}_{3.22} \mathrm{O}_{6} \mathrm{P}_{2}$

Crystal System

1290.95

Space Group

Temperature $(\mathrm{K})$

monoclinic

P $121 / \mathrm{c} 1$

$a, b, c(\AA)$

100(2)

$\alpha, \beta, \gamma\left({ }^{\circ}\right)$

9.4719(2), 29.6113(5), 20.1946(3)

$V\left(\AA^{3}\right)$

90, 101.1373(8), 90

$Z$

$5557.41(17)$

$\mathrm{F}(000)$

4

Radiation Type (Wavelength) Absorption Coefficient $\left(\mathrm{mm}^{-1}\right)$

2508

0.71073

Crystal Size (mm)

5.526

$0.020 \times 0.140 \times 0.200$ 


\section{Data Collection}

Theta Range for Data Collection

2.30 to $34.34^{\circ}$

Index Ranges

Reflections Collected

$-15<=\mathrm{h}<=15,-43<=\mathrm{k}<=47,-32<=\mathrm{l}<=29$

Independent Reflections

78104

$T_{\min }, T_{\max }$

$23148[\mathrm{R}(\mathrm{int})=0.0518]$

$0.4040,0.8980$

\section{Refinement}

Refinement Method

Refinement Program

Function Minimized

No. of Reflections

No. of Restraints

Full-matrix least-squares on $\mathrm{F}^{2}$

No. of Parameters

SHELXL-2018/3 (Sheldrick, 2018)

Goodness-of-Fit on $\mathrm{F}^{2}$

Final R Indices

$\Sigma \mathrm{w}\left(\mathrm{F}_{\mathrm{o}}^{2}-\mathrm{F}_{\mathrm{c}}{ }^{2}\right)^{2}$

23148

430

660

1.047

15723 data; $\mathrm{I}>2 \sigma(\mathrm{I})$

$(\mathrm{R} 1=0.0498, \mathrm{wR} 2=0.1088)$

all data

$(\mathrm{R} 1=0.0889, \mathrm{wR} 2=0.1240)$

Weighting Scheme

$w=1 /\left[\sigma^{2}\left(\mathrm{~F}_{\mathrm{o}}{ }^{2}\right)+(0.0375 \mathrm{P})^{2}+22.2845 \mathrm{P}\right]$,

$\mathrm{r}_{\max }, \mathrm{r}_{\min }\left(\mathrm{e} \AA^{-3}\right)$

where $\mathrm{P}=\left(\mathrm{F}_{\mathrm{o}}^{2}+2 \mathrm{~F}_{\mathrm{c}}^{2}\right) / 3$

R.M.S. Deviation from Mean $\left(\mathrm{e} \AA^{-3}\right)$

2.550, - 2.173

0.181 


\section{G4 Au(I) Di-Hydrophosphinated Curcumin Meso-4aa'}

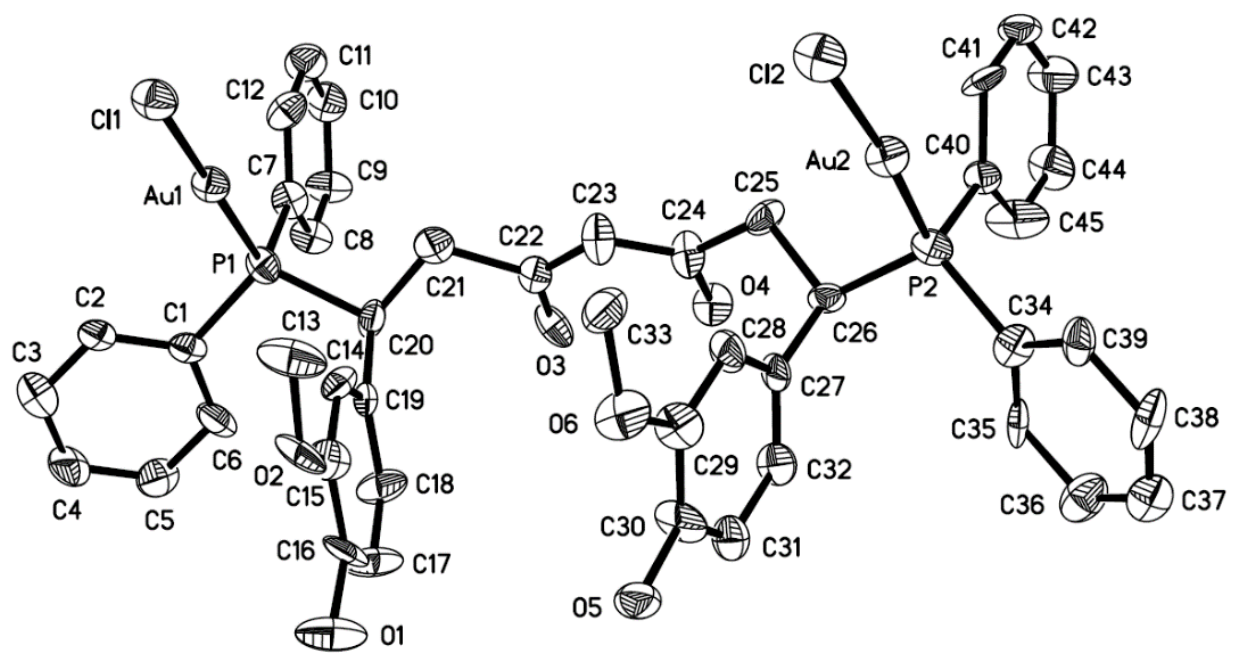

Figure S66. Molecular structure of $\mathrm{Au}(\mathrm{I})$ di-hydrophosphinated curcumin meso-4aa' with thermal ellipsoids shown at 50\% probability. Hydrogen atoms are omitted for clarity.

\section{Depository Number}

CCDC Number

2090525

\section{Crystal Data}

Chemical Formula

Formula Weight (FW), $\mathrm{g} \mathrm{mol}^{-1}$

$\mathrm{C}_{45.50} \mathrm{H}_{42} \mathrm{Au}_{2} \mathrm{Cl}_{3} \mathrm{O}_{6} \mathrm{P}_{2}$

Crystal System

1247.01

Space Group

Temperature $(\mathrm{K})$

orthorhombic

P 21212

$a, b, c(\AA)$

$100(2)$

$\alpha, \beta, \gamma\left({ }^{\circ}\right)$

27.9225(15), 44.567(2), 8.9967(6)

$V\left(\AA^{3}\right)$

90, 90, 90

$Z$

$11195.7(11)$

$\mathrm{F}(000)$

8

Radiation Type (Wavelength) Absorption Coefficient $\left(\mathrm{mm}^{-1}\right)$

4816

0.71073

Crystal Size (mm)

5.473

$0.010 \times 0.020 \times 0.200$ 


$\begin{array}{ll}\text { Data Collection } & \\ \text { Theta Range for Data Collection } & 2.23 \text { to } 26.39^{\circ} \\ \text { Index Ranges } & -34<=\mathrm{h}<=33,-51<=\mathrm{k}<=55,-11<=1<=11 \\ \text { Reflections Collected } & 71061 \\ \text { Independent Reflections } & 22707[\mathrm{R}(\mathrm{int})=0.1506] \\ T_{\min }, T_{\max } & 0.4070,0.9470\end{array}$

\section{Refinement}

Refinement Method

Full-matrix least-squares on $\mathrm{F}^{2}$

Refinement Program

SHELXL-2018/3 (Sheldrick, 2018)

Function Minimized

$\Sigma \mathrm{w}\left(\mathrm{F}_{\mathrm{o}}^{2}-\mathrm{F}_{\mathrm{c}}{ }^{2}\right)^{2}$

No. of Reflections

22707

No. of Restraints

869

No. of Parameters

911

Goodness-of-Fit on $\mathrm{F}^{2}$

0.953

$\Delta / \sigma_{\max }$

0.001

Final R Indices

13380 data; $\mathrm{I}>2 \sigma(\mathrm{I})$

$(\mathrm{R} 1=0.0619, \mathrm{wR} 2=0.1084)$

all data

$(\mathrm{R} 1=0.1312, \mathrm{wR} 2=0.1322)$

Weighting Scheme

$w=1 /\left[\sigma^{2}\left(\mathrm{~F}_{\mathrm{o}}{ }^{2}\right)+(0.0280 \mathrm{P})^{2}\right]$,

$\mathrm{r}_{\max }, \mathrm{r}_{\min }\left(\mathrm{e} \AA^{-3}\right)$

where $\mathrm{P}=\left(\mathrm{F}_{\mathrm{o}}^{2}+2 \mathrm{~F}_{\mathrm{c}}^{2}\right) / 3$

$1.108,-1.097$

R.M.S. Deviation from Mean $\left(\mathrm{e} \AA^{-3}\right)$

0.178 